This document was prepared in conjunction with work accomplished under Contract No. DE-AC09-96SR18500 with the U. S. Department of Energy.

\title{
DISCLAIMER
}

This report was prepared as an account of work sponsored by an agency of the United States Government. Neither the United States Government nor any agency thereof, nor any of their employees, nor any of their contractors, subcontractors or their employees, makes any warranty, express or implied, or assumes any legal liability or responsibility for the accuracy, completeness, or any third party's use or the results of such use of any information, apparatus, product, or process disclosed, or represents that its use would not infringe privately owned rights. Reference herein to any specific commercial product, process, or service by trade name, trademark, manufacturer, or otherwise, does not necessarily constitute or imply its endorsement, recommendation, or favoring by the United States Government or any agency thereof or its contractors or subcontractors. The views and opinions of authors expressed herein do not necessarily state or reflect those of the United States Government or any agency thereof. 


\section{CHARACTERIZATION AND SETTLING TESTS WITH TANK 51H SLURRY SAMPLES HTF-076-081}

January 9, 2006

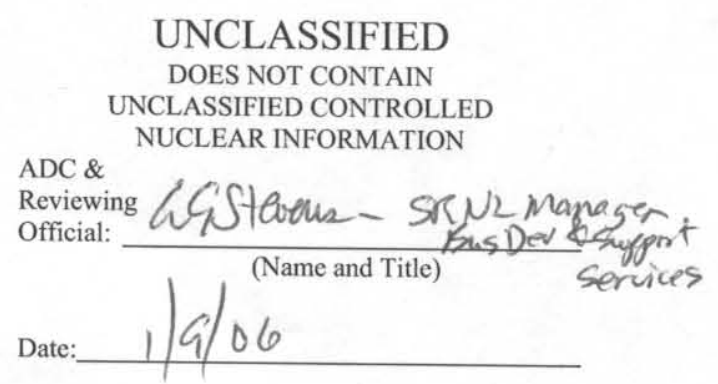

Waste Processing Technology Section Savannah River National Laboratory Aiken, SC 29808

Prepared for the U.S. Department of Energy Under Contract Number DEAC09-96SR18500

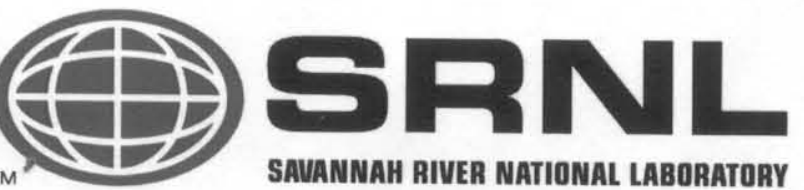




\section{DISCLAIMER}

This report was prepared for the United States Department of Energy under Contract No. DE-AC09-96SR18500 and is an account of work performed under that contract. Neither the United States Department of Energy, nor WSRC, nor any of their employees makes any warranty, expressed or implied, or assumes any legal liability or responsibility for the accuracy, completeness, or usefulness, of any information, apparatus, or product or process disclosed herein or represents that its use will not infringe privately owned rights. Reference herein to any specific commercial product, process, or service by trade name, trademark, name, manufacturer or otherwise does not necessarily constitute or imply endorsement, recommendation, or favoring of same by Westinghouse Savannah River Company or by the United States Government or any agency thereof. The views and opinions of the authors expressed herein do not necessarily state or reflect those of the United States Government or any agency thereof.

\section{Printed in the United States of America}

Prepared For

\section{U.S. Department of Energy}


Key Words:

Sludge Analysis

Supernate Analysis

Sludge Settling

\section{Retention: Permanent}

Key References:

TTR: HLW/DWPF/TTR-2005-0018

Task Plan: WSRC-RP-2005-01683

Analytical Plan: SRNL-ITS-2005-00179

\section{CHARACTERIZATION AND SETTLING TESTS WITH TANK 51H SLURRY SAMPLES HTF-076-081}

M. S. Hay, SRNL/CS\&T

T. L. Fellinger, SRNL/PS\&E

Issue Date: January 9, 2006

Waste Processing Technology Section Savannah River National Laboratory Aiken, SC 29808

Prepared for the U.S. Department of Energy Under Contract Number DEAC09-96SR18500

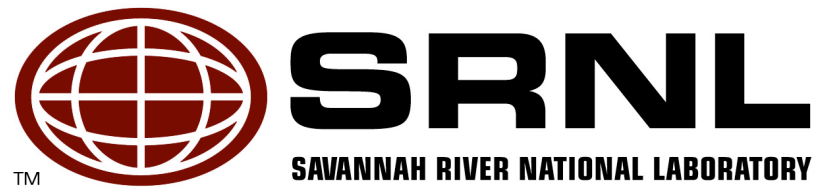


WSRC-TR-2005-00525, REV. 0

REVIEWS AND APPROVALS
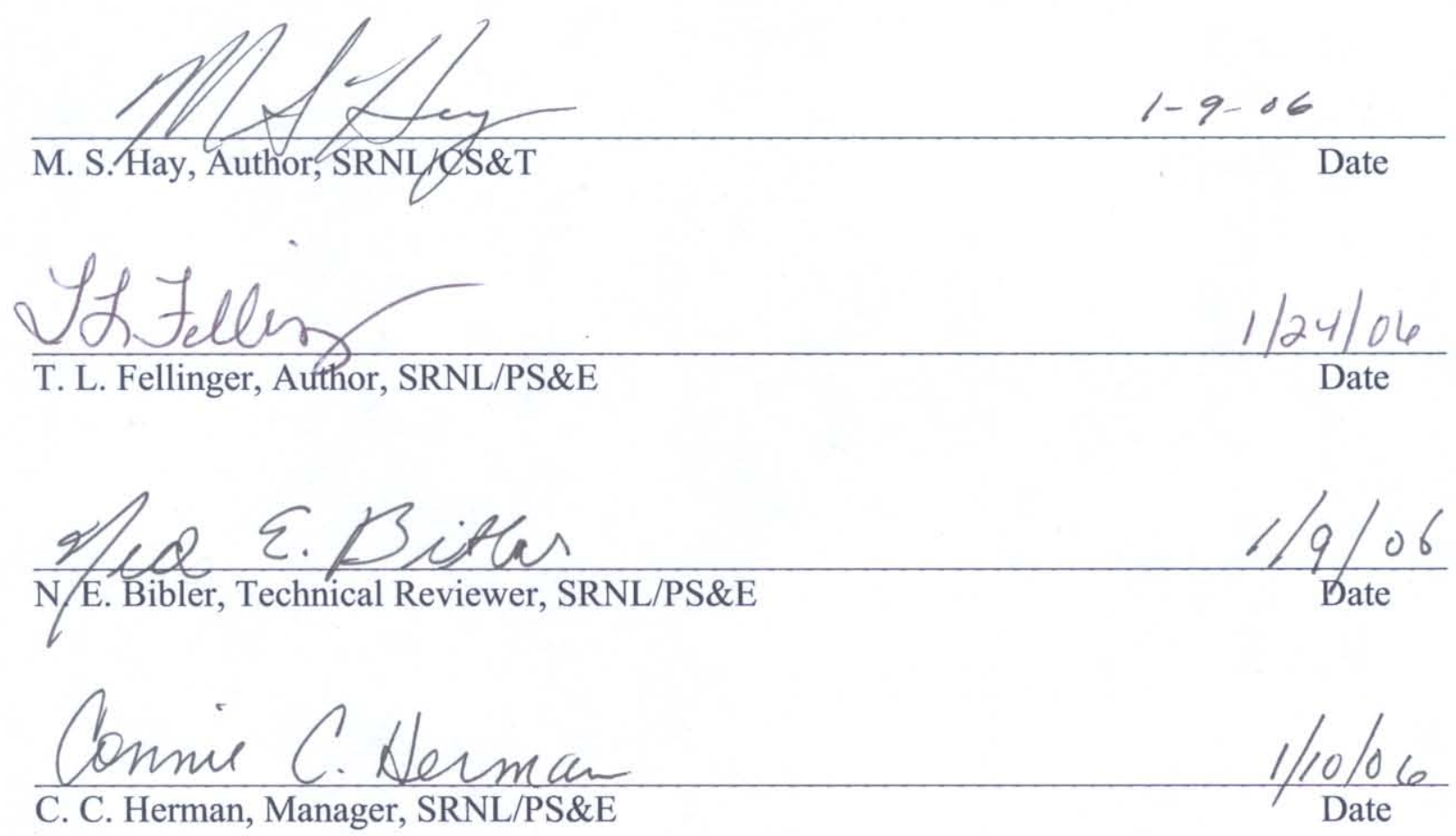

C. C. Herman, Manager, SRNL/PS\&E

Date

J. E. Occhipinti, Manager, CBU/WSE

$\frac{1 / 23 / 06}{\text { bate }}$

Maria A Rios-Arnstrong

M. A. Rios-Armstrong, CBU/PIT-Sludge Disposition Planning

$\frac{1 / 25 / 06}{\text { Date }}$

- ii - 


\section{TABLE OF CONTENTS}

List of Tables $\quad$ iv

List of Figures $\quad$ iv

List of Acronyms $\quad$ v

SUMMARY vi

$\begin{array}{ll}\text { 1.0 INTRODUCTION } & 1\end{array}$

2.0 SAMPLE RECEIPT, COMPOSITING, AND PREPARATION 2

2.1 SAMPLE RECEIPT

2

2.2 SAMPLE PREPARATION FOR ANALYSIS

2.3 WeIGHT PERCENT SOLIDS AND DENSITY MEASUREMENTS

3.0 RESULTS OF THE SAMPLE CHARACTERIZATION

3.1 GENERAL INFORMATION

3.2 RESUlts OF THE WeIGHT PERCENT SOlids, DENSITY, AND PH MEASUREMENTS OF THE TANK 51H

SAMPLE

3.3 RESUlTS OF THE ANALYSIS OF THE TANK 51H FILTERED SUPERNATE SAMPLE

3.4 RESULTS OF THE ANALYSIS OF THE TANK 51H TOTAL DRIED SOLIDS

\subsection{SLUDGE SETTLING TESTS}

4.1 INITIAL SETTLING TEST OF TANK 51H SLUDGE

4.2 BlENDING TANK 51H SLUdGE With SB3 SLudGE

4.3 HeAt TREATMENT OF TANK 51H AND TANK 51/SB3 Blend SludGE

4.4 CONCLUSIONS FROM THE SETTLING TESTS

\subsection{GENERAL DESCRIPTION OF ANALYTICAL PROCEDURES}

5.1 INDUCTIVELy COUPLED PlaSMA-ATOMIC EMISSION SPECTROSCOPY

5.2 ATOMIC ABSORPTION SPECTROSCOPY

5.3 ION CHROMATOGRAPHY

5.5 INDUCTIVELY COUPLED PLASMA-MASS SPECTROMETRY

5.6 GAMMA SPECTROMETRY

5.7 $\mathrm{SR}^{90}$ ANALYSIS

5.8 AlPHA SPECTROSCOPY FOR PLUTONIUM ISOTOPICS 


\section{List of Tables}

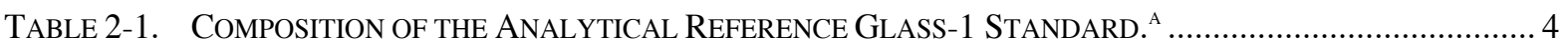

TABLE 3-1. ABBREVIATIONS FOR ANALYTICAL METHODS USED IN DATA TABLES ........................................... 6

TABle 3-2. Weight Percent SoldDS, Density, AND PH RESUlTS From TANK 51 SAMPLE HTF-076-81 ........ 9

TABle 3-3. COMPOSITION OF THE Filtered SuPERnATE FROM TANK 51 SAMPLE HTF-076-81 ..................... 10

TABle 3-4. COMParison of All Dissolution Data For the Total Dried Solids From Tank 51 SAmple

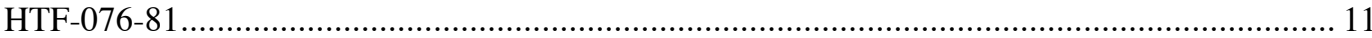

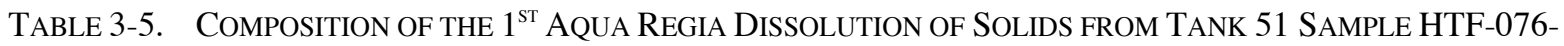

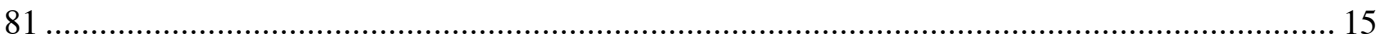

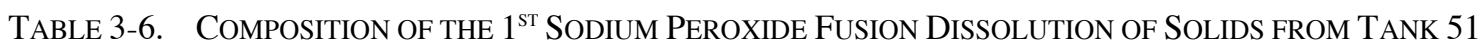

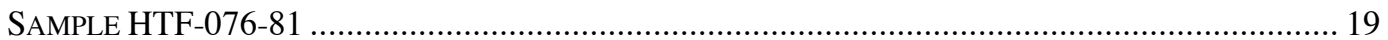

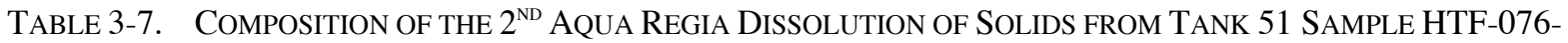

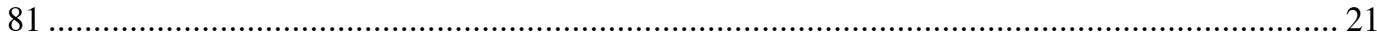

TABle 3-8. COMPOSITION OF THE $2^{\text {ND }}$ SODIUM PEROXIDE FusION DISSOLUTION OF SOLIDS FROM TANK 51

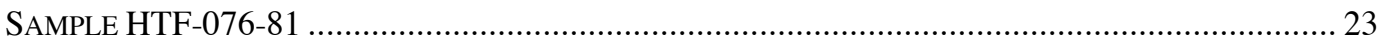

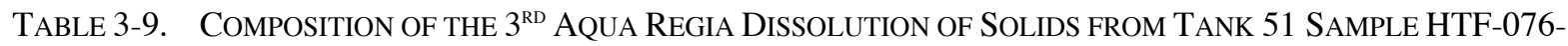

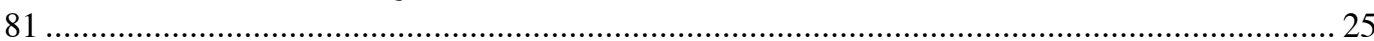

TABle 3-10. COMPOSITION OF THE $3^{\text {RD }}$ SODIUM PEROXIDE Fusion DisSOlution OF SOLIDS FROM TANK 51 SAMPLE HTF-076-81

TABLE 4-1. TARGET COMPOSITION FOR TANK 51H AFTER ADDITION OF TANK 5F AND 6F COMPARED WITH THE CALCUlated COMPOSITION OF THE TANK 51H/SB3 BLEND

\section{List of Figures}

FIGURE 3-1. XRD OF UNDISSOLVED SOLIDS FILTERED FROM AQUA-REGIA DISSOLUTIONS............................... 31

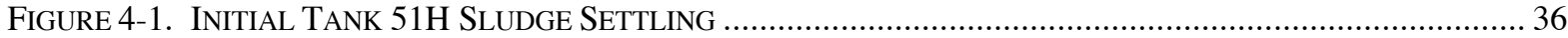

FiguRE 4-2. TANK 51H SLUdGe SETTLING VERSUS A BLEND OF TANK 51H AND SB3 SLUDGE ............................ 37

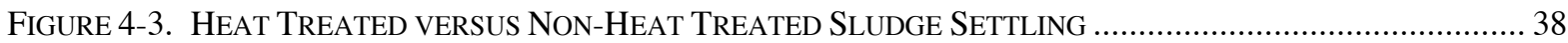

FiguRE 4-4. All SLUdGE SETTLING DATA FOR TANK 51H SLUDGE ........................................................... 39 


\section{List of Acronyms}

$\begin{array}{ll}\text { DWPF } & \text { Defense Waste Processing Facility } \\ \text { SRAT } & \text { Sludge Receipt and Adjustment Tank } \\ \text { CPC } & \text { Chemical Processing Cell } \\ \text { SB3 } & \text { Sludge Batch 3 } \\ \text { SB4 } & \text { Sludge Batch 4 } \\ \text { SRNL } & \text { Savannah River National Laboratory } \\ \text { TTR } & \text { Technical Task Request } \\ \text { ADS } & \text { Analytical Development Section } \\ \text { ICP-ES } & \text { Inductively Coupled Plasma-Emission Spectroscopy } \\ \text { ICP-MS } & \text { Inductively Coupled Plasma-Mass Spectrometry } \\ \text { IC } & \text { Ion Chromatography } \\ \text { ARG1 } & \text { Analytical Reference Glass 1 } \\ \text { \%RSD } & \text { Percent Relative Standard Deviation } \\ \text { DL } & \text { Detection Limit } \\ \text { T } & \text { Titration } \\ \text { CV } & \text { Atomic Absorption Cold Vapor Method } \\ \text { GS } & \text { Gamma Scan } \\ \text { IE } & \text { Inductively Coupled Plasma-Emission Spectroscopy } \\ \text { IM } & \text { Inductively Coupled Plasma-Mass Spectrometry } \\ \text { BS } & \text { Beta Liquid Scintillation } \\ \text { AQ } & \text { Aqua-Regia Dissolution } \\ \text { PF } & \text { Sodium Peroxide Fusion Dissolution } \\ \text { TTA } & \text { Thenoyltrifluoroacetone Separation/Alpha Counting } \\ \text { XRD } & \text { X-ray Diffraction }\end{array}$


WSRC-TR-2005-00525, REV. 0

\section{SUMMARY}

Sludge Batch 4 (SB4) is the next sludge batch being prepared for feed to the Defense Waste Processing Facility (DWPF). SB4 includes sludge from Tanks 5F, 6F, and $11 \mathrm{H}$ and heels from Tanks $7 \mathrm{~F}$ and $51 \mathrm{H}$. In preparation of SB4, sludge was transferred from Tank $11 \mathrm{H}$ to Tank $51 \mathrm{H}$. The sludge currently in Tank $51 \mathrm{H}$ has been found to settle at slower rates than previous sludge batches. The slow sludge settling in Tank $51 \mathrm{H}$ impacts the ability to wash SB4 to the desired final weight percent insoluble solids and sodium endpoint. This could impact the ability to have SB4 ready on time to support DWPF and result in increased recycle back to the Tank Farm, reduced waste loading at DWPF, and lengthened cycle time in the DWPF Chemical Processing Cell (CPC) Sludge Receipt and Adjustment Tank (SRAT).

The Savannah River National Laboratory (SRNL) was requested to characterize and investigate the slower settling rate with six slurry dip samples of Tank $51 \mathrm{H}$ sludge. The filtered supernate and the total dried solids of the sludge were analyzed and summaries of the results published in the references listed below. ${ }^{*}$ The sludge composition was found to be consistent with H-Area high aluminum sludge. Difficulties were encountered with dissolving all of the material in the dried sludge solids. An analysis of the undissolved material from the digestions found the main constituent was Boehmite $(\mathrm{AlO}(\mathrm{OH}))$. This report provides all of the compositional data and an analysis of the data with recommended values to use for the composition of the Tank $51 \mathrm{H}$ composite sample. Tables 3-2 through 3-4 provide the composition of the Tank $51 \mathrm{H}$ composite sample.

Settling tests conducted with the Tank $51 \mathrm{H}$ sludge showed a much slower settling rate than with the sludge in Sludge Batch 3 (SB3). A mixture of Tank 51H and sludge from SB3 was prepared to mimic the projected final composition of Sludge Batch 4 (SB4). The mixture showed minimal improvement in the settling rate versus Tank $51 \mathrm{H}$ sludge alone. An attempt to increase the settling rate of Tank $51 \mathrm{H}$ sludge by adjusting the sludge to a $1.0 \mathrm{M}$ free hydroxide concentration and heating to $60-65^{\circ} \mathrm{C}$ for four days also showed no improvement in the settling rate. Limited flocculent addition testing has been performed with simulants to try and improve the settling rate and has been documented in a separate memorandum. Testing of additional additives with sludge simulants is being pursued and any found to be successful will be tested with the Tank $51 \mathrm{H}$ sludge sample.

\footnotetext{
* Summary's of the results of the analysis were published in; T. L. Fellinger, Corrosion Results for Tank $51 H$ Samples HTF-076 - HTF-081 (U), SRNL-ITS-2005-00203, August 19, 2005 and T. L. Fellinger, M. S. Hay, Chemical and Radionuclide Analysis Results for the Tank 51 H Samples HTF-076-HTF-081 (U), SRNL-ITS2005-0268, November 9, 2005.

** C. C. Herman, F. F. Fondeur, D. R. Click, M. E. Stone, Initial Results for SRNL Characterization and Settling Testing of Tank 51-Sludge Batch 4, SRNL-ITS-2005-00272, December 5, 2005.
} 
WSRC-TR-2005-00525, REV. 0

\subsection{INTRODUCTION}

DWPF is currently processing and immobilizing radioactive sludge slurry into a durable borosilicate glass. DWPF has already processed three sludge batches (Sludge Batch 1A, Sludge Batch 1B, and Sludge Batch 2) and is currently processing the fourth sludge batch designated Sludge Batch 3. Preparations of the fifth sludge batch, Sludge Batch 4, are currently ongoing in Tank $51 \mathrm{H}$.

In preparation of SB4, sludge was transferred from Tank $11 \mathrm{H}$ to Tank $51 \mathrm{H}$. The sludge transfer required the use of hundreds of thousands of gallons of inhibited water and high shear pumps in order to break up and suspend the solids in Tank $11 \mathrm{H}$. After the transfers of Tank $11 \mathrm{H}$ material to Tank $51 \mathrm{H}$ were complete, the sludge was allowed to settle. Sludge interface level measurements in the tank indicated the sludge was settling more slowly than expected. Potential impacts to Tank Farm operations and DWPF due to the slower than expected sludge settling rate may include:

- Inability to wash the sludge to the desired sodium molarity could impact waste loading and glass properties. This, coupled with the high aluminum concentration in SB4, could also lead to nepheline formation and slower melt rate.

- $\quad$ Reduced washing to meet DWPF feed schedule requirements could impact glass formulation and lead to reduced waste loadings.

- SB4 feed preparation delays could occur as a result of the slower settling rates due to a higher sludge layer in the Tank $51 \mathrm{H}$. This could result in a feed break and a delay in canister production in DWPF.

- Inability to obtain the target final SB4 weight percent insoluble solids in Tank $51 \mathrm{H}$. Depending on final weight percent insoluble solids achieved, this could potentially increase DWPF recycle volume to the Tank Farm. Lower final weight percent insoluble solids could also lengthen the DWPF CPC SRAT cycle.

Upon discovery of the reduced settling rate in Tank $51 \mathrm{H}$, personnel from DWPF, Liquid Waste Disposition, Closure Business Unit - Planning, Integration, and Technology, and SRNL held a brain storming meeting to discuss possible causes for the slower settling rate. The recommendations and path forward have been summarized in memo SRNL-ITS-200500125.

DWPF - Engineering issued a Technical Task Request (TTR) for SRNL to investigate the slower settling rate in Tank $51 \mathrm{H}^{2}$ A task plan ${ }^{3}$ and analytical study plan ${ }^{4}$ were issued by SRNL describing the steps needed to meet the requirements of the TTR. In order to perform the tasks requested, sludge slurry samples from Tank $51 \mathrm{H}$ were obtained and sent to the SRNL Shielded Cells Facility for characterization and testing. 
WSRC-TR-2005-00525, REV. 0

\subsection{SAMPLE RECEIPT, COMPOSITING, AND PREPARATION}

\subsection{SAMPLE RECEIPT}

Six $\sim 80 \mathrm{~mL}$ slurry dip samples from Tank 51H, labeled HTF- 076 through HTF-081, were received at SRNL on July 20, 2005. The sample numbers were entered in the SRNL Shielded Cells logbook as required by sample tracking procedures. The samples were composited into a $1 \mathrm{~L}$ calibrated poly bottle. After the solids had settled in the composite sample, a portion of the supernate was decanted and used to rinse any remaining solids from the dip sample vials into the compositing vessel. The total volume of the composite sample was $\sim 480 \mathrm{~mL}$.

\subsection{SAMPLE PREPARATION FOR ANALYSIS}

A portion of the composite slurry sample was filtered through a $0.45 \mu$ nylon disposable filter to obtain the clear supernate phase. Portions of the filtered supernate were diluted by a factor of $\sim 5$ with de-ionized distilled water and submitted to the Analytical Development Section (ADS) for Inductively Couple Plasma-Emission Spectroscopy (ICP-ES), Ion Chromatography (IC), and Gamma Scan. Samples of the undiluted supernate were also removed from the Shielded Cells and submitted to ADS to perform the Free Hydroxide and Total Base analyses. All sample preparations of the filtered supernate samples were conducted in triplicate. Additionally, a water blank and an ICP-ES standard solution were submitted with each set of filtered supernate samples.

Three aliquots of the mixed sludge slurry composite sample were dried overnight in a drying oven at $115^{\circ} \mathrm{C}$. Weighted portions of the dried solids from the three aliquots were prepared for analysis by aqua-regia digestion and fusion with sodium peroxide followed by uptake in hydrochloric acid. A digestion of a glass standard containing many of the elements found in tank samples was prepared concurrently with the sample digestions. Table 2-1 lists the composition of the Analytical Reference Glass-1 (ARG-1) glass standard. ${ }^{5}$ A reagent blank was also prepared consisting of the digestion reagents and incorporated any manipulations and dilutions conducted on the sample.

\subsection{WEIGHT PERCENT SOLIDS AND DENSITY MEASUREMENTS}

The density of the filtered supernate and the well-mixed slurry sample were measured in the Shielded Cells using calibrated plastic tubes with a nominal volume of $\sim 8.25 \mathrm{~mL}$. The density measurements were conducted in triplicate on each phase of the sample.

The weight percent total solids in the slurry sample were measured in the Shielded Cells using a conventional drying oven at $115^{\circ} \mathrm{C}$. The sample was dried until repeated weights indicated no further loss of water. The weight percent dissolved solids in a sample of the filtered supernate were measured in the same manner. All weight percent solids measurements were made in triplicate. The weight percent insoluble solids and weight percent soluble solids in the slurry sample were calculated using the equations shown below. 
$\mathrm{Wis}=(\mathrm{Wts}-\mathrm{Wds}) /(1-\mathrm{Wds}) \quad$ and $\quad$ Wss $=\mathrm{Wts}-\mathrm{Wis}$

where:

Wis $=$ weight fraction of insoluble solids in the slurry

Wss $=$ weight fraction of soluble solids in the slurry

Wts $=$ weight fraction of total solids in the slurry

$\mathrm{Wds}=$ weight fraction of dissolved solids in the filtered supernate

Thus:

$\mathrm{Wt} \%$ dissolved solids $=\quad(\mathrm{wt}$ dissolved solids $/ \mathrm{wt}$ of supernate $) \times 100$

$\mathrm{Wt} \%$ total solids $=$ (wt total solids/wt of total slurry) x 100

$\mathrm{Wt} \%$ insoluble solids $=$ (wt insoluble solids/wt of total slurry) x 100

$\mathrm{Wt} \%$ soluble solids $=$ (wt of dissolved solids/wt of total slurry) x 100 
Table 2-1. Composition of the Analytical Reference Glass-1 Standard. ${ }^{\text {a }}$

\begin{tabular}{|l|c|c|}
\hline Element & Wt\% in Glass & $\mathbf{m g} / \mathbf{K g}$ in Glass \\
\hline $\mathrm{Al}$ & $2.50 \%$ & $2.50 \mathrm{E}+04$ \\
\hline $\mathrm{B}$ & $2.69 \%$ & $2.69 \mathrm{E}+04$ \\
\hline $\mathrm{Ba}$ & $0.079 \%$ & $7.90 \mathrm{E}+02$ \\
\hline $\mathrm{Ca}$ & $1.02 \%$ & $1.02 \mathrm{E}+04$ \\
\hline $\mathrm{Cr}$ & $0.064 \%$ & $6.40 \mathrm{E}+02$ \\
\hline $\mathrm{Cu}$ & $0.003 \%$ & $3.0 \mathrm{E}+01$ \\
\hline $\mathrm{Fe}$ & $9.79 \%$ & $9.79 \mathrm{E}+04$ \\
\hline $\mathrm{K}$ & $2.26 \%$ & $2.26 \mathrm{E}+04$ \\
\hline $\mathrm{Li}$ & $1.49 \%$ & $1.49 \mathrm{E}+04$ \\
\hline $\mathrm{Mg}$ & $0.52 \%$ & $5.2 \mathrm{E}+03$ \\
\hline $\mathrm{Mn}$ & $1.46 \%$ & $1.46 \mathrm{E}+04$ \\
\hline $\mathrm{Na}$ & $8.52 \%$ & $8.52 \mathrm{E}+04$ \\
\hline $\mathrm{Ni}$ & $0.83 \%$ & $8.3 \mathrm{E}+03$ \\
\hline $\mathrm{P}$ & $0.11 \%$ & $1.1 \mathrm{E}+03$ \\
\hline $\mathrm{Si}$ & $22.4 \%$ & $2.24 \mathrm{E}+05$ \\
\hline $\mathrm{Sr}$ & $0.003 \%$ & $3.0 \mathrm{E}+01$ \\
\hline $\mathrm{Ti}$ & $0.69 \%$ & $6.9 \mathrm{E}+03$ \\
\hline $\mathrm{Zn}$ & $0.016 \%$ & $1.6 \mathrm{E}+02$ \\
\hline $\mathrm{Zr}$ & $0.096 \%$ & $9.6 \mathrm{E}+02$ \\
\hline
\end{tabular}

a The table was obtained from: C. J. Coleman, R. A. Dewberry, M. F. Bryant, J. J. Gemmill, SRL's Performance in Round Robin \#6 - Analysis of Simulated Defense Waste Glass (U), WSRC-TR-91-187, Rev. 0, May 31, 1991. 
WSRC-TR-2005-00525, REV. 0

\subsection{RESULTS OF THE SAMPLE CHARACTERIZATION}

\subsection{GENERAL INFORMATION}

Tables 3-2 through 3-10 provide the chemical composition of the Tank 51H composite slurry sample received at SRNL. Analytical results for samples use units of molarity for the supernate sample and $\mathrm{mg} / \mathrm{Kg}$ of dried solids for the solids dissolution samples. (Note: To convert values in $\mathrm{mg} / \mathrm{Kg}$ to a weight percent solids basis, divide by $1 \mathrm{E}+04$.) Table 3-4 presents the recommended values for the composition of the Tank $51 \mathrm{H}$ composite sample. The recommended values for the composition of the Tank $51 \mathrm{H}$ composite sample were previously published. ${ }^{6}$

The tables include the results of all replicates, blanks, and glass standards. The analytical results in tables for the solids dissolution samples include a data quality flag in the last column to indicate potential problems with the data quality. The presence of a data quality flag for an analyte does not mean the data should not be used, however, the end user of the data should be aware the result may have a higher than normal uncertainty. For waste tank sample characterization, an uncertainty of approximately $+/-15 \%$ has been found to be the normal range for the combined sampling and analytical uncertainty. ${ }^{7}$ For the Tank $51 \mathrm{H}$ composite sample, the percent relative standard deviation (\%RSD) presented in the tables only includes the uncertainty associated with sub-sampling and the analytical method. It should be noted that the Tank $51 \mathrm{H}$ composite sample represents a small amount of material from a large tank. The following defines the data quality flags used in the tables:

$\mathrm{U}_{\mathrm{R}} \quad$ The \%RSD for the three sample replicates was higher than $15 \%$. This flag indicates higher than normal uncertainty in the sample replicates.

$\mathrm{U}_{\mathrm{B}} \quad$ The analyte concentration in the blank exceeds $5 \%$ of the concentration measured in the sample. The presence of contaminants in the blank indicates the potential contamination of the samples during sample preparation. However, the contamination level in the samples would probably be random in nature and, therefore, be reflected in a higher $\%$ RSD also.

$\mathrm{U}_{\mathrm{E}} \quad$ The analyte concentration was measured at less than ten times the detection limit (DL) for the sample. Analyte concentrations less than 10X the DL usually have a higher associated uncertainty. Again, the higher uncertainty of results with this flag would generally be reflected by a higher \%RSD.

$\mathrm{U}_{\mathrm{G}} \quad$ The glass standard, prepared concurrently with the solids dissolutions of the sample, indicates a greater than $15 \%$ difference in the measured versus the known standard concentration. This flag can indicate potential problems during the sample preparation or with the analytical instrument measuring the analyte.

The data tables also indicate the analytical method used to measure each analyte. Table 3-1 shows the abbreviations used for each analytical method: 
WSRC-TR-2005-00525, REV. 0

Table 3-1. Abbreviations for Analytical Methods used in Data Tables

\begin{tabular}{|l|c|}
\hline Analytical Method & $\begin{array}{c}\text { Abbreviation } \\
\text { in Tables }\end{array}$ \\
\hline Inductively Coupled Plasma-Emission Spectroscopy & IE \\
\hline Ion Chromatography & IC \\
\hline Titration & T \\
\hline Atomic Absorption Cold Vapor Method & CV \\
\hline Gamma Scan & GS \\
\hline Inductively Coupled Plasma-Mass Spectrometry & IM \\
\hline Beta Liquid Scintillation & BS \\
\hline Thenoyltrifluoroacetone Separation/Alpha Counting & TTA \\
\hline
\end{tabular}

\subsection{RESULTS OF THE WEIGHT PERCENT SOLIDS, DENSITY, AND PH MEASUREMENTS OF THE TANK 51H SAMPLE}

Table 3-2 shows the results of the weight percent solids, density, and $\mathrm{pH}$ measurements made on the Tank 51H composite sample. All measurements were made in triplicate unless otherwise noted in the table. The replicate measurements from each analysis show good agreement as indicated by the low \%RSD. As stated in section 2.3, the weight percent insoluble solids and weight percent soluble solids of the slurry were calculated from the measurements of the weight percent total solids and weight percent dissolved solids. Only a single $\mathrm{pH}$ measurement was made on the slurry and filtered supernate of the sample.

\subsection{RESULTS OF THE ANALYSIS OF THE TANK 51H FILTERED SUPERNATE SAMPLE}

Table 3-3 provides the results of the analysis of the filtered supernate from the Tank $51 \mathrm{H}$ composite sample. All analyses were conducted in triplicate unless otherwise noted in the table. These results were reported previously in a memorandum. ${ }^{8}$ A comparison of the sum of the cations versus the sum of the anion species in the analysis show good agreement with a difference of $2.3 \%$. The sulfur by ICP-ES and the sulfate by IC show excellent agreement with a difference of less than $1 \%$.

\subsection{RESULTS OF THE ANALYSIS OF THE TANK 51H TOTAL DRIED SOLIDS}

Tables 3-4 through 3-10 show the results of the analysis of the aqua regia (AQ) and sodium peroxide fusion (PF) dissolutions of the total dried solids obtained from the Tank $51 \mathrm{H}$ composite sample (HTF-076-081). Table 3-5 and 3-6 show the results of the first set of aquaregia and sodium peroxide fusion dissolutions conducted on the total dried solids of the Tank $51 \mathrm{H}$ composite sample. When the results from this analysis were received, the aluminum in the aqua-regia digestion was lower than the result of the sodium peroxide fusion dissolution 
and the concentration measured by both dissolution techniques was lower than expected based on a previous analysis of Tank $11 \mathrm{H}$ sludge. ${ }^{9}$ With the thought that the high aluminum concentration in the sample might be suppressing the signal, the samples (both aqua-regia and sodium peroxide fusion) were re-submitted for ICP-ES analysis with higher dilution of the sample by the ADS technician. Tables 3-7 and 3-8 show the results of the re-analysis of the initial set of dissolutions. The aluminum concentration measured in both dissolution methods increased with the higher dilution of the sample, however, the aluminum in the aqua-regia digestions was still lower than the concentration measured in the sodium peroxide fusion dissolution. Examination of the bottles containing these dissolutions in the Shielded Cells showed the presence of undissolved solids in both the aqua-regia and the sodium peroxide fusion dissolutions. The aqua-regia digestions appeared to have more undissolved solids than the sodium peroxide fusion dissolutions. Both dissolution methods were repeated with higher dilution of the sample in the Shielded Cells. Tables 3-9 and 3-10 show the results of the analysis of these samples. Examination of the bottles containing these dissolutions in the Shielded Cells again showed the presence of undissolved solids in the aqua-regia digestions. The bottles containing the repeated sodium peroxide fusion dissolutions appeared clear.

The undissolved solids in the two sets of aqua-regia digestions were filtered from the bottles and submitted to ADS for X-ray diffraction (XRD). The X-ray diffraction spectrum, shown in Figure 3-1, found the sample contained aluminum in the form of Boehmite $(\mathrm{AlO}(\mathrm{OH}))$ and a trace amount of an alumino-silicate phase called Muscovite.

Table 3-4 shows a comparison of the average values from all six dissolution data sets. The data has been colored coded to indicate the data quality flags associated with each set of dissolution data. A comparison of the measured aluminum values from the ICP-ES indicates a dependency on sample concentration in both the aqua-regia and sodium peroxide fusion data sets. Other elements present at high concentrations, such as iron, manganese, and magnesium do not show this dependency. Many of the elements present at lower concentrations show flags indicating concentrations near the detection limit of the method. These flagged values would be expected to have higher uncertainty and usually exhibit higher \%RSD for the replicate analyses.

Table 3-4 also contains a column of recommended values. The recommended values were reported previously. ${ }^{9}$ The recommended values were derived from examination of all the data and data flags associated with each value. The final column in the table provides the number of values used to calculate the recommended value. The recommended values were calculated by averaging the individual replicate values for the dissolution data sets used. For aluminum, only the results of the $3^{\text {rd }}$ sodium peroxide fusion dissolution analysis were used (three values) since this was the only dissolution sample in which the solution appeared clear indicating all of the solids had dissolved. For other major elements in the sample such as, iron, manganese, magnesium, and nickel, all eighteen replicates were used. For the elements calcium, sodium, and zirconium, only the aqua-regia data sets can be used since the sodium peroxide fusion reagents contain these elements. For silicon, only the sodium peroxide fusion data sets can be used since aqua-regia does not dissolve silicon containing compounds well. For the Tank $51 \mathrm{H}$ composite sample only the $3^{\text {rd }}$ sodium peroxide fusion data sets found 
WSRC-TR-2005-00525, REV. 0

values for silicon above the detection limits of the analytical method and therefore, only three values were used to calculate the recommended value. The recommended sulfur value results from the average of $1^{\text {st }}$ and $3^{\text {rd }}$ aqua-regia and sodium peroxide fusion data sets. The $2^{\text {nd }}$ aqua-regia and sodium peroxide fusion data sets were not used to calculate a sulfur value because these sets did not use the low sulfur ICP-ES method that removes known interferences. Many of the elements present in low concentrations in the sample were near the detection limits of the ICP-ES method and more scatter in the data was observed. Additionally, for many of the lower concentration elements in the sample, analysis of the sodium peroxide fusion dissolutions showed high background concentrations in the blank sample.

An Inductively Coupled Plasma-Mass Spectrometry (ICP-MS) analysis was conducted on three sets of dissolutions; the $1^{\text {st }}$ aqua-regia, the $3^{\text {rd }}$ aqua-regia, and the $3^{\text {rd }}$ sodium peroxide fusion data sets. In general the agreement between the three data sets was quite good and all values above the detection limits were used in calculating the recommended values. However, the recommended value for silver did not use the data from the $3^{\text {rd }}$ sodium peroxide fusion due to high background concentrations found in the blank sample. For Cm244 , only one of the nine replicates was above the detection limits of the method, so that single replicate was used as the recommended value. Section 5.5 provides details on the mass values used to calculate the various elements reported.

Radiochemical analysis was only completed on the $1^{\text {st }}$ aqua-regia dissolution. For the gamma emitters, only Cs-137, Eu-154, and Am-241 were above detection limits. The plutonium isotopes from Thenoyltrifluoroacetone Separation/Alpha Counting (TTA) showed high background concentrations in the blank sample for $\mathrm{Pu}-238$ and $\mathrm{Pu}-239 / 240$. However, a comparison of the $\mathrm{Pu}-239 / 240$ value to the sum of the $\mathrm{Pu}-239$ and $\mathrm{Pu}-240$ values from the ICP-MS converted to the same units shows good agreement with difference of less than $1 \%$. Radiochemical analysis was not repeated on the other dissolutions because of the good agreement with ICP-MS results.

In summary, higher uncertainty should be given to the recommended aluminum value, since difficulty was encountered with complete dissolution of this element. Higher uncertainty should be applied to those elements such as sodium, calcium, and zirconium in which only the aqua-regia data sets could be used since the possibility exists that the undissolved solids observed in the aqua-regia digestions could contain low levels of these elements.

Additionally, elements present at low concentrations in the sample, approaching the detection limits of the method, should also be given higher uncertainty. 
WSRC-TR-2005-00525, REV. 0

Table 3-2. Weight Percent Solids, Density, and pH Results from Tank 51 Sample HTF-076-81

\begin{tabular}{|l|c|c|}
\hline Analyte & Average & \%RSD \\
\hline Wt. \% Total Solids & 23.8 wt. \% & $0.6 \%$ \\
\hline Wt.\% Dissolved Solids & 18.6 wt. \% & $1.1 \%$ \\
\hline Wt.\% Insoluble Solids* & 6.41 wt. \% & - \\
\hline Wt.\% Soluble Solids* & 17.4 wt. \% & - \\
\hline Supernate Density & $1.15 \mathrm{~g} / \mathrm{mL}$ & $0.6 \%$ \\
\hline Slurry Density & $1.21 \mathrm{~g} / \mathrm{mL}$ & $1.0 \%$ \\
\hline Supernate pH** & 12.9 & - \\
\hline Slurry pH** & 13.0 & - \\
\hline
\end{tabular}

* Calculated values based on three replicates of the $\mathrm{Wt} \%$ Total Solids and $\mathrm{Wt} \%$ Dissolved Solids. ** Only a single measurement of the $\mathrm{pH}$ was made. 
WSRC-TR-2005-00525, REV. 0

Table 3-3. Composition of the Filtered Supernate from Tank 51 Sample HTF-076-81

\begin{tabular}{|c|c|c|}
\hline Analyte & $\begin{array}{c}\text { Average } \\
\text { (M) }\end{array}$ & $\%$ RSD \\
\hline $\mathrm{NO}_{3}^{-}$(IC) & $1.02 \mathrm{E}+00$ & $1.0 \%$ \\
\hline $\mathrm{NO}_{2}^{-}$(IC) & $9.62 \mathrm{E}-01$ & $1.1 \%$ \\
\hline $\mathrm{PO}_{4}^{3-}$ (IC) & $<6 \mathrm{E}-03$ & - \\
\hline $\mathrm{SO}_{4}{ }^{2-}$ (IC) & $8.76 \mathrm{E}-02$ & $1.3 \%$ \\
\hline $\mathrm{C}_{2} \mathrm{O}_{4}{ }^{2-}(\mathrm{IC})$ & $6.02 \mathrm{E}-03$ & $3.7 \%$ \\
\hline $\mathrm{Cl}^{-}(\mathrm{IC})$ & $3.73 \mathrm{E}-03$ & $13 \%$ \\
\hline $\mathrm{F}^{-}$(IC) & $<6 \mathrm{E}-03$ & - \\
\hline $\mathrm{CHO}_{2}^{-}$(IC) & $<1 \mathrm{E}-02$ & - \\
\hline $\mathrm{OH}_{\text {free }}^{-}(\mathrm{T})$ & $2.70 \mathrm{E}-01$ & $1.3 \%$ \\
\hline Total Base (T) & $6.91 \mathrm{E}-01$ & $5.0 \%$ \\
\hline $\mathrm{CO}_{3}^{2-}(\mathrm{T})$ & $1.94 \mathrm{E}-01$ & $1.7 \%$ \\
\hline $\mathrm{Na}$ (IE) & $2.94 \mathrm{E}+00$ & $0.5 \%$ \\
\hline $\mathrm{Ca}$ (IE) & $1.14 \mathrm{E}-04$ & $1.8 \%$ \\
\hline $\mathrm{Al}$ (IE) & $7.05 \mathrm{E}-02$ & $0.5 \%$ \\
\hline $\mathrm{Cr}$ (IE) & $2.03 \mathrm{E}-03$ & $1.0 \%$ \\
\hline $\mathrm{S}(\mathrm{IE})$ & $8.80 \mathrm{E}-02$ & $2.7 \%$ \\
\hline $\mathrm{Cs}^{137} \mathrm{~d} / \mathrm{m} / \mathrm{mL}$ (GS) & $1.83 \mathrm{E}+08$ & $3.9 \%$ \\
\hline Density $_{\text {supernate }} \mathrm{g} / \mathrm{mL}$ & 1.15 & $0.6 \%$ \\
\hline Wt\% Dissolved Solids & $18.6 \%$ & $1.1 \%$ \\
\hline pH (single measurement) & 12.9 & - \\
\hline
\end{tabular}

Results are the average of three replicates unless noted in the table. 
Table 3-4. Comparison of All Dissolution Data for the Total Dried Solids from Tank 51 Sample HTF-076-81

\begin{tabular}{|c|c|c|c|c|c|c|c|c|c|}
\hline Analyte (Method) & $\begin{array}{c}\text { 1st AQ } \\
\text { Average } \\
(\mathrm{mg} / \mathrm{Kg})\end{array}$ & $\begin{array}{l}\text { 2nd AQ } \\
\text { Average } \\
(\mathrm{mg} / \mathrm{Kg})\end{array}$ & $\begin{array}{c}\text { 3rd AQ } \\
\text { Average } \\
(\mathrm{mg} / \mathrm{Kg}) \\
\end{array}$ & $\begin{array}{c}\text { 1st PF } \\
\text { Average } \\
\text { (mg/Kg) }\end{array}$ & $\begin{array}{c}\text { 2nd PF } \\
\text { Average } \\
(\mathrm{mg} / \mathrm{Kg}) \\
\end{array}$ & $\begin{array}{c}\text { 3rd PF } \\
\text { Average } \\
(\mathrm{mg} / \mathrm{Kg})\end{array}$ & $\begin{array}{c}\text { Recommended } \\
\text { Value to Use } \\
(\mathrm{mg} / \mathrm{Kg})^{\mathrm{a}}\end{array}$ & $\%$ RSD & $\begin{array}{c}\text { No. of } \\
\text { Values Used }\end{array}$ \\
\hline Ag (IE) & $<4.5 \mathrm{E}+01$ & $<4.5 \mathrm{E}+02$ & $<6.9 \mathrm{E}+01$ & $2.81 \mathrm{E}+02$ & $1.34 \mathrm{E}+03 * *$ & $6.33 \mathrm{E}+02 *$ & Use MS data & - & - \\
\hline Al (IE) & $4.95 \mathrm{E}+04$ & $5.09 \mathrm{E}+04$ & $5.99 \mathrm{E}+04$ & $6.84 \mathrm{E}+04$ & $7.14 \mathrm{E}+04$ & $7.49 \mathrm{E}+04$ & $7.49 \mathrm{E}+04$ & $3.5 \%$ & 3 \\
\hline B (IE) & $<2.2 \mathrm{E}+02$ & $<2.2 \mathrm{E}+03$ & $<5.3 \mathrm{E}+01$ & $<2.1 \mathrm{E}+02$ & $<2.1 \mathrm{E}+03$ & $<4.8 \mathrm{E}+02$ & $<4.8 \mathrm{E}+02$ & - & - \\
\hline $\mathrm{Ba}$ (IE) & $2.05 \mathrm{E}+02$ & $<9.5 \mathrm{E}+02$ & $2.27 \mathrm{E}+02$ & $2.60 \mathrm{E}+02$ & $1.04 \mathrm{E}+03 *$ & $3.45 \mathrm{E}+02$ & $2.59 \mathrm{E}+02$ & $22 \%$ & 12 \\
\hline $\mathrm{Ca}$ (IE) & $3.88 \mathrm{E}+03$ & $3.77 \mathrm{E}+03$ & $4.19 \mathrm{E}+03$ & - & - & - & $3.94 \mathrm{E}+03$ & $6.1 \%$ & 3 \\
\hline $\mathrm{Cd}$ (IE) & $7.07 \mathrm{E}+01$ & $2.33 \mathrm{E}+02 *$ & $1.27 \mathrm{E}+02$ & $8.95 \mathrm{E}+01$ & $<1.7 \mathrm{E}+02$ & $<1.4 \mathrm{E}+02$ & $<1.4 \mathrm{E}+02$ & - & - \\
\hline $\mathrm{Ce}$ (IE) & $<5.6 \mathrm{E}+02$ & $<5.6 \mathrm{E}+03$ & $5.98 \mathrm{E}+02$ & $1.73 \mathrm{E}+03$ & $2.29 \mathrm{E}+04$ & $8.61 \mathrm{E}+03$ & $<5.6 \mathrm{E}+02$ & - & - \\
\hline $\mathrm{Cr}$ (IE) & $3.87 \mathrm{E}+02$ & $<1.1 \mathrm{E}+03$ & $4.21 \mathrm{E}+02$ & $1.94 \mathrm{E}+03$ & $2.63 \mathrm{E}+03 * *$ & $3.58 \mathrm{E}+02 *$ & $4.21 \mathrm{E}+02$ & $9.0 \%$ & 3 \\
\hline $\mathrm{Cu}$ (IE) & $<4.1 \mathrm{E}+01$ & $<4.1 \mathrm{E}+02$ & $1.39 \mathrm{E}+02$ & $9.68 \mathrm{E}+01$ & $<4.0 \mathrm{E}+02$ & $1.85 \mathrm{E}+02$ & $<4.1 \mathrm{E}+01$ & - & - \\
\hline $\mathrm{Fe}$ (IE) & $3.55 \mathrm{E}+04$ & $3.67 \mathrm{E}+04$ & $3.68 \mathrm{E}+04$ & $3.74 \mathrm{E}+04$ & $3.91 \mathrm{E}+04$ & $3.74 \mathrm{E}+04$ & $3.72 \mathrm{E}+04$ & $7.1 \%$ & 18 \\
\hline K (IE) & $4.25 \mathrm{E}+03 *$ & $<3.2 \mathrm{E}+04$ & $1.53 \mathrm{E}+03$ & $7.89 \mathrm{E}+03$ & $7.04 \mathrm{E}+04 * *$ & $2.26 \mathrm{E}+04$ & $1.53 \mathrm{E}+03$ & $1.0 \%$ & 3 \\
\hline La (IE) & $<2.1 \mathrm{E}+02$ & $<2.1 \mathrm{E}+03$ & $2.14 \mathrm{E}+02$ & $3.33 \mathrm{E}+02$ & $4.42 \mathrm{E}+03 * *$ & $1.27 \mathrm{E}+03$ & $2.74 \mathrm{E}+02$ & $1.0 \%$ & 6 \\
\hline $\mathrm{Li}$ (IE) & $<2.0 \mathrm{E}+02$ & $<2.0 \mathrm{E}+03$ & $6.87 \mathrm{E}+01 *$ & $<1.9 \mathrm{E}+02$ & $4.29 \mathrm{E}+03 * *$ & $1.19 \mathrm{E}+03$ & $<2.0 \mathrm{E}+02$ & - & - \\
\hline $\mathrm{Mg}$ (IE) & $1.48 \mathrm{E}+03$ & $1.39 \mathrm{E}+03$ & $1.55 \mathrm{E}+03$ & $1.38 \mathrm{E}+03$ & $1.37 \mathrm{E}+03$ & $1.36 \mathrm{E}+03$ & $1.42 \mathrm{E}+03$ & $7.5 \%$ & 18 \\
\hline Mn (IE) & $8.94 \mathrm{E}+03$ & $9.31 \mathrm{E}+03$ & $9.12 \mathrm{E}+03$ & $8.22 \mathrm{E}+03$ & $8.59 \mathrm{E}+03$ & $9.01 \mathrm{E}+03$ & $8.86 \mathrm{E}+03$ & $5.4 \%$ & 18 \\
\hline Mo (IE) & $<5.5 \mathrm{E}+02$ & $<5.5 \mathrm{E}+03$ & $1.62 \mathrm{E}+02 * *$ & $<5.5 \mathrm{E}+02$ & $<5.4 \mathrm{E}+03$ & $1.36 \mathrm{E}+03 *$ & $<5.5 \mathrm{E}+02$ & - & - \\
\hline $\mathrm{Na}$ (IE) & $2.33 \mathrm{E}+05$ & $2.42 \mathrm{E}+05$ & $2.23 \mathrm{E}+05$ & - & - & - & $2.33 \mathrm{E}+05$ & $4.5 \%$ & 3 \\
\hline
\end{tabular}

${ }^{a}$ These values were published in reference 6 .

* Single Value $\quad * *$ Average of two replicates

Data Quality Flags: $\quad \mathrm{U}_{\mathrm{E}}-$ result is less than $10 \mathrm{X}$ the detection limit

$\mathrm{U}_{\mathrm{B}}$ - blank result greater than $5 \%$ of sample result

Divide $\mathrm{mg} / \mathrm{Kg}$ values by $1 \mathrm{E}+04$ to convert to $\mathrm{wt} \%$ basis

$\mathrm{U}_{\mathrm{R}}-\% \mathrm{RSD}$ greater than $15 \%$

$\mathrm{U}_{\mathrm{G}}-$ glass std result greater than $15 \%$ from expected result 
WSRC-TR-2005-00525, REV. 0

Table 3-4. Comparison of All Dissolution Data for Total Dried Solids from Tank 51 Sample HTF-076-81 (continued)

\begin{tabular}{|c|c|c|c|c|c|c|c|c|c|}
\hline Analyte (Method) & $\begin{array}{c}\text { 1st AQ } \\
\text { Average } \\
(\mathrm{mg} / \mathrm{Kg}) \\
\end{array}$ & $\begin{array}{l}\text { 2nd AQ } \\
\text { Average } \\
\text { (mg/Kg) }\end{array}$ & $\begin{array}{c}\text { 3rd AQ } \\
\text { Average } \\
\text { (mg/Kg) }\end{array}$ & $\begin{array}{c}\text { 1st PF } \\
\text { Average } \\
(\mathrm{mg} / \mathrm{Kg}) \\
\end{array}$ & $\begin{array}{l}\text { 2nd PF } \\
\text { Average } \\
(\mathrm{mg} / \mathrm{Kg})\end{array}$ & $\begin{array}{c}\text { 3rd PF } \\
\text { Average } \\
(\mathrm{mg} / \mathrm{Kg})\end{array}$ & \begin{tabular}{|c|} 
Recommended \\
Value to Use \\
$(\mathrm{mg} / \mathrm{Kg})^{\mathrm{a}}$
\end{tabular} & $\%$ RSD & $\begin{array}{c}\text { No. of } \\
\text { Values Used }\end{array}$ \\
\hline $\mathrm{Ni}$ (IE) & $3.20 \mathrm{E}+03$ & $2.96 \mathrm{E}+03$ & $3.26 \mathrm{E}+03$ & $3.29 \mathrm{E}+03$ & $3.69 \mathrm{E}+03$ & $3.11 \mathrm{E}+03$ & $3.25 \mathrm{E}+03$ & $9.6 \%$ & 18 \\
\hline$P(\mathrm{IE})$ & $1.15 \mathrm{E}+03^{*}$ & $<1.1 \mathrm{E}+04$ & $6.99 \mathrm{E}+02$ & $<1.1 \mathrm{E}+03$ & $<1.1 \mathrm{E}+04$ & $<2.4 \mathrm{E}+03$ & $<2.4 \mathrm{E}+03$ & - & - \\
\hline $\mathrm{Pb}$ (IE) & $<3.1 \mathrm{E}+03$ & $<3.1 \mathrm{E}+04$ & $<2.6 \mathrm{E}+02$ & $<3.1 \mathrm{E}+03$ & $<3.1 \mathrm{E}+04$ & $<2.4 \mathrm{E}+03$ & $<3.1 \mathrm{E}+03$ & - & - \\
\hline S (IE) & $9.22 \mathrm{E}+03$ & $8.45 \mathrm{E}+03$ & $8.61 \mathrm{E}+03$ & $1.12 \mathrm{E}+04$ & $1.38 \mathrm{E}+04$ & $8.82 \mathrm{E}+03$ & $9.46 \mathrm{E}+03$ & $12 \%$ & 12 \\
\hline $\mathrm{Sb}$ (IE) & $<4.8 \mathrm{E}+02$ & $<4.8 \mathrm{E}+03$ & $2.90 \mathrm{E}+02$ & $4.95 \mathrm{E}+02$ & $<4.7 \mathrm{E}+03$ & $1.63 \mathrm{E}+03$ & $<4.7 \mathrm{E}+03$ & - & - \\
\hline Si (IE) & - & - & - & $<2.0 \mathrm{E}+03$ & $<2.0 \mathrm{E}+04$ & $8.81 \mathrm{E}+03$ & $8.81 \mathrm{E}+03$ & $4.7 \%$ & 3 \\
\hline $\mathrm{Sn}$ (IE) & $<1.2 \mathrm{E}+03$ & $<1.2 \mathrm{E}+04$ & $<3.0 \mathrm{E}+02$ & $1.25 \mathrm{E}+03 * *$ & $1.53 \mathrm{E}+04 * *$ & $3.31 \mathrm{E}+03 *$ & $<1.2 \mathrm{E}+04$ & - & - \\
\hline $\mathrm{Sr}$ (IE) & $8.13 \mathrm{E}+02$ & $1.11 \mathrm{E}+03$ & $9.09 \mathrm{E}+02$ & $1.22 \mathrm{E}+03$ & $3.61 \mathrm{E}+03$ & $2.00 \mathrm{E}+03$ & $9.44 \mathrm{E}+02$ & $1.0 \%$ & 9 \\
\hline Ti (IE) & $4.57 \mathrm{E}+01$ & $<1.2 \mathrm{E}+02$ & $4.78 \mathrm{E}+01$ & $5.60 \mathrm{E}+01$ & $<1.2 \mathrm{E}+02$ & $2.84 \mathrm{E}+01 *$ & $4.98 \mathrm{E}+01$ & $13 \%$ & 9 \\
\hline U (IE) & $8.65 \mathrm{E}+03$ & $<8.1 \mathrm{E}+03$ & $9.06 \mathrm{E}+03$ & $9.54 \mathrm{E}+03$ & $1.80 \mathrm{E}+04$ & $1.40 \mathrm{E}+04$ & $9.08 \mathrm{E}+03$ & $5.3 \%$ & 9 \\
\hline $\mathrm{Zn}$ (IE) & $3.91 \mathrm{E}+01$ & $<2.9 \mathrm{E}+02$ & $1.46 \mathrm{E}+02$ & $1.75 \mathrm{E}+02$ & $8.50 \mathrm{E}+02$ & $3.39 \mathrm{E}+02$ & $<2.9 \mathrm{E}+02$ & - & - \\
\hline $\mathrm{Zr}$ (IE) & $4.66 \mathrm{E}+02$ & $4.03 \mathrm{E}+02$ & $4.19 \mathrm{E}+02$ & - & - & - & $4.29 \mathrm{E}+02$ & $18 \%$ & 9 \\
\hline $\mathrm{Hg}(\mathrm{CV})$ & $1.34 \mathrm{E}+04$ & - & - & - & - & - & $1.34 \mathrm{E}+04$ & $1.9 \%$ & 3 \\
\hline
\end{tabular}

${ }^{a}$ These values were published in reference 6 .

* Single Value ** Average of two replicates

Data Quality Flags: $\quad \mathrm{U}_{\mathrm{E}}$ - result is less than $10 \mathrm{X}$ the detection limit

$\mathrm{U}_{\mathrm{B}}$ - blank result greater than $5 \%$ of sample result

Divide $\mathrm{mg} / \mathrm{Kg}$ values by $1 \mathrm{E}+04$ to convert to $\mathrm{wt} \%$ basis

$\mathrm{U}_{\mathrm{R}}-\% \mathrm{RSD}$ greater than $15 \%$

$\mathrm{U}_{\mathrm{G}}$ - glass std result greater than $15 \%$ from expected result 
WSRC-TR-2005-00525, REV. 0

Table 3-4. Comparison of All Dissolution Data for Total Dried Solids from Tank 51 Sample HTF-076-81 (continued)

\begin{tabular}{|c|c|c|c|c|c|c|c|c|c|}
\hline Analyte (Method) & $\begin{array}{c}\text { 1st AQ } \\
\text { Average } \\
(\mathrm{mg} / \mathrm{Kg}) \\
\end{array}$ & $\begin{array}{l}\text { 2nd AQ } \\
\text { Average } \\
\text { (mg/Kg) }\end{array}$ & $\begin{array}{l}\text { 3rd AQ } \\
\text { Average } \\
(\mathrm{mg} / \mathrm{Kg}) \\
\end{array}$ & $\begin{array}{c}\text { 1st PF } \\
\text { Average } \\
(\mathrm{mg} / \mathrm{Kg}) \\
\end{array}$ & $\begin{array}{c}\text { 2nd PF } \\
\text { Average } \\
(\mathrm{mg} / \mathrm{Kg}) \\
\end{array}$ & $\begin{array}{c}\text { 3rd PF } \\
\text { Average } \\
(\mathrm{mg} / \mathrm{Kg}) \\
\end{array}$ & $\begin{array}{c}\text { Recommended } \\
\text { Value to Use } \\
(\mathrm{mg} / \mathrm{Kg})^{\mathrm{a}}\end{array}$ & $\%$ RSD & $\begin{array}{c}\text { No. of } \\
\text { Values Used }\end{array}$ \\
\hline Th (IM) & $3.28 \mathrm{E}+02$ & - & $3.24 \mathrm{E}+02$ & - & - & $3.39 \mathrm{E}+02$ & $3.30 \mathrm{E}+02$ & $2.6 \%$ & 9 \\
\hline $\mathrm{Ru}$ (IM) & $1.99 \mathrm{E}+02$ & - & $<9.5 \mathrm{E}+01$ & - & - & $4.21 \mathrm{E}+02$ & $3.10 \mathrm{E}+02$ & $39 \%$ & 6 \\
\hline Ag (IM) & $1.33 \mathrm{E}+01$ & - & $<4.2 \mathrm{E}+01$ & - & - & $3.55 \mathrm{E}+01$ & $1.36 \mathrm{E}+01$ & $1.2 \%$ & 3 \\
\hline $\mathrm{Rh}$ (IM) & $4.40 \mathrm{E}+01$ & - & $4.59 \mathrm{E}+01$ & - & - & $4.79 \mathrm{E}+01$ & $4.59 \mathrm{E}+01$ & $5.9 \%$ & 9 \\
\hline $\mathrm{Pd}$ (IM) & $7.46 \mathrm{E}+00$ & - & $7.41 \mathrm{E}+00 *$ & - & - & $7.39 \mathrm{E}+00$ & $7.67 \mathrm{E}+00$ & $5.4 \%$ & 6 \\
\hline $\mathrm{Tc}^{99}$ (IM) & $9.12 \mathrm{E}+00$ & - & $<1.9 \mathrm{E}+01$ & - & - & $1.12 \mathrm{E}+01$ & $1.01 \mathrm{E}+01$ & $11 \%$ & 6 \\
\hline $\mathrm{Np}^{237}$ (IM) & $5.74 \mathrm{E}+00$ & - & $6.86 \mathrm{E}+00$ & - & - & $5.79 \mathrm{E}+00$ & $6.13 \mathrm{E}+00$ & $11 \%$ & 9 \\
\hline $\mathrm{Pu}^{239}(\mathrm{IM})$ & $4.80 \mathrm{E}+01$ & - & $4.29 \mathrm{E}+01$ & - & - & $4.86 \mathrm{E}+01$ & $4.65 \mathrm{E}+01$ & $7.0 \%$ & 9 \\
\hline $\mathrm{Pu}^{240}$ (IM) & $6.22 \mathrm{E}+00$ & - & $6.67 \mathrm{E}+00$ & - & - & $6.68 \mathrm{E}+00$ & $6.52 \mathrm{E}+00$ & $7.5 \%$ & 9 \\
\hline $\mathrm{Am}^{241} / \mathrm{Pu}^{241}$ (IM) & $3.04 \mathrm{E}+00$ & - & $<9.1 \mathrm{E}-01$ & - & - & $2.87 \mathrm{E}+00$ & $2.95 \mathrm{E}+00$ & $4.4 \%$ & 9 \\
\hline $\mathrm{Am}^{243}$ (IM) & $8.39 \mathrm{E}-01$ & - & $<9.1 \mathrm{E}-01$ & - & - & $7.99 \mathrm{E}-01$ & $8.19 \mathrm{E}-01$ & $8.9 \%$ & 6 \\
\hline $\mathrm{Cm}^{244}$ (IM) & $2.60 \mathrm{E}-01 *$ & - & $<9.1 \mathrm{E}-01$ & - & - & $<4.6 \mathrm{E}-01$ & $2.60 \mathrm{E}-01 *$ & - & 1 \\
\hline $\mathrm{U}^{233}$ (IM) & $8.92 \mathrm{E}-01$ & - & $<9.1 \mathrm{E}-01$ & - & - & $8.28 \mathrm{E}-01$ & $8.60 \mathrm{E}-01$ & $8.0 \%$ & 6 \\
\hline $\mathrm{U}^{234}$ (IM) & $3.74 \mathrm{E}+00$ & - & $4.56 \mathrm{E}+00 *$ & - & - & $3.94 \mathrm{E}+00$ & $3.84 \mathrm{E}+00$ & $4.0 \%$ & 6 \\
\hline $\mathrm{U}^{235}$ (IM) & $6.81 \mathrm{E}+01$ & - & $6.55 \mathrm{E}+01$ & - & - & $6.78 \mathrm{E}+01$ & $6.72 \mathrm{E}+01$ & $3.3 \%$ & 9 \\
\hline $\mathrm{U}^{236}$ (IM) & $5.43 \mathrm{E}+00$ & - & $6.08 \mathrm{E}+00$ & - & - & $5.65 \mathrm{E}+00$ & $5.72 \mathrm{E}+00$ & $7.0 \%$ & 9 \\
\hline $\mathrm{U}^{238}$ (IM) & $8.06 \mathrm{E}+03$ & - & $8.06 \mathrm{E}+03$ & - & - & $8.44 \mathrm{E}+03$ & $8.18 \mathrm{E}+03$ & $2.9 \%$ & 9 \\
\hline U-total (sum) & $8.13 \mathrm{E}+03$ & - & $8.13 \mathrm{E}+03$ & - & - & $8.51 \mathrm{E}+03$ & $8.32 \mathrm{E}+03$ & $3.2 \%$ & 6 \\
\hline
\end{tabular}

a These values were published in reference 6 .

* Single Value Divide $\mathrm{mg} / \mathrm{Kg}$ values by $1 \mathrm{E}+04$ to convert to $\mathrm{wt} \%$ basis

Data Quality Flags: $\quad \mathrm{U}_{\mathrm{E}}$ - result is less than $10 \mathrm{X}$ the detection limit

$\mathrm{U}_{\mathrm{B}}$ - blank result greater than $5 \%$ of sample result $\quad \mathrm{U}_{\mathrm{G}}-$ glass std result greater than $15 \%$ from expected result 
WSRC-TR-2005-00525, REV. 0

Table 3-4. Comparison of All Dissolution Data for Total Dried Solids from Tank 51 Sample HTF-076-81 (continued)

\begin{tabular}{|c|c|c|c|c|c|c|c|c|c|}
\hline Analyte (Method) & $\begin{array}{c}\text { 1st AQ } \\
\text { Average } \\
(\mathbf{m C i} / \mathbf{K g})\end{array}$ & $\begin{array}{c}\text { 2nd AQ } \\
\text { Average } \\
(\mathbf{m C i} / \mathbf{K g})\end{array}$ & $\begin{array}{c}\text { 3rd AQ } \\
\text { Average } \\
(\mathrm{mCi} / \mathrm{Kg}) \\
\end{array}$ & $\begin{array}{c}\text { 1st PF } \\
\text { Average } \\
(\mathbf{m C i} / \mathbf{K g})\end{array}$ & $\begin{array}{c}\text { 2nd PF } \\
\text { Average } \\
(\mathrm{mCi} / \mathrm{Kg})\end{array}$ & $\begin{array}{c}\text { 3rd PF } \\
\text { Average } \\
(\mathbf{m C i} / \mathbf{K g})\end{array}$ & $\begin{array}{c}\text { Recommended } \\
\text { Value to Use } \\
(\mathrm{mCi} / \mathrm{Kg})^{a}\end{array}$ & $\%$ RSD & $\begin{array}{c}\text { No. of } \\
\text { Values Used } \\
\end{array}$ \\
\hline $\mathrm{Cs}^{137}$ (GS) & $3.47 \mathrm{E}+02$ & - & - & - & - & - & $3.47 \mathrm{E}+02$ & $3.2 \%$ & 3 \\
\hline $\mathrm{Cs}^{134}(\mathrm{GS})$ & $<1.5 \mathrm{E}+00$ & - & - & - & - & - & $<1.5 \mathrm{E}+00$ & - & - \\
\hline $\mathrm{Eu}^{154}(\mathrm{GS})$ & $1.46 \mathrm{E}+01$ & - & - & - & - & - & $1.46 \mathrm{E}+01$ & $11 \%$ & 3 \\
\hline $\mathrm{Eu}^{155}(\mathrm{GS})$ & $<5.9 \mathrm{E}+00$ & - & - & - & - & - & $<5.9 \mathrm{E}+00$ & - & - \\
\hline $\mathrm{Co}^{60}(\mathrm{GS})$ & $<1.3 \mathrm{E}+00$ & - & - & - & - & - & $<1.3 \mathrm{E}+00$ & - & - \\
\hline $\mathrm{Ru}^{106}(\mathrm{GS})$ & $<1.1 \mathrm{E}+01$ & - & - & - & - & - & $<1.1 \mathrm{E}+01$ & - & - \\
\hline $\mathrm{Ce}^{144}(\mathrm{GS})$ & $<1.3 \mathrm{E}+01$ & - & - & - & - & - & $<1.3 \mathrm{E}+01$ & - & - \\
\hline $\mathrm{Sb}^{125}(\mathrm{GS})$ & $<7.8 \mathrm{E}+00$ & - & - & - & - & - & $<7.8 \mathrm{E}+00$ & - & - \\
\hline $\mathrm{Am}^{241}(\mathrm{GS})$ & $7.89 \mathrm{E}+00$ & - & - & - & - & - & $7.89 \mathrm{E}+00$ & $3.9 \%$ & 3 \\
\hline $\mathrm{Sr}^{90} \quad(\mathrm{BS})$ & $4.82 \mathrm{E}+03$ & - & - & - & - & - & $4.82 \mathrm{E}+03$ & $12 \%$ & 3 \\
\hline $\mathrm{Pu}^{239 / 240}$ (TTA) & $4.34 \mathrm{E}+00$ & - & - & - & - & - & $4.34 \mathrm{E}+00$ & $10 \%$ & 3 \\
\hline $\mathrm{Pu}^{241}$ (TTA) & $6.12 \mathrm{E}+01$ & - & - & - & - & - & $6.12 \mathrm{E}+01$ & $6.7 \%$ & 3 \\
\hline
\end{tabular}

${ }^{a}$ These values were published in reference 6 .

Data Quality Flags: $\quad \mathrm{U}_{\mathrm{E}}$ - result is less than $10 \mathrm{X}$ the detection limit

$\mathrm{U}_{\mathrm{B}}$ - blank result greater than $5 \%$ of sample result

$\mathrm{U}_{\mathrm{R}}$ - \%RSD greater than $15 \%$

$\mathrm{U}_{\mathrm{G}}-$ glass std result greater than $15 \%$ from expected result 
WSRC-TR-2005-00525, REV. 0

Table 3-5. Composition of the $1^{\text {st }}$ Aqua Regia Dissolution of Solids from Tank 51 Sample HTF-076-81

\begin{tabular}{|c|c|c|c|c|c|c|c|c|c|}
\hline Analyte (Method) & $\begin{array}{c}\text { 1st } \\
\text { Replicate } \\
(\mathrm{mg} / \mathrm{Kg})\end{array}$ & $\begin{array}{c}\text { 2nd } \\
\text { Replicate } \\
(\mathrm{mg} / \mathrm{Kg})\end{array}$ & $\begin{array}{c}\text { 3rd } \\
\text { Replicate } \\
(\mathrm{mg} / \mathrm{Kg})\end{array}$ & $\begin{array}{l}\text { Average } \\
\text { (mg/Kg) }\end{array}$ & \%RSD & $\begin{array}{c}\text { Blank } \\
(\mathbf{m g} / \mathbf{K g})\end{array}$ & $\begin{array}{l}\text { Analyzed } \\
\text { Glass Std } \\
(\mathrm{mg} / \mathrm{Kg}) \\
\end{array}$ & \begin{tabular}{|c|}
$\begin{array}{c}\text { Glass Std } \\
\text { Composition } \\
(\mathrm{mg} / \mathrm{Kg})\end{array}$ \\
\end{tabular} & $\begin{array}{c}\text { Data } \\
\text { Quality } \\
\text { Flags } \\
\end{array}$ \\
\hline Ag (IE) & $<4.6 \mathrm{E}+01$ & $<4.4 \mathrm{E}+01$ & $<4.6 \mathrm{E}+01$ & $<4.5 \mathrm{E}+01$ & - & $<4.4 \mathrm{E}+01$ & $<4.1 \mathrm{E}+01$ & - & - \\
\hline $\mathrm{Al}$ (IE) & $5.54 \mathrm{E}+04$ & $4.48 \mathrm{E}+04$ & $4.83 \mathrm{E}+04$ & $4.95 \mathrm{E}+04$ & $11 \%$ & $<1.7 \mathrm{E}+02$ & $2.11 \mathrm{E}+04$ & $2.50 \mathrm{E}+04$ & $\mathrm{U}_{\mathrm{G}}$ \\
\hline B (IE) & $<2.2 \mathrm{E}+02$ & $<2.1 \mathrm{E}+02$ & $<2.2 \mathrm{E}+02$ & $<2.2 \mathrm{E}+02$ & - & $<2.1 \mathrm{E}+02$ & $2.47 \mathrm{E}+04$ & $2.69 \mathrm{E}+04$ & - \\
\hline $\mathrm{Ba}$ (IE) & $2.17 \mathrm{E}+02$ & $1.97 \mathrm{E}+02$ & $2.00 \mathrm{E}+02$ & $2.05 \mathrm{E}+02$ & $5.3 \%$ & $<9.2 \mathrm{E}+01$ & $7.59 \mathrm{E}+02$ & $7.90 \mathrm{E}+02$ & $\mathrm{U}_{\mathrm{E}}$ \\
\hline $\mathrm{Ca}$ (IE) & $4.10 \mathrm{E}+03$ & $3.65 \mathrm{E}+03$ & $3.88 \mathrm{E}+03$ & $3.88 \mathrm{E}+03$ & $5.8 \%$ & $<3.6 \mathrm{E}+01$ & $9.78 \mathrm{E}+03$ & $1.02 \mathrm{E}+04$ & $\mathrm{U}_{\mathrm{R}}$ \\
\hline $\mathrm{Cd}$ (IE) & $8.13 \mathrm{E}+01$ & $6.84 \mathrm{E}+01$ & $6.25 \mathrm{E}+01$ & $7.07 \mathrm{E}+01$ & $14 \%$ & $<1.7 \mathrm{E}+01$ & $<1.6 \mathrm{E}+01$ & - & $\mathrm{U}_{\mathrm{E}}$ \\
\hline $\mathrm{Ce}$ (IE) & $<5.6 \mathrm{E}+02$ & $<5.4 \mathrm{E}+02$ & $<5.6 \mathrm{E}+02$ & $<5.6 \mathrm{E}+02$ & - & $<5.4 \mathrm{E}+02$ & $<5.0 \mathrm{E}+02$ & - & - \\
\hline $\mathrm{Cr}$ (IE) & $3.85 \mathrm{E}+02$ & $3.60 \mathrm{E}+02$ & $4.17 \mathrm{E}+02$ & $3.87 \mathrm{E}+02$ & $7.3 \%$ & $<1.0 \mathrm{E}+02$ & $4.41 \mathrm{E}+02$ & $6.40 \mathrm{E}+02$ & $\mathrm{U}_{\mathrm{E}} \mathrm{U}_{\mathrm{G}}$ \\
\hline $\mathrm{Cu}$ (IE) & $<4.2 \mathrm{E}+01$ & $<4.0 \mathrm{E}+01$ & $<4.2 \mathrm{E}+01$ & $<4.1 \mathrm{E}+01$ & - & $<4.0 \mathrm{E}+01$ & $<3.7 \mathrm{E}+01$ & $3.00 \mathrm{E}+01$ & $\mathrm{U}_{\mathrm{G}}$ \\
\hline $\mathrm{Fe}(\mathrm{IE})$ & $3.73 \mathrm{E}+04$ & $3.38 \mathrm{E}+04$ & $3.54 \mathrm{E}+04$ & $3.55 \mathrm{E}+04$ & $4.9 \%$ & $<4.8 \mathrm{E}+01$ & $9.37 \mathrm{E}+04$ & $9.79 \mathrm{E}+04$ & - \\
\hline $\mathrm{K}$ (IE) & $4.25 \mathrm{E}+03$ & $<3.1 \mathrm{E}+03$ & $<3.2 \mathrm{E}+03$ & $4.25 \mathrm{E}+03 *$ & - & $<3.1 \mathrm{E}+03$ & $2.05 \mathrm{E}+04$ & $2.26 \mathrm{E}+04$ & $\mathrm{U}_{\mathrm{E}} \mathrm{U}_{\mathrm{G}}$ \\
\hline La (IE) & $<2.2 \mathrm{E}+02$ & $<2.1 \mathrm{E}+02$ & $<2.2 \mathrm{E}+02$ & $<2.1 \mathrm{E}+02$ & - & $<2.1 \mathrm{E}+02$ & $<1.9 \mathrm{E}+02$ & - & - \\
\hline $\mathrm{Li}$ (IE) & $<2.0 \mathrm{E}+02$ & $<1.9 \mathrm{E}+02$ & $<2.0 \mathrm{E}+02$ & $<2.0 \mathrm{E}+02$ & - & $<1.9 \mathrm{E}+02$ & $1.40 \mathrm{E}+04$ & $1.49 \mathrm{E}+04$ & - \\
\hline $\mathrm{Mg}$ (IE) & $1.56 \mathrm{E}+03$ & $1.41 \mathrm{E}+03$ & $1.48 \mathrm{E}+03$ & $1.48 \mathrm{E}+03$ & $5.0 \%$ & $<1.6 \mathrm{E}+01$ & $4.89 \mathrm{E}+03$ & $5.20 \mathrm{E}+03$ & - \\
\hline Mn (IE) & $9.38 \mathrm{E}+03$ & $8.52 \mathrm{E}+03$ & $8.92 \mathrm{E}+03$ & $8.94 \mathrm{E}+03$ & $4.8 \%$ & $<8.0 \mathrm{E}+00$ & $1.40 \mathrm{E}+04$ & $1.46 \mathrm{E}+04$ & - \\
\hline Mo (IE) & $<5.5 \mathrm{E}+02$ & $<5.3 \mathrm{E}+02$ & $<5.5 \mathrm{E}+02$ & $<5.5 \mathrm{E}+02$ & - & $<5.3 \mathrm{E}+02$ & $<4.9 \mathrm{E}+02$ & - & - \\
\hline $\mathrm{Na}$ (IE) & $2.43 \mathrm{E}+05$ & $2.23 \mathrm{E}+05$ & $2.32 \mathrm{E}+05$ & $2.33 \mathrm{E}+05$ & $4.3 \%$ & $<1.4 \mathrm{E}+03$ & $7.96 \mathrm{E}+04$ & $8.52 \mathrm{E}+04$ & - \\
\hline
\end{tabular}

* Single Value Divide $\mathrm{mg} / \mathrm{Kg}$ values by $1 \mathrm{E}+04$ to convert to $\mathrm{wt} \%$ basis

Data Quality Flags: $\quad \mathrm{U}_{\mathrm{E}}$ - result is less than 10X the detection limit

$\mathrm{U}_{\mathrm{B}}$ - blank result greater than $5 \%$ of sample result

$\mathrm{U}_{\mathrm{R}}-\%$ RSD greater than $15 \%$

$\mathrm{U}_{\mathrm{G}}-$ glass std result greater than $15 \%$ from expected result 
WSRC-TR-2005-00525, REV. 0

Table 3-5. Composition of the $1^{\text {st }}$ Aqua Regia Dissolution of Solids from Tank 51 Sample HTF-076-81 (continued)

\begin{tabular}{|c|c|c|c|c|c|c|c|c|c|}
\hline Analyte (Method) & $\begin{array}{c}\text { 1st } \\
\text { Replicate } \\
(\mathrm{mg} / \mathrm{Kg})\end{array}$ & $\begin{array}{c}\text { 2nd } \\
\text { Replicate } \\
(\mathrm{mg} / \mathrm{Kg})\end{array}$ & $\begin{array}{c}\text { 3rd } \\
\text { Replicate } \\
(\mathrm{mg} / \mathrm{Kg})\end{array}$ & $\begin{array}{l}\text { Average } \\
\text { (mg/Kg) }\end{array}$ & \%RSD & $\begin{array}{c}\text { Blank } \\
(\mathrm{mg} / \mathrm{Kg})\end{array}$ & $\begin{array}{c}\text { Analyzed } \\
\text { Glass Std } \\
(\mathrm{mg} / \mathrm{Kg})\end{array}$ & $\begin{array}{c}\text { Glass Std } \\
\text { Composition } \\
(\mathrm{mg} / \mathrm{Kg})\end{array}$ & $\begin{array}{c}\text { Data } \\
\text { Quality } \\
\text { Flags } \\
\end{array}$ \\
\hline $\mathrm{Ni}$ (IE) & $3.30 \mathrm{E}+03$ & $3.13 \mathrm{E}+03$ & $3.17 \mathrm{E}+03$ & $3.20 \mathrm{E}+03$ & $2.8 \%$ & $<1.6 \mathrm{E}+02$ & $8.15 \mathrm{E}+03$ & $8.27 \mathrm{E}+03$ & - \\
\hline $\mathrm{P}$ (IE) & $<1.1 \mathrm{E}+03$ & $1.15 \mathrm{E}+03$ & $<1.1 \mathrm{E}+03$ & $1.15 \mathrm{E}+03 *$ & - & $<1.1 \mathrm{E}+03$ & $1.10 \mathrm{E}+03$ & $1.10 \mathrm{E}+03$ & $\mathrm{U}_{\mathrm{E}}$ \\
\hline $\mathrm{Pb}$ (IE) & $<3.2 \mathrm{E}+03$ & $<3.0 \mathrm{E}+03$ & $<3.2 \mathrm{E}+03$ & $<3.1 \mathrm{E}+03$ & - & $<3.0 \mathrm{E}+03$ & $<2.8 \mathrm{E}+03$ & - & - \\
\hline S (IE) & $9.71 \mathrm{E}+03$ & $8.88 \mathrm{E}+03$ & $9.08 \mathrm{E}+03$ & $9.22 \mathrm{E}+03$ & $4.7 \%$ & $5.56 \mathrm{E}+01$ & $3.28 \mathrm{E}+02$ & - & - \\
\hline $\mathrm{Sb}$ (IE) & $<4.8 \mathrm{E}+02$ & $<4.6 \mathrm{E}+02$ & $<4.8 \mathrm{E}+02$ & $<4.8 \mathrm{E}+02$ & - & $<4.6 \mathrm{E}+02$ & $<4.3 \mathrm{E}+02$ & - & - \\
\hline $\mathrm{Si}$ (IE) & $<2.0 \mathrm{E}+03$ & $<1.9 \mathrm{E}+03$ & $<2.0 \mathrm{E}+03$ & $<2.0 \mathrm{E}+03$ & - & $<1.9 \mathrm{E}+03$ & $2.81 \mathrm{E}+03$ & $2.24 \mathrm{E}+05$ & - \\
\hline $\mathrm{Sn}$ (IE) & $<1.3 \mathrm{E}+03$ & $<1.2 \mathrm{E}+03$ & $<1.3 \mathrm{E}+03$ & $<1.2 \mathrm{E}+03$ & - & $<1.2 \mathrm{E}+03$ & $<1.1 \mathrm{E}+03$ & - & - \\
\hline $\mathrm{Sr}$ (IE) & $8.67 \mathrm{E}+02$ & $7.56 \mathrm{E}+02$ & $8.17 \mathrm{E}+02$ & $8.13 \mathrm{E}+02$ & $6.8 \%$ & $<4.0 \mathrm{E}+01$ & $1.90 \mathrm{E}+03$ & $3.00 \mathrm{E}+01$ & $\mathrm{U}_{\mathrm{G}}$ \\
\hline Ti (IE) & $4.88 \mathrm{E}+01$ & $4.12 \mathrm{E}+01$ & $4.71 \mathrm{E}+01$ & $4.57 \mathrm{E}+01$ & $8.7 \%$ & $<1.2 \mathrm{E}+01$ & $5.74 \mathrm{E}+03$ & $6.90 \mathrm{E}+03$ & $\mathrm{U}_{\mathrm{G}} \mathrm{U}_{\mathrm{E}}$ \\
\hline U (IE) & $9.13 \mathrm{E}+03$ & $8.16 \mathrm{E}+03$ & $8.67 \mathrm{E}+03$ & $8.65 \mathrm{E}+03$ & $5.6 \%$ & $<7.8 \mathrm{E}+02$ & $<7.3 \mathrm{E}+02$ & - & - \\
\hline $\mathrm{Zr}$ (IE) & $4.83 \mathrm{E}+02$ & $4.44 \mathrm{E}+02$ & $4.71 \mathrm{E}+02$ & $4.66 \mathrm{E}+02$ & $4.3 \%$ & $<3.2 \mathrm{E}+01$ & $5.59 \mathrm{E}+02$ & $9.60 \mathrm{E}+02$ & $\mathrm{U}_{\mathrm{G}}$ \\
\hline $\mathrm{Hg}(\mathrm{CV})$ & $1.35 \mathrm{E}+04$ & $1.32 \mathrm{E}+04$ & $1.35 \mathrm{E}+04$ & $1.34 \mathrm{E}+04$ & $1.4 \%$ & - & - & - & - \\
\hline
\end{tabular}

* Single Value Divide $\mathrm{mg} / \mathrm{Kg}$ values by $1 \mathrm{E}+04$ to convert to $\mathrm{wt} \%$ basis

Data Quality Flags: $\quad U_{\mathrm{E}}-$ result is less than $10 \mathrm{X}$ the detection limit

$\mathrm{U}_{\mathrm{R}}-\% \mathrm{RSD}$ greater than $15 \%$

$\mathrm{U}_{\mathrm{E}}-$ result is less than $10 \mathrm{X}$ the detection limit
$\mathrm{U}_{\mathrm{B}}$ - blank result greater than $5 \%$ of sample result

$\mathrm{U}_{\mathrm{G}}$ - glass std result greater than $15 \%$ from expected result 
WSRC-TR-2005-00525, REV. 0

Table 3-5. Composition of the $1^{\text {st }}$ Aqua Regia Dissolution of Solids from Tank 51 Sample HTF-076-81 (continued)

\begin{tabular}{|c|c|c|c|c|c|c|c|}
\hline Analyte (Method) & $\begin{array}{c}\text { 1st } \\
\text { Replicate } \\
(\mathrm{mg} / \mathrm{Kg})\end{array}$ & $\begin{array}{c}\text { 2nd } \\
\text { Replicate } \\
(\mathrm{mg} / \mathrm{Kg})\end{array}$ & $\begin{array}{c}\text { 3rd } \\
\text { Replicate } \\
(\mathrm{mg} / \mathrm{Kg})\end{array}$ & $\begin{array}{l}\text { Average } \\
(\mathbf{m g} / \mathbf{K g})\end{array}$ & \%RSD & $\begin{array}{c}\text { Blank } \\
(\mathrm{mg} / \mathrm{Kg})\end{array}$ & $\begin{array}{c}\text { Data } \\
\text { Quality } \\
\text { Flags }\end{array}$ \\
\hline Th (IM) & $3.37 \mathrm{E}+02$ & $3.28 \mathrm{E}+02$ & $3.20 \mathrm{E}+02$ & $3.28 \mathrm{E}+02$ & $2.6 \%$ & $<2.0 \mathrm{E}-01$ & - \\
\hline $\mathrm{Ru}$ (IM) & $2.04 \mathrm{E}+02$ & $1.95 \mathrm{E}+02$ & $1.97 \mathrm{E}+02$ & $1.99 \mathrm{E}+02$ & $2.3 \%$ & $<2.9 \mathrm{E}+01$ & $\mathrm{U}_{\mathrm{E}}$ \\
\hline Ag (IM) & $1.31 \mathrm{E}+01$ & $1.34 \mathrm{E}+01$ & $1.34 \mathrm{E}+01$ & $1.33 \mathrm{E}+01$ & $1.3 \%$ & $<5.8 \mathrm{E}+00$ & $\mathrm{U}_{\mathrm{E}}$ \\
\hline $\mathrm{Rh}$ (IM) & $4.52 \mathrm{E}+01$ & $4.35 \mathrm{E}+01$ & $4.32 \mathrm{E}+01$ & $4.40 \mathrm{E}+01$ & $2.4 \%$ & $<4.0 \mathrm{E}-01$ & - \\
\hline $\mathrm{Pd}$ (IM) & $7.74 \mathrm{E}+00$ & $7.23 \mathrm{E}+00$ & $7.40 \mathrm{E}+00$ & $7.46 \mathrm{E}+00$ & $3.5 \%$ & $<3.0 \mathrm{E}-01$ & - \\
\hline $\mathrm{Tc}^{99}$ (IM) & $9.55 \mathrm{E}+00$ & $9.12 \mathrm{E}+00$ & $8.68 \mathrm{E}+00$ & $9.12 \mathrm{E}+00$ & $4.8 \%$ & $<4.8 \mathrm{E}+00$ & - \\
\hline $\mathrm{Np}^{237}$ (IM) & $5.79 \mathrm{E}+00$ & $5.82 \mathrm{E}+00$ & $5.61 \mathrm{E}+00$ & $5.74 \mathrm{E}+00$ & $1.9 \%$ & $<4.0 \mathrm{E}-01$ & - \\
\hline $\mathrm{Pu}^{239}(\mathrm{IM})$ & $4.90 \mathrm{E}+01$ & $4.86 \mathrm{E}+01$ & $4.64 \mathrm{E}+01$ & $4.80 \mathrm{E}+01$ & $2.9 \%$ & $<4.0 \mathrm{E}-01$ & - \\
\hline $\mathrm{Pu}^{240}$ (IM) & $6.26 \mathrm{E}+00$ & $6.26 \mathrm{E}+00$ & $6.15 \mathrm{E}+00$ & $6.22 \mathrm{E}+00$ & $1.0 \%$ & $<4.0 \mathrm{E}-01$ & - \\
\hline $\mathrm{Am}^{241} / \mathrm{Pu}^{241}$ (IM) & $3.19 \mathrm{E}+00$ & $2.97 \mathrm{E}+00$ & $2.95 \mathrm{E}+00$ & $3.04 \mathrm{E}+00$ & $4.5 \%$ & $<2.0 \mathrm{E}-01$ & - \\
\hline $\mathrm{Pu}^{242}(\mathrm{IM})$ & $6.65 \mathrm{E}-01$ & $4.71 \mathrm{E}-01$ & $5.16 \mathrm{E}-01$ & $5.51 \mathrm{E}-01$ & $18 \%$ & $<4.0 \mathrm{E}-01$ & $\mathrm{U}_{\mathrm{R}} \mathrm{U}_{\mathrm{E}}$ \\
\hline $\mathrm{Am}^{243}$ (IM) & $7.95 \mathrm{E}-01$ & $7.66 \mathrm{E}-01$ & $9.57 \mathrm{E}-01$ & $8.39 \mathrm{E}-01$ & $12 \%$ & $<2.0 \mathrm{E}-01$ & - \\
\hline $\mathrm{Cm}^{244}$ (IM) & $<2.1 \mathrm{E}-01$ & $2.60 \mathrm{E}-01$ & $<2.1 \mathrm{E}-01$ & $2.60 \mathrm{E}-01^{*}$ & - & $<2.0 \mathrm{E}-01$ & - \\
\hline $\mathrm{U}^{233}$ (IM) & $9.71 \mathrm{E}-01$ & 7.94E-01 & $9.11 \mathrm{E}-01$ & $8.92 \mathrm{E}-01$ & $10 \%$ & $<4.0 \mathrm{E}-01$ & $\mathrm{U}_{\mathrm{E}}$ \\
\hline $\mathrm{U}^{234}$ (IM) & $3.92 \mathrm{E}+00$ & $3.73 \mathrm{E}+00$ & $3.59 \mathrm{E}+00$ & $3.74 \mathrm{E}+00$ & $4.6 \%$ & $<2.0 \mathrm{E}-01$ & - \\
\hline $\mathrm{U}^{235}$ (IM) & $7.14 \mathrm{E}+01$ & $6.72 \mathrm{E}+01$ & $6.58 \mathrm{E}+01$ & $6.81 \mathrm{E}+01$ & $4.3 \%$ & $<2.0 \mathrm{E}-01$ & - \\
\hline $\mathrm{U}^{236}$ (IM) & $5.87 \mathrm{E}+00$ & $5.14 \mathrm{E}+00$ & $5.28 \mathrm{E}+00$ & $5.43 \mathrm{E}+00$ & $7.1 \%$ & $<4.0 \mathrm{E}-01$ & - \\
\hline $\mathrm{U}^{238}$ (IM) & $8.14 \mathrm{E}+03$ & $8.08 \mathrm{E}+03$ & $7.95 \mathrm{E}+03$ & $8.06 \mathrm{E}+03$ & $1.2 \%$ & $<6.0 \mathrm{E}-01$ & - \\
\hline U-total (sum) & $8.22 \mathrm{E}+03$ & $8.15 \mathrm{E}+03$ & $8.03 \mathrm{E}+03$ & $8.13 \mathrm{E}+03$ & $1.2 \%$ & - & - \\
\hline
\end{tabular}

* Single Value

Data Quality Flags:

$\mathrm{U}_{\mathrm{E}}-$ result is less than $10 \mathrm{X}$ the detection limit

$\mathrm{U}_{\mathrm{R}}-\% \mathrm{RSD}$ greater than $15 \%$

$\mathrm{U}_{\mathrm{B}}$ - blank result greater than $5 \%$ of sample result

$\mathrm{U}_{\mathrm{G}}-$ glass std result greater than $15 \%$ from expected result 
WSRC-TR-2005-00525, REV. 0

Table 3-5. Composition of the $1^{\text {st }}$ Aqua Regia Dissolution of Solids from Tank 51 Sample HTF-076-81 (continued)

\begin{tabular}{|l|c|c|c|c|c|c|c|}
\hline Analyte (Method) & $\begin{array}{c}\text { 1st } \\
\text { Replicate } \\
(\mathbf{m C i} / \mathbf{K g})\end{array}$ & $\begin{array}{c}\text { 2nd } \\
\text { Replicate } \\
\text { (mCi/Kg) }\end{array}$ & $\begin{array}{c}\text { 3rd } \\
\text { Replicate } \\
\text { (mCi/Kg) }\end{array}$ & $\begin{array}{c}\text { Average } \\
(\mathbf{m C i} / \mathbf{K g})\end{array}$ & \%RSD & $\begin{array}{c}\text { Data } \\
\text { (mCi/Kg) }\end{array}$ & $\begin{array}{c}\text { Quality } \\
\text { Flags }\end{array}$ \\
\hline $\mathrm{Cs}^{137}(\mathrm{GS})$ & $3.58 \mathrm{E}+02$ & $3.48 \mathrm{E}+02$ & $3.36 \mathrm{E}+02$ & $3.47 \mathrm{E}+02$ & $3.2 \%$ & $<1.0 \mathrm{E}+00$ & - \\
\hline $\mathrm{Cs}^{134}(\mathrm{GS})$ & $<1.5 \mathrm{E}+00$ & $<1.6 \mathrm{E}+00$ & $<1.4 \mathrm{E}+00$ & $<1.5 \mathrm{E}+00$ & - & $<8.7 \mathrm{E}-01$ & - \\
\hline $\mathrm{Eu}^{154}(\mathrm{GS})$ & $1.65 \mathrm{E}+01$ & $1.33 \mathrm{E}+01$ & $1.41 \mathrm{E}+01$ & $1.46 \mathrm{E}+01$ & $11 \%$ & $<1.8 \mathrm{E}+00$ & $\mathrm{U}_{\mathrm{E}}$ \\
\hline $\mathrm{Eu}^{155}(\mathrm{GS})$ & $<5.9 \mathrm{E}+00$ & $<5.8 \mathrm{E}+00$ & $<6.0 \mathrm{E}+00$ & $<5.9 \mathrm{E}+00$ & - & $<6.2 \mathrm{E}+00$ & - \\
\hline $\mathrm{Co}^{60}(\mathrm{GS})$ & $<1.2 \mathrm{E}+00$ & $<1.3 \mathrm{E}+00$ & $<1.3 \mathrm{E}+00$ & $<1.3 \mathrm{E}+00$ & - & $<1.1 \mathrm{E}+00$ & - \\
\hline $\mathrm{Ru}^{106}(\mathrm{GS})$ & $<1.1 \mathrm{E}+01$ & $<1.1 \mathrm{E}+01$ & $<1.1 \mathrm{E}+01$ & $<1.1 \mathrm{E}+01$ & - & $<5.7 \mathrm{E}+00$ & - \\
\hline $\mathrm{Ce}^{144}(\mathrm{GS})$ & $<1.4 \mathrm{E}+01$ & $<1.3 \mathrm{E}+01$ & $<1.3 \mathrm{E}+01$ & $<1.3 \mathrm{E}+01$ & - & $<6.1 \mathrm{E}+00$ & - \\
\hline $\mathrm{Sb}^{125}(\mathrm{GS})$ & $<7.9 \mathrm{E}+00$ & $<7.6 \mathrm{E}+00$ & $<8.0 \mathrm{E}+00$ & $<7.8 \mathrm{E}+00$ & - & $<2.8 \mathrm{E}+00$ & - \\
\hline $\mathrm{Am}^{241}(\mathrm{GS})$ & $8.11 \mathrm{E}+00$ & $<8.6 \mathrm{E}+00$ & $7.68 \mathrm{E}+00$ & $7.89 \mathrm{E}+00$ & $3.9 \%$ & $<5.0 \mathrm{E}+00$ & - \\
\hline $\mathrm{Sr}^{90}(\mathrm{BS})$ & $5.26 \mathrm{E}+03$ & $5.03 \mathrm{E}+03$ & $4.19 \mathrm{E}+03$ & $4.82 \mathrm{E}+03$ & $12 \%$ & $<1.1 \mathrm{E}+00$ & - \\
\hline $\mathrm{Pu}^{238}(\mathrm{TTA})$ & $1.67 \mathrm{E}+02$ & $1.55 \mathrm{E}+02$ & $1.67 \mathrm{E}+02$ & $1.63 \mathrm{E}+02$ & $4.2 \%$ & $1.50 \mathrm{E}+02$ & $\mathrm{U}_{\mathrm{B}}$ \\
\hline $\mathrm{Pu}^{239 / 240}(\mathrm{TTA})$ & $4.54 \mathrm{E}+00$ & $3.84 \mathrm{E}+00$ & $4.65 \mathrm{E}+00$ & $4.34 \mathrm{E}+00$ & $10 \%$ & $4.81 \mathrm{E}+00$ & $\mathrm{U} \mathrm{B}$ \\
\hline $\mathrm{Pu}^{241}(\mathrm{TTA})$ & $6.36 \mathrm{E}+01$ & $5.64 \mathrm{E}+01$ & $6.34 \mathrm{E}+01$ & $6.12 \mathrm{E}+01$ & $6.7 \%$ & $<2.8 \mathrm{E}+00$ & - \\
\hline
\end{tabular}

Data Quality Flags:

$\mathrm{U}_{\mathrm{E}}$ - result is less than $10 \mathrm{X}$ the detection limit

$\mathrm{U}_{\mathrm{R}}-\% \mathrm{RSD}$ greater than $15 \%$

$\mathrm{U}_{\mathrm{B}}$ - blank result greater than $5 \%$ of sample result

$\mathrm{U}_{\mathrm{G}}$ - glass std result greater than $15 \%$ from expected result 
WSRC-TR-2005-00525, REV. 0

Table 3-6. Composition of the $1^{\text {st }}$ Sodium Peroxide Fusion Dissolution of Solids from Tank 51 Sample HTF-076-81

\begin{tabular}{|c|c|c|c|c|c|c|c|c|c|}
\hline Analyte (Method) & $\begin{array}{c}\text { 1st } \\
\text { Replicate } \\
(\mathrm{mg} / \mathrm{Kg})\end{array}$ & $\begin{array}{c}\text { 2nd } \\
\text { Replicate } \\
(\mathrm{mg} / \mathrm{Kg})\end{array}$ & $\begin{array}{c}\text { 3rd } \\
\text { Replicate } \\
(\mathrm{mg} / \mathrm{Kg})\end{array}$ & $\begin{array}{l}\text { Average } \\
\text { (mg/Kg) }\end{array}$ & $\%$ RSD & $\begin{array}{c}\text { Blank } \\
(\mathrm{mg} / \mathrm{Kg})\end{array}$ & $\begin{array}{c}\text { Analyzed } \\
\text { Glass Std } \\
(\mathrm{mg} / \mathrm{Kg})\end{array}$ & $\begin{array}{c}\text { Glass Std } \\
\text { Composition } \\
(\mathrm{mg} / \mathrm{Kg})\end{array}$ & $\begin{array}{c}\text { Data } \\
\text { Quality } \\
\text { Flags } \\
\end{array}$ \\
\hline $\mathrm{Ag}$ (IE) & $2.31 \mathrm{E}+02$ & $2.86 \mathrm{E}+02$ & $3.27 \mathrm{E}+02$ & $2.81 \mathrm{E}+02$ & $17 \%$ & $3.67 \mathrm{E}+02$ & $2.23 \mathrm{E}+02$ & - & $\mathrm{U}_{\mathrm{B}} \mathrm{U}_{\mathrm{E}} \mathrm{U}_{\mathrm{R}}$ \\
\hline Al (IE) & $6.72 \mathrm{E}+04$ & $7.10 \mathrm{E}+04$ & $6.72 \mathrm{E}+04$ & $6.84 \mathrm{E}+04$ & $3.2 \%$ & $<1.7 \mathrm{E}+02$ & $2.17 \mathrm{E}+04$ & $2.50 \mathrm{E}+04$ & - \\
\hline B (IE) & $<2.1 \mathrm{E}+02$ & $<2.2 \mathrm{E}+02$ & $<2.1 \mathrm{E}+02$ & $<2.1 \mathrm{E}+02$ & - & $<2.1 \mathrm{E}+02$ & $2.36 \mathrm{E}+04$ & $2.69 \mathrm{E}+04$ & - \\
\hline $\mathrm{Ba}$ (IE) & $2.53 \mathrm{E}+02$ & $2.71 \mathrm{E}+02$ & $2.57 \mathrm{E}+02$ & $2.60 \mathrm{E}+02$ & $3.6 \%$ & $<9.2 \mathrm{E}+01$ & $8.33 \mathrm{E}+02$ & $7.90 \mathrm{E}+02$ & $\mathrm{U}_{\mathrm{E}}$ \\
\hline $\mathrm{Be}$ (IE) & $<9.7 \mathrm{E}+00$ & $<1.0 \mathrm{E}+01$ & $<9.4 \mathrm{E}+00$ & $<9.7 \mathrm{E}+00$ & - & $<9.6 \mathrm{E}+00$ & $2.47 \mathrm{E}+01$ & - & - \\
\hline $\mathrm{Ca}$ (IE) & $4.90 \mathrm{E}+03$ & $5.39 \mathrm{E}+03$ & $5.47 \mathrm{E}+03$ & $5.25 \mathrm{E}+03$ & $5.9 \%$ & $2.02 \mathrm{E}+03$ & $1.09 \mathrm{E}+04$ & $1.02 \mathrm{E}+04$ & $\mathrm{U}_{\mathrm{B}}$ \\
\hline $\mathrm{Cd}$ (IE) & $8.50 \mathrm{E}+01$ & $9.63 \mathrm{E}+01$ & $8.71 \mathrm{E}+01$ & $8.95 \mathrm{E}+01$ & $6.7 \%$ & $<1.7 \mathrm{E}+01$ & $<1.4 \mathrm{E}+01$ & - & $\mathrm{U}_{\mathrm{E}}$ \\
\hline $\mathrm{Ce}$ (IE) & $1.71 \mathrm{E}+03$ & $1.78 \mathrm{E}+03$ & $1.70 \mathrm{E}+03$ & $1.73 \mathrm{E}+03$ & $2.3 \%$ & $1.38 \mathrm{E}+03$ & $1.19 \mathrm{E}+03$ & - & $\mathrm{U}_{\mathrm{B}} \mathrm{U}_{\mathrm{E}}$ \\
\hline $\mathrm{Cr}$ (IE) & $7.21 \mathrm{E}+02$ & $2.49 \mathrm{E}+03$ & $2.62 \mathrm{E}+03$ & $1.94 \mathrm{E}+03$ & $55 \%$ & $<1.0 \mathrm{E}+02$ & $1.16 \mathrm{E}+03$ & $6.40 \mathrm{E}+02$ & $\mathrm{U}_{\mathrm{G}} \mathrm{U}_{\mathrm{R}}$ \\
\hline $\mathrm{Cu}$ (IE) & $8.99 \mathrm{E}+01$ & $1.03 \mathrm{E}+02$ & $9.73 \mathrm{E}+01$ & $9.68 \mathrm{E}+01$ & $7.0 \%$ & $<4.0 \mathrm{E}+01$ & $<3.3 \mathrm{E}+01$ & $3.00 \mathrm{E}+01$ & $\mathrm{U}_{\mathrm{E}}$ \\
\hline Gd (IE) & $1.44 \mathrm{E}+02$ & $1.23 \mathrm{E}+02$ & $1.28 \mathrm{E}+02$ & $1.32 \mathrm{E}+02$ & $8.3 \%$ & $<6.8 \mathrm{E}+01$ & $1.02 \mathrm{E}+02$ & - & $\mathrm{U}_{\mathrm{E}}$ \\
\hline K (IE) & $5.71 \mathrm{E}+03$ & $7.88 \mathrm{E}+03$ & $1.01 \mathrm{E}+04$ & $7.89 \mathrm{E}+03$ & $28 \%$ & $8.20 \mathrm{E}+03$ & $3.18 \mathrm{E}+04$ & $2.26 \mathrm{E}+04$ & $\mathrm{U}_{\mathrm{B}} \mathrm{U}_{\mathrm{G}} \mathrm{U}_{\mathrm{R}} \mathrm{U}_{\mathrm{E}}$ \\
\hline La (IE) & $3.17 \mathrm{E}+02$ & $3.49 \mathrm{E}+02$ & $3.34 \mathrm{E}+02$ & $3.33 \mathrm{E}+02$ & $4.8 \%$ & $<2.1 \mathrm{E}+02$ & $<1.7 \mathrm{E}+02$ & - & $\mathrm{U}_{\mathrm{E}}$ \\
\hline Li (IE) & $<1.9 \mathrm{E}+02$ & $<2.0 \mathrm{E}+02$ & $<1.9 \mathrm{E}+02$ & $<1.9 \mathrm{E}+02$ & - & $<1.9 \mathrm{E}+02$ & $1.43 \mathrm{E}+04$ & $1.49 \mathrm{E}+04$ & - \\
\hline $\mathrm{Mg}$ (IE) & $1.28 \mathrm{E}+03$ & $1.46 \mathrm{E}+03$ & $1.38 \mathrm{E}+03$ & $1.38 \mathrm{E}+03$ & $6.8 \%$ & $<1.6 \mathrm{E}+01$ & $4.78 \mathrm{E}+03$ & $5.20 \mathrm{E}+03$ & - \\
\hline $\mathrm{Mn}$ (IE) & $8.02 \mathrm{E}+03$ & $8.59 \mathrm{E}+03$ & $8.05 \mathrm{E}+03$ & $8.22 \mathrm{E}+03$ & $3.9 \%$ & $<8.0 \mathrm{E}+00$ & $1.39 \mathrm{E}+04$ & $1.46 \mathrm{E}+04$ & - \\
\hline Mo (IE) & $<5.4 \mathrm{E}+02$ & $<6.0 \mathrm{E}+02$ & $<5.2 \mathrm{E}+02$ & $<5.5 \mathrm{E}+02$ & - & $<5.3 \mathrm{E}+02$ & $4.52 \mathrm{E}+02$ & - & - \\
\hline
\end{tabular}

Divide $\mathrm{mg} / \mathrm{Kg}$ values by $1 \mathrm{E}+04$ to convert to $\mathrm{wt} \%$ basis

Data Quality Flags: $\quad U_{\mathrm{E}}$ - result is less than $10 \mathrm{X}$ the detection limit

$\mathrm{U}_{\mathrm{B}}$ - blank result greater than $5 \%$ of sample result

$\mathrm{U}_{\mathrm{R}}-\% \mathrm{RSD}$ greater than $15 \%$

$\mathrm{U}_{\mathrm{G}}-$ glass std result greater than $15 \%$ from expected result 
WSRC-TR-2005-00525, REV. 0

Table 3-6. Composition of the $1^{\text {st }}$ Sodium Peroxide Fusion Dissolution of Solids from Tank 51 Sample HTF-076-81 (continued)

\begin{tabular}{|c|c|c|c|c|c|c|c|c|c|}
\hline Analyte (Method) & $\begin{array}{c}\text { 1st } \\
\text { Replicate } \\
(\mathrm{mg} / \mathrm{Kg})\end{array}$ & $\begin{array}{c}\text { 2nd } \\
\text { Replicate } \\
(\mathrm{mg} / \mathrm{Kg})\end{array}$ & $\begin{array}{c}\text { 3rd } \\
\text { Replicate } \\
(\mathrm{mg} / \mathrm{Kg})\end{array}$ & $\begin{array}{l}\text { Average } \\
\text { (mg/Kg) }\end{array}$ & $\%$ RSD & $\begin{array}{c}\text { Blank } \\
(\mathrm{mg} / \mathrm{Kg})\end{array}$ & $\begin{array}{c}\text { Analyzed } \\
\text { Glass Std } \\
(\mathrm{mg} / \mathrm{Kg}) \\
\end{array}$ & $\begin{array}{c}\text { Glass Std } \\
\text { Composition } \\
(\mathrm{mg} / \mathrm{Kg})\end{array}$ & $\begin{array}{c}\text { Data } \\
\text { Quality } \\
\text { Flags } \\
\end{array}$ \\
\hline $\mathrm{Ni}$ (IE) & $2.86 \mathrm{E}+03$ & $3.68 \mathrm{E}+03$ & $3.34 \mathrm{E}+03$ & $3.29 \mathrm{E}+03$ & $12 \%$ & $<1.6 \mathrm{E}+02$ & $7.96 \mathrm{E}+03$ & $8.27 \mathrm{E}+03$ & - \\
\hline $\mathrm{P}$ (IE) & $<1.1 \mathrm{E}+03$ & $<1.1 \mathrm{E}+03$ & $<1.0 \mathrm{E}+03$ & $<1.1 \mathrm{E}+03$ & - & $<1.1 \mathrm{E}+03$ & $<8.9 \mathrm{E}+02$ & $1.10 \mathrm{E}+03$ & $\mathrm{U}_{\mathrm{G}}$ \\
\hline $\mathrm{Pb}$ (IE) & $<3.1 \mathrm{E}+03$ & $<3.2 \mathrm{E}+03$ & $<3.0 \mathrm{E}+03$ & $<3.1 \mathrm{E}+03$ & - & $<3.0 \mathrm{E}+03$ & $<2.5 \mathrm{E}+03$ & - & - \\
\hline S (IE) & $1.08 \mathrm{E}+04$ & $1.15 \mathrm{E}+04$ & $1.13 \mathrm{E}+04$ & $1.12 \mathrm{E}+04$ & $3.1 \%$ & $4.76 \mathrm{E}+02$ & $<2.9 \mathrm{E}+02$ & - & - \\
\hline $\mathrm{Sb}$ (IE) & $5.18 \mathrm{E}+02$ & $4.90 \mathrm{E}+02$ & $4.77 \mathrm{E}+02$ & $4.95 \mathrm{E}+02$ & $4.3 \%$ & $<4.6 \mathrm{E}+02$ & $5.62 \mathrm{E}+02$ & - & $\mathrm{U}_{\mathrm{E}}$ \\
\hline Si (IE) & $<2.0 \mathrm{E}+03$ & $<2.0 \mathrm{E}+03$ & $<1.9 \mathrm{E}+03$ & $<2.0 \mathrm{E}+03$ & - & $<1.9 \mathrm{E}+03$ & $2.05 \mathrm{E}+05$ & $2.24 \mathrm{E}+05$ & - \\
\hline $\mathrm{Sn}$ (IE) & $<1.2 \mathrm{E}+03$ & $1.28 \mathrm{E}+03$ & $1.22 \mathrm{E}+03$ & $1.25 \mathrm{E}+03 * *$ & $3.1 \%$ & $<1.2 \mathrm{E}+03$ & $2.05 \mathrm{E}+03$ & - & $\mathrm{U}_{\mathrm{E}}$ \\
\hline Sr (IE) & $1.15 \mathrm{E}+03$ & $1.26 \mathrm{E}+03$ & $1.26 \mathrm{E}+03$ & $1.22 \mathrm{E}+03$ & $5.2 \%$ & $5.16 \mathrm{E}+02$ & $2.24 \mathrm{E}+03$ & $3.00 \mathrm{E}+01$ & $\mathrm{U}_{\mathrm{B}} \mathrm{U}_{\mathrm{G}}$ \\
\hline Ti (IE) & $5.10 \mathrm{E}+01$ & $6.56 \mathrm{E}+01$ & $5.16 \mathrm{E}+01$ & $5.60 \mathrm{E}+01$ & $15 \%$ & $<1.2 \mathrm{E}+01$ & $6.72 \mathrm{E}+03$ & $6.90 \mathrm{E}+03$ & $\mathrm{U}_{\mathrm{E}}$ \\
\hline U (IE) & $9.27 \mathrm{E}+03$ & $9.79 \mathrm{E}+03$ & $9.57 \mathrm{E}+03$ & $9.54 \mathrm{E}+03$ & $2.7 \%$ & $<7.8 \mathrm{E}+02$ & $6.96 \mathrm{E}+02$ & - & - \\
\hline $\mathrm{Zn}$ (IE) & $1.25 \mathrm{E}+02$ & $1.99 \mathrm{E}+02$ & $2.00 \mathrm{E}+02$ & $1.75 \mathrm{E}+02$ & $25 \%$ & $<2.8 \mathrm{E}+01$ & $1.93 \mathrm{E}+02$ & $1.60 \mathrm{E}+02$ & $\mathrm{U}_{\mathrm{G}} \mathrm{U}_{\mathrm{R}} \mathrm{U}_{\mathrm{E}}$ \\
\hline $\mathrm{Zr}$ (IE) & $3.66 \mathrm{E}+04$ & $5.06 \mathrm{E}+04$ & $5.90 \mathrm{E}+04$ & $4.87 \mathrm{E}+04$ & $23 \%$ & $7.32 \mathrm{E}+04$ & $4.15 E+04$ & $9.60 \mathrm{E}+02$ & $\mathrm{U}_{\mathrm{B}} \mathrm{U}_{\mathrm{R}} \mathrm{U}_{\mathrm{G}}$ \\
\hline
\end{tabular}

* Single Value $\quad * *$ Average of two replicates

Data Quality Flags: $\quad \mathrm{U}_{\mathrm{E}}-$ result is less than $10 \mathrm{X}$ the detection limit

$\mathrm{U}_{\mathrm{B}}$ - blank result greater than $5 \%$ of sample result
Divide $\mathrm{mg} / \mathrm{Kg}$ values by $1 \mathrm{E}+04$ to convert to $\mathrm{wt} \%$ basis $\mathrm{U}_{\mathrm{R}}-\% \mathrm{RSD}$ greater than $15 \%$

$\mathrm{U}_{\mathrm{G}}-$ glass std result greater than $15 \%$ from expected result 
WSRC-TR-2005-00525, REV. 0

Table 3-7. Composition of the $2^{\text {nd }}$ Aqua Regia Dissolution of Solids from Tank 51 Sample HTF-076-81

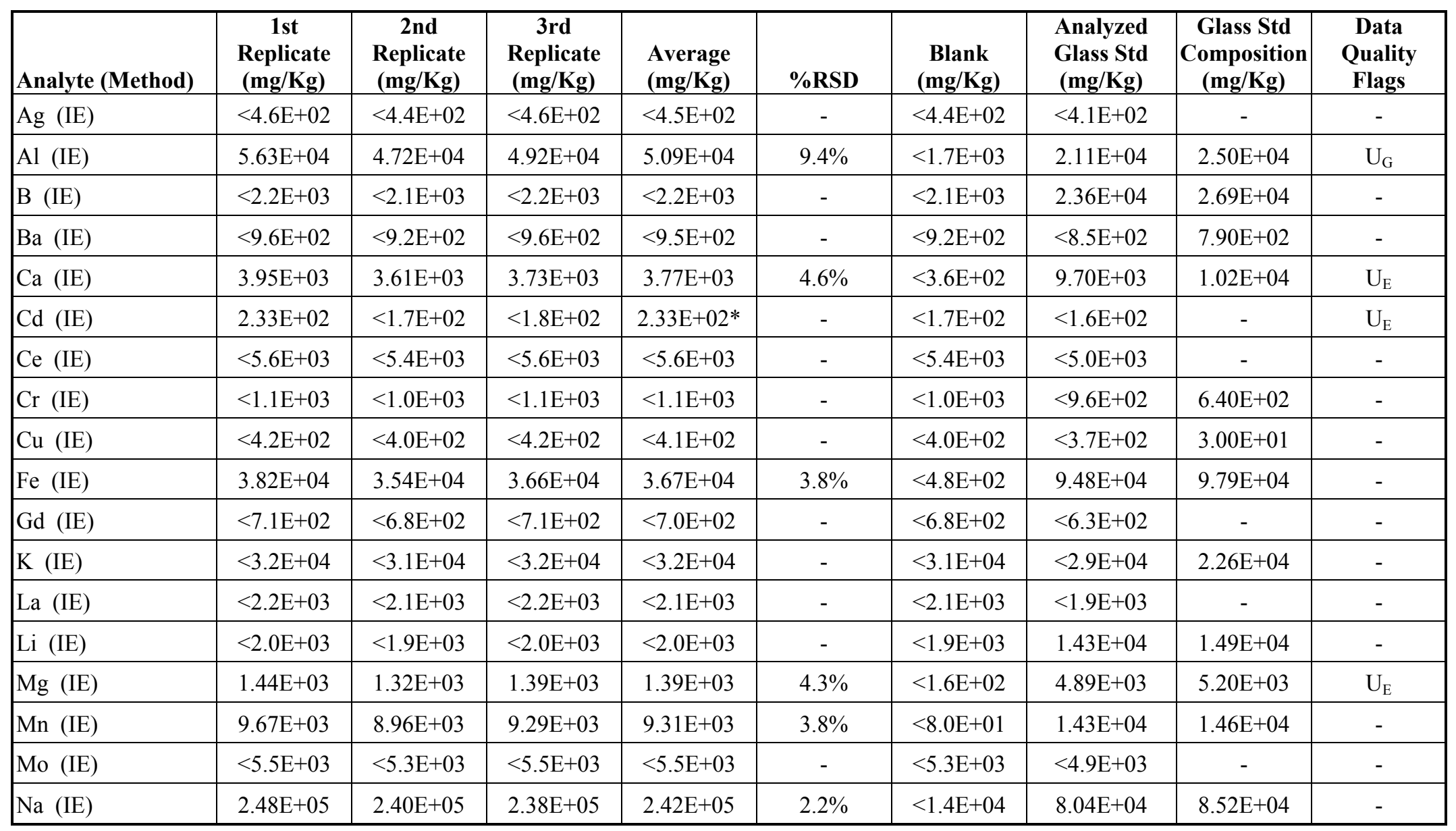

* Single Value Divide $\mathrm{mg} / \mathrm{Kg}$ values by $1 \mathrm{E}+04$ to convert to $\mathrm{wt} \%$ basis

Data Quality Flags: $\quad \mathrm{U}_{\mathrm{E}}$ - result is less than 10X the detection limit

$\mathrm{U}_{\mathrm{R}}-\% \mathrm{RSD}$ greater than $15 \%$

$U_{B}$ - blank result greater than $5 \%$ of sample result $\quad U_{G}-$ glass std result greater than $15 \%$ from expected result 
WSRC-TR-2005-00525, REV. 0

Table 3-7. Composition of the $2^{\text {nd }}$ Aqua Regia Dissolution of Solids from Tank 51 Sample HTF-076-81 (continued)

\begin{tabular}{|c|c|c|c|c|c|c|c|c|c|}
\hline Analyte (Method) & $\begin{array}{c}\text { 1st } \\
\text { Replicate } \\
(\mathrm{mg} / \mathrm{Kg})\end{array}$ & $\begin{array}{c}\text { 2nd } \\
\text { Replicate } \\
(\mathrm{mg} / \mathrm{Kg})\end{array}$ & $\begin{array}{c}\text { 3rd } \\
\text { Replicate } \\
(\mathrm{mg} / \mathrm{Kg})\end{array}$ & $\begin{array}{l}\text { Average } \\
\text { (mg/Kg) }\end{array}$ & \%RSD & $\begin{array}{c}\text { Blank } \\
\text { (mg/Kg) }\end{array}$ & $\begin{array}{c}\text { Analyzed } \\
\text { Glass Std } \\
(\mathbf{m g} / \mathrm{Kg})\end{array}$ & $\begin{array}{c}\text { Glass Std } \\
\text { Composition } \\
(\mathrm{mg} / \mathrm{Kg})\end{array}$ & $\begin{array}{c}\text { Data } \\
\text { Quality } \\
\text { Flags } \\
\end{array}$ \\
\hline $\mathrm{Ni}$ (IE) & $2.83 \mathrm{E}+03$ & $2.97 \mathrm{E}+03$ & $3.07 \mathrm{E}+03$ & $2.96 \mathrm{E}+03$ & $4.0 \%$ & $<1.6 \mathrm{E}+03$ & $7.33 \mathrm{E}+03$ & $8.27 \mathrm{E}+03$ & $\mathrm{U}_{\mathrm{E}}$ \\
\hline $\mathrm{P}$ (IE) & $<1.1 \mathrm{E}+04$ & $<1.1 \mathrm{E}+04$ & $<1.1 \mathrm{E}+04$ & $<1.1 \mathrm{E}+04$ & - & $<1.1 \mathrm{E}+04$ & $<9.9 \mathrm{E}+03$ & $1.10 \mathrm{E}+03$ & - \\
\hline $\mathrm{Pb}$ (IE) & $<3.2 \mathrm{E}+04$ & $<3.0 \mathrm{E}+04$ & $<3.2 \mathrm{E}+04$ & $<3.1 \mathrm{E}+04$ & - & $<3.0 \mathrm{E}+04$ & $<2.8 \mathrm{E}+04$ & - & - \\
\hline $\mathrm{S}$ (IE) & $7.63 \mathrm{E}+03$ & $7.56 \mathrm{E}+03$ & $1.02 \mathrm{E}+04$ & $8.45 \mathrm{E}+03$ & $18 \%$ & $<3.4 \mathrm{E}+03$ & $<3.2 \mathrm{E}+03$ & - & $\mathrm{U}_{\mathrm{E}} \mathrm{U}_{\mathrm{R}}$ \\
\hline $\mathrm{Sb}$ (IE) & $<4.8 \mathrm{E}+03$ & $<4.6 \mathrm{E}+03$ & $<4.8 \mathrm{E}+03$ & $<4.8 \mathrm{E}+03$ & - & $<4.6 \mathrm{E}+03$ & $<4.3 \mathrm{E}+03$ & - & - \\
\hline $\mathrm{Si}$ (IE) & $<2.0 \mathrm{E}+04$ & $<1.9 \mathrm{E}+04$ & $<2.0 \mathrm{E}+04$ & $<2.0 \mathrm{E}+04$ & - & $<1.9 \mathrm{E}+04$ & $<1.8 \mathrm{E}+04$ & $2.24 \mathrm{E}+05$ & - \\
\hline $\mathrm{Sn}$ (IE) & $<1.3 \mathrm{E}+04$ & $<1.2 \mathrm{E}+04$ & $<1.3 \mathrm{E}+04$ & $<1.2 \mathrm{E}+04$ & - & $<1.2 \mathrm{E}+04$ & $<1.1 \mathrm{E}+04$ & - & - \\
\hline $\mathrm{Sr}$ (IE) & $1.18 \mathrm{E}+03$ & $9.40 \mathrm{E}+02$ & $1.21 \mathrm{E}+03$ & $1.11 \mathrm{E}+03$ & $13 \%$ & $<4.0 \mathrm{E}+02$ & $2.16 \mathrm{E}+03$ & $3.00 \mathrm{E}+01$ & $\mathrm{U}_{\mathrm{G}} \mathrm{U}_{\mathrm{E}}$ \\
\hline Ti (IE) & $<1.3 \mathrm{E}+02$ & $<1.2 \mathrm{E}+02$ & $<1.3 \mathrm{E}+02$ & $<1.2 \mathrm{E}+02$ & - & $<1.2 \mathrm{E}+02$ & $5.74 \mathrm{E}+03$ & $6.90 \mathrm{E}+03$ & $\mathrm{U}_{\mathrm{G}}$ \\
\hline U (IE) & $<8.2 \mathrm{E}+03$ & $<7.8 \mathrm{E}+03$ & $<8.2 \mathrm{E}+03$ & $<8.1 \mathrm{E}+03$ & - & $<7.8 \mathrm{E}+03$ & $<7.3 \mathrm{E}+03$ & - & - \\
\hline V (IE) & $<8.3 \mathrm{E}+02$ & $<8.0 \mathrm{E}+02$ & $<8.3 \mathrm{E}+02$ & $<8.2 \mathrm{E}+02$ & - & $<8.0 \mathrm{E}+02$ & $<7.4 \mathrm{E}+02$ & - & - \\
\hline $\mathrm{Zn}$ (IE) & $<2.9 \mathrm{E}+02$ & $<2.8 \mathrm{E}+02$ & $<2.9 \mathrm{E}+02$ & $<2.9 \mathrm{E}+02$ & - & $<2.8 \mathrm{E}+02$ & $2.71 \mathrm{E}+02$ & $1.60 \mathrm{E}+02$ & $\mathrm{U}_{\mathrm{G}}$ \\
\hline $\mathrm{Zr}$ (IE) & $3.88 \mathrm{E}+02$ & $4.44 \mathrm{E}+02$ & $3.77 \mathrm{E}+02$ & $4.03 \mathrm{E}+02$ & $8.9 \%$ & $<3.2 \mathrm{E}+02$ & $4.81 \mathrm{E}+02$ & $9.60 \mathrm{E}+02$ & $\mathrm{U}_{\mathrm{G}} \mathrm{U}_{\mathrm{E}}$ \\
\hline
\end{tabular}

Divide $\mathrm{mg} / \mathrm{Kg}$ values by $1 \mathrm{E}+04$ to convert to $\mathrm{wt} \%$ basis

Data Quality Flags: $\quad \mathrm{U}_{\mathrm{E}}$ - result is less than $10 \mathrm{X}$ the detection limit

$\mathrm{U}_{\mathrm{B}}$ - blank result greater than $5 \%$ of sample result

$\mathrm{U}_{\mathrm{R}}-\% \mathrm{RSD}$ greater than $15 \%$

$\mathrm{U}_{\mathrm{G}}$ - glass std result greater than $15 \%$ from expected result 
WSRC-TR-2005-00525, REV. 0

Table 3-8. Composition of the $2^{\text {nd }}$ Sodium Peroxide Fusion Dissolution of Solids from Tank 51 Sample HTF-076-81

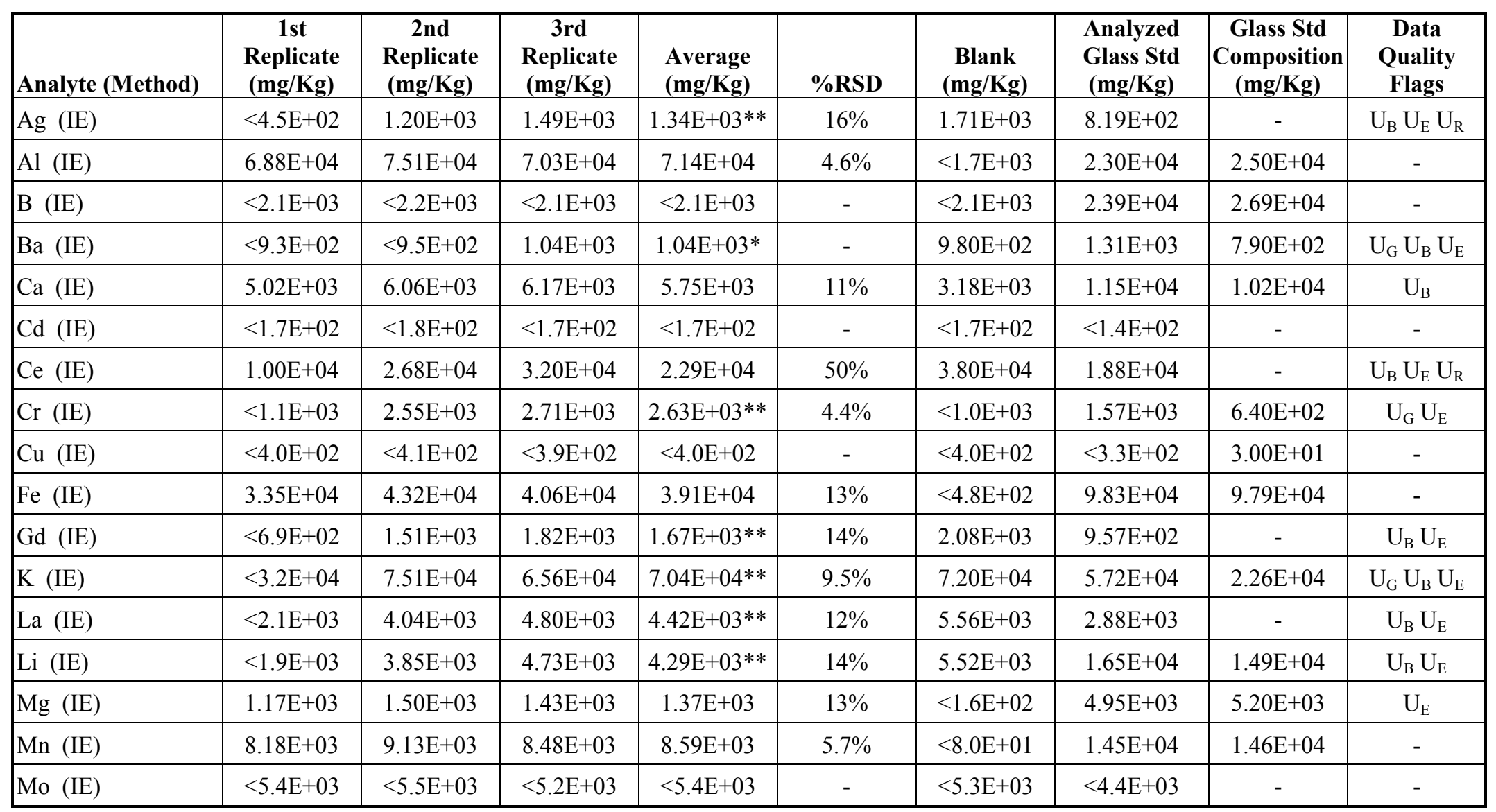

* Single Value

Data Quality Flags:
** Average of two replicates

$\mathrm{U}_{\mathrm{E}}$ - result is less than $10 \mathrm{X}$ the detection limit

$\mathrm{U}_{\mathrm{B}}$ - blank result greater than $5 \%$ of sample result
Divide $\mathrm{mg} / \mathrm{Kg}$ values by $1 \mathrm{E}+04$ to convert to $\mathrm{wt} \%$ basis

$\mathrm{U}_{\mathrm{R}}-\% \mathrm{RSD}$ greater than $15 \%$

$\mathrm{U}_{\mathrm{G}}-$ glass std result greater than $15 \%$ from expected result 
WSRC-TR-2005-00525, REV. 0

Table 3-8. Composition of the $2^{\text {nd }}$ Sodium Peroxide Fusion Dissolution of Solids from Tank 51 Sample HTF-076-81 (continued)

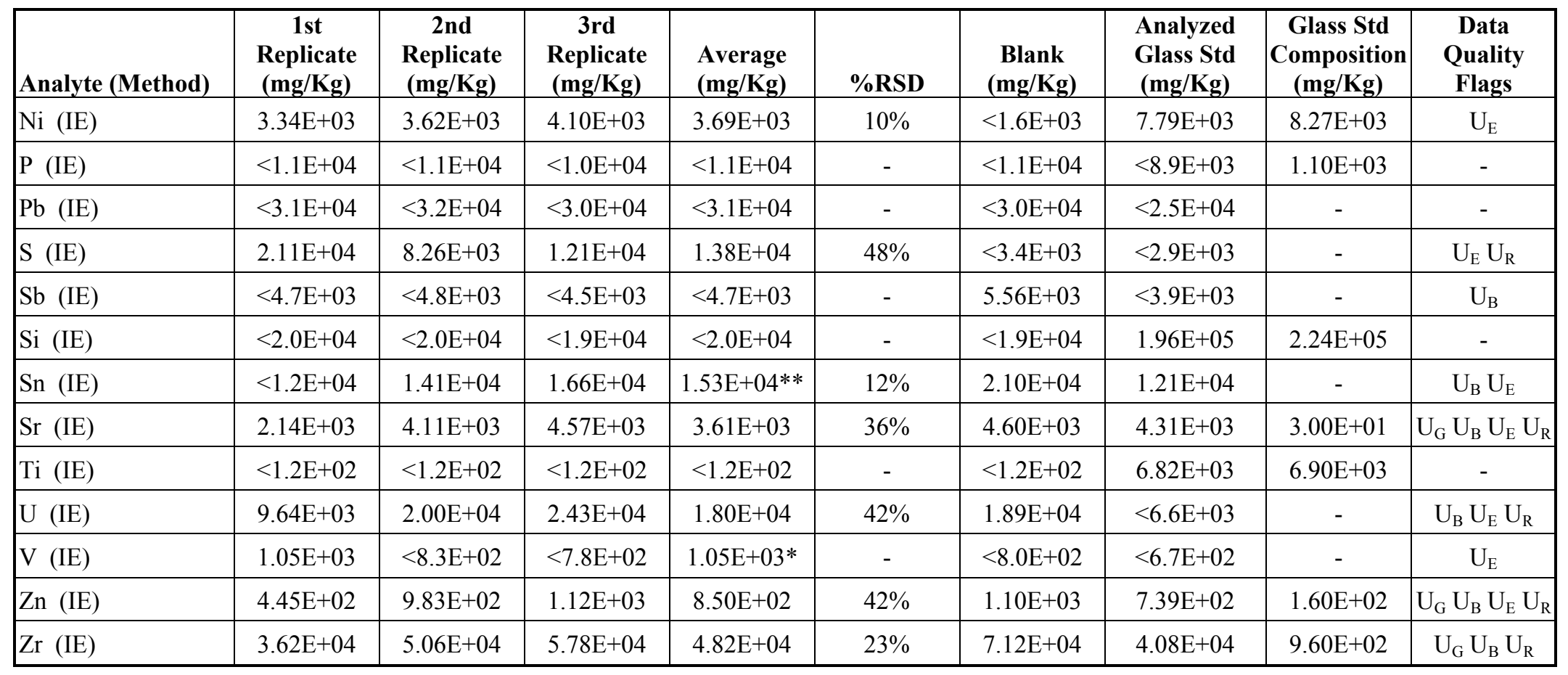

* Single Value $\quad * *$ Average of two replicates

Data Quality Flags: $\quad \mathrm{U}_{\mathrm{E}}-$ result is less than $10 \mathrm{X}$ the detection limit

$\mathrm{U}_{\mathrm{B}}$ - blank result greater than $5 \%$ of sample result
Divide $\mathrm{mg} / \mathrm{Kg}$ values by $1 \mathrm{E}+04$ to convert to $\mathrm{wt} \%$ basis $\mathrm{U}_{\mathrm{R}}-\% \mathrm{RSD}$ greater than $15 \%$

$\mathrm{U}_{\mathrm{G}}-$ glass std result greater than $15 \%$ from expected result 
WSRC-TR-2005-00525, REV. 0

Table 3-9. Composition of the $3^{\text {rd }}$ Aqua Regia Dissolution of Solids from Tank 51 Sample HTF-076-81

\begin{tabular}{|c|c|c|c|c|c|c|c|c|c|}
\hline Analyte (Method) & $\begin{array}{c}\text { 1st } \\
\text { Replicate } \\
(\mathrm{mg} / \mathrm{Kg})\end{array}$ & $\begin{array}{c}\text { 2nd } \\
\text { Replicate } \\
(\mathrm{mg} / \mathrm{Kg})\end{array}$ & $\begin{array}{c}\text { 3rd } \\
\text { Replicate } \\
(\mathrm{mg} / \mathrm{Kg}) \\
\end{array}$ & $\begin{array}{l}\text { Average } \\
\text { (mg/Kg) }\end{array}$ & $\%$ RSD & $\begin{array}{c}\text { Blank } \\
(\mathrm{mg} / \mathrm{Kg})\end{array}$ & $\begin{array}{c}\text { Analyzed } \\
\text { Glass Std } \\
(\mathrm{mg} / \mathrm{Kg}) \\
\end{array}$ & \begin{tabular}{|c|}
$\begin{array}{c}\text { Glass Std } \\
\text { Composition } \\
(\mathrm{mg} / \mathrm{Kg})\end{array}$ \\
\end{tabular} & $\begin{array}{c}\text { Data } \\
\text { Quality } \\
\text { Flags } \\
\end{array}$ \\
\hline $\mathrm{Ag}(\mathrm{IE})$ & $<7.3 \mathrm{E}+01$ & $<6.8 \mathrm{E}+01$ & $<6.7 \mathrm{E}+01$ & $<6.9 \mathrm{E}+01$ & - & $<7.6 \mathrm{E}+01$ & $<6.9 \mathrm{E}+01$ & - & - \\
\hline Al (IE) & $6.05 \mathrm{E}+04$ & $6.09 \mathrm{E}+04$ & $5.83 \mathrm{E}+04$ & $5.99 \mathrm{E}+04$ & $2.3 \%$ & $<4.6 \mathrm{E}+01$ & $2.19 \mathrm{E}+04$ & $2.50 \mathrm{E}+04$ & - \\
\hline B (IE) & $<5.5 \mathrm{E}+01$ & $<5.2 \mathrm{E}+01$ & $<5.1 \mathrm{E}+01$ & $<5.3 \mathrm{E}+01$ & - & $<5.8 \mathrm{E}+01$ & $2.65 \mathrm{E}+04$ & $2.69 \mathrm{E}+04$ & - \\
\hline $\mathrm{Ba}$ (IE) & $2.24 \mathrm{E}+02$ & $2.29 \mathrm{E}+02$ & $2.29 \mathrm{E}+02$ & $2.27 \mathrm{E}+02$ & $1.1 \%$ & $<1.2 \mathrm{E}+01$ & $8.02 \mathrm{E}+02$ & $7.90 \mathrm{E}+02$ & - \\
\hline $\mathrm{Ca}$ (IE) & $4.14 \mathrm{E}+03$ & $4.28 \mathrm{E}+03$ & $4.15 \mathrm{E}+03$ & $4.19 \mathrm{E}+03$ & $1.8 \%$ & $<1.0 \mathrm{E}+01$ & $1.00 \mathrm{E}+04$ & $1.02 \mathrm{E}+04$ & - \\
\hline $\mathrm{Cd}$ (IE) & $1.27 \mathrm{E}+02$ & $1.28 \mathrm{E}+02$ & $1.25 \mathrm{E}+02$ & $1.27 \mathrm{E}+02$ & $1.4 \%$ & $<1.7 \mathrm{E}+01$ & $<1.5 \mathrm{E}+01$ & - & $\mathrm{U}_{\mathrm{E}}$ \\
\hline $\mathrm{Ce}$ (IE) & $6.01 \mathrm{E}+02$ & $6.15 \mathrm{E}+02$ & $5.78 \mathrm{E}+02$ & $5.98 \mathrm{E}+02$ & $3.1 \%$ & $<1.5 \mathrm{E}+02$ & $2.60 \mathrm{E}+02$ & - & $\mathrm{U}_{\mathrm{E}}$ \\
\hline $\mathrm{Cr}$ (IE) & $4.08 \mathrm{E}+02$ & $4.64 \mathrm{E}+02$ & $3.91 \mathrm{E}+02$ & $4.21 \mathrm{E}+02$ & $9.0 \%$ & $<3.9 \mathrm{E}+01$ & $5.52 \mathrm{E}+02$ & $6.40 \mathrm{E}+02$ & - \\
\hline $\mathrm{Cu}$ (IE) & $1.38 \mathrm{E}+02$ & $1.41 \mathrm{E}+02$ & $1.37 \mathrm{E}+02$ & $1.39 \mathrm{E}+02$ & $1.3 \%$ & $<1.2 \mathrm{E}+01$ & $2.91 \mathrm{E}+01$ & $3.00 \mathrm{E}+01$ & - \\
\hline $\mathrm{Fe}$ (IE) & $3.63 \mathrm{E}+04$ & $3.75 E+04$ & $3.67 \mathrm{E}+04$ & $3.68 \mathrm{E}+04$ & $1.6 \%$ & $<1.2 \mathrm{E}+01$ & $9.64 \mathrm{E}+04$ & $9.79 \mathrm{E}+04$ & - \\
\hline K (IE) & $1.69 \mathrm{E}+03$ & $1.35 \mathrm{E}+03$ & $1.56 \mathrm{E}+03$ & $1.53 \mathrm{E}+03$ & $11 \%$ & $<8.6 \mathrm{E}+02$ & $2.32 \mathrm{E}+04$ & $2.26 \mathrm{E}+04$ & $\mathrm{U}_{\mathrm{E}}$ \\
\hline La (IE) & $2.13 \mathrm{E}+02$ & $2.18 \mathrm{E}+02$ & $2.12 \mathrm{E}+02$ & $2.14 \mathrm{E}+02$ & $1.5 \%$ & $<5.7 \mathrm{E}+01$ & $<5.2 \mathrm{E}+01$ & - & $\mathrm{U}_{\mathrm{E}}$ \\
\hline Li (IE) & $6.87 \mathrm{E}+01$ & $<4.7 \mathrm{E}+01$ & $<4.7 \mathrm{E}+01$ & $6.87 \mathrm{E}+01 *$ & - & $<5.3 \mathrm{E}+01$ & $1.47 \mathrm{E}+04$ & $1.49 \mathrm{E}+04$ & $\mathrm{U}_{\mathrm{E}}$ \\
\hline $\mathrm{Mg}$ (IE) & $1.53 \mathrm{E}+03$ & $1.57 \mathrm{E}+03$ & $1.55 \mathrm{E}+03$ & $1.55 \mathrm{E}+03$ & $1.6 \%$ & $<4.0 \mathrm{E}+00$ & $5.15 \mathrm{E}+03$ & $5.20 \mathrm{E}+03$ & - \\
\hline Mn (IE) & $9.01 \mathrm{E}+03$ & $9.25 \mathrm{E}+03$ & $9.10 \mathrm{E}+03$ & $9.12 \mathrm{E}+03$ & $1.4 \%$ & $<2.0 \mathrm{E}+00$ & $1.45 \mathrm{E}+04$ & $1.46 \mathrm{E}+04$ & - \\
\hline Mo (IE) & $1.97 \mathrm{E}+02$ & $<1.3 \mathrm{E}+02$ & $1.58 \mathrm{E}+02$ & $1.62 \mathrm{E}+02 * *$ & $21 \%$ & $<1.5 \mathrm{E}+02$ & $2.81 \mathrm{E}+02$ & - & $\mathrm{U}_{\mathrm{R}} \mathrm{U}_{\mathrm{E}}$ \\
\hline $\mathrm{Na}$ (IE) & $2.27 \mathrm{E}+05$ & $2.17 \mathrm{E}+05$ & $2.24 \mathrm{E}+05$ & $2.23 \mathrm{E}+05$ & $2.3 \%$ & $<3.9 \mathrm{E}+02$ & $8.22 \mathrm{E}+04$ & $8.52 \mathrm{E}+04$ & - \\
\hline
\end{tabular}

* Single Value $\quad * *$ Average of two replicates

Data Quality Flags: $\quad U_{\mathrm{E}}-$ result is less than $10 \mathrm{X}$ the detection limit

$\mathrm{U}_{\mathrm{B}}$ - blank result greater than $5 \%$ of sample result
Divide $\mathrm{mg} / \mathrm{Kg}$ values by $1 \mathrm{E}+04$ to convert to $\mathrm{wt} \%$ basis $\mathrm{U}_{\mathrm{R}}-\% \mathrm{RSD}$ greater than $15 \%$

$\mathrm{U}_{\mathrm{G}}-$ glass std result greater than $15 \%$ from expected result 
WSRC-TR-2005-00525, REV. 0

Table 3-9. Composition of the $3^{\text {rd }}$ Aqua Regia Dissolution of Solids from Tank 51 Sample HTF-076-81 (continued)

\begin{tabular}{|c|c|c|c|c|c|c|c|c|c|}
\hline Analyte (Method) & $\begin{array}{c}\text { 1st } \\
\text { Replicate } \\
(\mathrm{mg} / \mathrm{Kg})\end{array}$ & $\begin{array}{c}\text { 2nd } \\
\text { Replicate } \\
\text { (mg/Kg) }\end{array}$ & $\begin{array}{c}\text { 3rd } \\
\text { Replicate } \\
(\mathrm{mg} / \mathrm{Kg})\end{array}$ & $\begin{array}{l}\text { Average } \\
\text { (mg/Kg) }\end{array}$ & \%RSD & $\begin{array}{c}\text { Blank } \\
(\mathrm{mg} / \mathrm{Kg})\end{array}$ & $\begin{array}{c}\text { Analyzed } \\
\text { Glass Std } \\
(\mathbf{m g} / \mathrm{Kg})\end{array}$ & $\begin{array}{c}\text { Glass Std } \\
\text { Composition } \\
(\mathrm{mg} / \mathrm{Kg})\end{array}$ & $\begin{array}{c}\text { Data } \\
\text { Quality } \\
\text { Flags } \\
\end{array}$ \\
\hline $\mathrm{Ni}$ (IE) & $3.23 \mathrm{E}+03$ & $3.29 \mathrm{E}+03$ & $3.26 \mathrm{E}+03$ & $3.26 \mathrm{E}+03$ & $1.0 \%$ & $<4.1 \mathrm{E}+01$ & $8.40 \mathrm{E}+03$ & $8.27 \mathrm{E}+03$ & - \\
\hline $\mathrm{P}$ (IE) & $7.63 \mathrm{E}+02$ & $5.28 \mathrm{E}+02$ & $8.06 \mathrm{E}+02$ & $6.99 \mathrm{E}+02$ & $21 \%$ & $<2.9 \mathrm{E}+02$ & $1.07 \mathrm{E}+03$ & $1.10 \mathrm{E}+03$ & $\mathrm{U}_{\mathrm{R}} \mathrm{U}_{\mathrm{E}}$ \\
\hline $\mathrm{Pb}$ (IE) & $<2.7 \mathrm{E}+02$ & $<2.5 \mathrm{E}+02$ & $<2.5 \mathrm{E}+02$ & $<2.6 \mathrm{E}+02$ & - & $<2.9 \mathrm{E}+02$ & $<2.6 \mathrm{E}+02$ & - & - \\
\hline S (IE) & $8.79 \mathrm{E}+03$ & $7.86 \mathrm{E}+03$ & $9.19 \mathrm{E}+03$ & $8.61 \mathrm{E}+03$ & $7.9 \%$ & $<9.5 \mathrm{E}+01$ & $2.75 \mathrm{E}+02$ & - & - \\
\hline $\mathrm{Sb}$ (IE) & $2.97 \mathrm{E}+02$ & $2.85 \mathrm{E}+02$ & $2.88 \mathrm{E}+02$ & $2.90 \mathrm{E}+02$ & $2.2 \%$ & $<1.3 \mathrm{E}+02$ & $4.01 \mathrm{E}+02$ & - & $\mathrm{U}_{\mathrm{E}}$ \\
\hline $\mathrm{Si}$ (IE) & $1.11 \mathrm{E}+03$ & $8.51 \mathrm{E}+02$ & $1.05 \mathrm{E}+03$ & $1.00 \mathrm{E}+03$ & $13 \%$ & $<5.4 \mathrm{E}+02$ & $1.28 \mathrm{E}+04$ & $2.24 \mathrm{E}+05$ & $\mathrm{U}_{\mathrm{G}} \mathrm{U}_{\mathrm{E}}$ \\
\hline $\mathrm{Sn}$ (IE) & $<3.1 \mathrm{E}+02$ & $<2.9 \mathrm{E}+02$ & $<2.9 \mathrm{E}+02$ & $<3.0 \mathrm{E}+02$ & - & $<3.3 \mathrm{E}+02$ & $7.34 \mathrm{E}+02$ & - & - \\
\hline $\mathrm{Sr}$ (IE) & $9.00 \mathrm{E}+02$ & $9.25 \mathrm{E}+02$ & $9.01 \mathrm{E}+02$ & $9.09 \mathrm{E}+02$ & $1.6 \%$ & $<1.1 \mathrm{E}+01$ & $1.96 \mathrm{E}+03$ & $3.00 \mathrm{E}+01$ & $\mathrm{U}_{\mathrm{G}}$ \\
\hline Ti (IE) & $4.77 \mathrm{E}+01$ & $4.80 \mathrm{E}+01$ & $4.77 \mathrm{E}+01$ & $4.78 \mathrm{E}+01$ & $0.4 \%$ & $<3.0 \mathrm{E}+00$ & $5.99 \mathrm{E}+03$ & $6.90 \mathrm{E}+03$ & - \\
\hline U (IE) & $9.00 \mathrm{E}+03$ & $9.16 \mathrm{E}+03$ & $9.01 \mathrm{E}+03$ & $9.06 \mathrm{E}+03$ & $1.0 \%$ & $<2.2 \mathrm{E}+02$ & $3.27 \mathrm{E}+02$ & - & - \\
\hline $\mathrm{V}$ (IE) & $4.20 \mathrm{E}+01$ & $2.58 \mathrm{E}+01$ & $1.69 \mathrm{E}+02$ & $7.88 \mathrm{E}+01$ & $99 \%$ & $<2.2 \mathrm{E}+01$ & $9.64 \mathrm{E}+01$ & - & $\mathrm{U}_{\mathrm{R}} \mathrm{U}_{\mathrm{E}}$ \\
\hline $\mathrm{Zn}$ (IE) & $1.43 \mathrm{E}+02$ & $1.49 \mathrm{E}+02$ & $1.45 \mathrm{E}+02$ & $1.46 \mathrm{E}+02$ & $2.2 \%$ & $6.80 \mathrm{E}+01$ & $2.69 \mathrm{E}+02$ & $1.60 \mathrm{E}+02$ & $\mathrm{U}_{\mathrm{G}} \mathrm{U}_{\mathrm{B}}$ \\
\hline $\mathrm{Zr}$ (IE) & $5.02 \mathrm{E}+02$ & $2.58 \mathrm{E}+02$ & $4.98 \mathrm{E}+02$ & $4.19 \mathrm{E}+02$ & $33 \%$ & $<9.0 \mathrm{E}+00$ & $5.23 \mathrm{E}+02$ & $9.60 \mathrm{E}+02$ & $\mathrm{U}_{\mathrm{R}} \mathrm{U}_{\mathrm{G}}$ \\
\hline
\end{tabular}

Divide $\mathrm{mg} / \mathrm{Kg}$ values by $1 \mathrm{E}+04$ to convert to $\mathrm{wt} \%$ basis

Data Quality Flags: $\quad \mathrm{U}_{\mathrm{E}}-$ result is less than $10 \mathrm{X}$ the detection limit

$\mathrm{U}_{\mathrm{B}}$ - blank result greater than $5 \%$ of sample result

$\mathrm{U}_{\mathrm{R}}-\% \mathrm{RSD}$ greater than $15 \%$

$\mathrm{U}_{\mathrm{G}}-$ glass std result greater than $15 \%$ from expected result 
WSRC-TR-2005-00525, REV. 0

Table 3-9. Composition of the $3^{\text {rd }}$ Aqua Regia Dissolution of Solids from Tank 51 Sample HTF-076-81 (continued)

\begin{tabular}{|c|c|c|c|c|c|c|c|}
\hline Analyte (Method) & $\begin{array}{c}\text { 1st } \\
\text { Replicate } \\
(\mathrm{mg} / \mathrm{Kg})\end{array}$ & $\begin{array}{c}\text { 2nd } \\
\text { Replicate } \\
(\mathrm{mg} / \mathrm{Kg})\end{array}$ & $\begin{array}{c}\text { 3rd } \\
\text { Replicate } \\
(\mathrm{mg} / \mathrm{Kg})\end{array}$ & $\begin{array}{l}\text { Average } \\
\text { (mg/Kg) }\end{array}$ & $\%$ RSD & $\begin{array}{c}\text { Blank } \\
(\mathrm{mg} / \mathrm{Kg})\end{array}$ & $\begin{array}{c}\text { Data } \\
\text { Quality } \\
\text { Flags } \\
\end{array}$ \\
\hline Th (IM) & $3.19 \mathrm{E}+02$ & $3.29 \mathrm{E}+02$ & $3.23 \mathrm{E}+02$ & $3.24 \mathrm{E}+02$ & $1.7 \%$ & $<2.0 \mathrm{E}+00$ & - \\
\hline $\mathrm{Ru}$ (IM) & $<9.9 \mathrm{E}+01$ & $<9.3 \mathrm{E}+01$ & $<9.2 \mathrm{E}+01$ & $<9.5 \mathrm{E}+01$ & - & $<1.0 \mathrm{E}+02$ & - \\
\hline Ag (IM) & $<4.4 \mathrm{E}+01$ & $<4.1 \mathrm{E}+01$ & $<4.1 \mathrm{E}+01$ & $<4.2 \mathrm{E}+01$ & - & $<4.6 \mathrm{E}+01$ & - \\
\hline $\mathrm{Rh}$ (IM) & $4.22 \mathrm{E}+01$ & $4.84 \mathrm{E}+01$ & $4.70 \mathrm{E}+01$ & $4.59 \mathrm{E}+01$ & $7.2 \%$ & $<1.0 \mathrm{E}+00$ & - \\
\hline $\mathrm{Pd}$ (IM) & $<1.4 \mathrm{E}+00$ & $7.41 \mathrm{E}+00$ & $<1.3 \mathrm{E}+00$ & $7.41 \mathrm{E}+00 *$ & - & $<1.5 \mathrm{E}+00$ & $\mathrm{U}_{\mathrm{E}}$ \\
\hline $\mathrm{Tc}^{99}$ (IM) & $<2.0 \mathrm{E}+01$ & $<1.9 \mathrm{E}+01$ & $<1.9 \mathrm{E}+01$ & $<1.9 \mathrm{E}+01$ & - & $<2.1 \mathrm{E}+01$ & - \\
\hline $\mathrm{Np}^{237}$ (IM) & $6.47 \mathrm{E}+00$ & $7.63 \mathrm{E}+00$ & $6.48 \mathrm{E}+00$ & $6.86 \mathrm{E}+00$ & $9.7 \%$ & $<1.0 \mathrm{E}+00$ & $\mathrm{U}_{\mathrm{E}}$ \\
\hline $\mathrm{Pu}^{239}$ (IM) & $4.17 \mathrm{E}+01$ & $4.65 \mathrm{E}+01$ & $4.04 \mathrm{E}+01$ & $4.29 \mathrm{E}+01$ & $7.4 \%$ & $<1.0 \mathrm{E}+00$ & - \\
\hline $\mathrm{Pu}^{240}(\mathrm{IM})$ & $7.62 \mathrm{E}+00$ & $6.13 \mathrm{E}+00$ & $6.26 \mathrm{E}+00$ & $6.67 \mathrm{E}+00$ & $12 \%$ & $<1.0 \mathrm{E}+00$ & $\mathrm{U}_{\mathrm{E}}$ \\
\hline $\mathrm{Am}^{241} / \mathrm{Pu}^{241}$ (IM) & $<9.5 \mathrm{E}-01$ & $<8.9 \mathrm{E}-01$ & $<8.8 \mathrm{E}-01$ & $<9.1 \mathrm{E}-01$ & - & $<1.0 \mathrm{E}+00$ & - \\
\hline $\mathrm{Pu}^{242}(\mathrm{IM})$ & $<1.4 \mathrm{E}+00$ & $<1.3 \mathrm{E}+00$ & $<1.3 \mathrm{E}+00$ & $<1.4 \mathrm{E}+00$ & - & $<1.5 \mathrm{E}+00$ & - \\
\hline $\mathrm{Am}^{243}(\mathrm{IM})$ & $<9.5 \mathrm{E}-01$ & $<8.9 \mathrm{E}-01$ & $<8.8 \mathrm{E}-01$ & $<9.1 \mathrm{E}-01$ & - & $<1.0 \mathrm{E}+00$ & - \\
\hline $\mathrm{Cm}^{244}$ (IM) & $<9.5 \mathrm{E}-01$ & $<8.9 \mathrm{E}-01$ & $<8.8 \mathrm{E}-01$ & $<9.1 \mathrm{E}-01$ & - & $<1.0 \mathrm{E}+00$ & - \\
\hline $\mathrm{U}^{233}(\mathrm{IM})$ & $<9.5 \mathrm{E}-01$ & $<8.9 \mathrm{E}-01$ & $<8.8 \mathrm{E}-01$ & $<9.1 \mathrm{E}-01$ & - & $<1.0 \mathrm{E}+00$ & - \\
\hline $\mathrm{U}^{234}$ (IM) & $<9.5 \mathrm{E}-01$ & $4.56 \mathrm{E}+00$ & $<8.8 \mathrm{E}-01$ & $4.56 \mathrm{E}+00^{*}$ & - & $<1.0 \mathrm{E}+00$ & $\mathrm{U}_{\mathrm{E}}$ \\
\hline $\mathrm{U}^{235}(\mathrm{IM})$ & $6.50 \mathrm{E}+01$ & $6.73 \mathrm{E}+01$ & $6.43 \mathrm{E}+01$ & $6.55 \mathrm{E}+01$ & $2.4 \%$ & $<1.0 \mathrm{E}+00$ & - \\
\hline $\mathrm{U}^{236}$ (IM) & $6.33 \mathrm{E}+00$ & $5.90 \mathrm{E}+00$ & $6.01 \mathrm{E}+00$ & $6.08 \mathrm{E}+00$ & $3.6 \%$ & $<1.0 \mathrm{E}+00$ & $\mathrm{U}_{\mathrm{E}}$ \\
\hline $\mathrm{U}^{238}$ (IM) & $7.94 \mathrm{E}+03$ & $8.22 \mathrm{E}+03$ & $8.01 \mathrm{E}+03$ & $8.06 \mathrm{E}+03$ & $1.8 \%$ & $<1.0 \mathrm{E}+00$ & - \\
\hline U-total (sum) & $8.01 \mathrm{E}+03$ & $8.29 \mathrm{E}+03$ & $8.08 \mathrm{E}+03$ & $8.13 \mathrm{E}+03$ & $1.8 \%$ & - & - \\
\hline
\end{tabular}

* Single Value

Data Quality Flags:
$\mathrm{U}_{\mathrm{E}}$ - result is less than $10 \mathrm{X}$ the detection limit

$\mathrm{U}_{\mathrm{B}}$ - blank result greater than $5 \%$ of sample result
$\mathrm{U}_{\mathrm{R}}-\% \mathrm{RSD}$ greater than $15 \%$

$\mathrm{U}_{\mathrm{G}}-$ glass std result greater than $15 \%$ from expected result 
WSRC-TR-2005-00525, REV. 0

Table 3-10. Composition of the $3^{\text {rd }}$ Sodium Peroxide Fusion Dissolution of Solids from Tank 51 Sample HTF-076-81

\begin{tabular}{|c|c|c|c|c|c|c|c|c|c|}
\hline Analyte (Method) & $\begin{array}{c}\text { 1st } \\
\text { Replicate } \\
(\mathrm{mg} / \mathrm{Kg})\end{array}$ & $\begin{array}{c}\text { 2nd } \\
\text { Replicate } \\
(\mathrm{mg} / \mathrm{Kg})\end{array}$ & $\begin{array}{c}\text { 3rd } \\
\text { Replicate } \\
(\mathrm{mg} / \mathrm{Kg})\end{array}$ & $\begin{array}{l}\text { Average } \\
\text { (mg/Kg) }\end{array}$ & $\%$ RSD & $\begin{array}{c}\text { Blank } \\
(\mathrm{mg} / \mathrm{Kg})\end{array}$ & $\begin{array}{c}\text { Analyzed } \\
\text { Glass Std } \\
(\mathrm{mg} / \mathrm{Kg})\end{array}$ & $\begin{array}{c}\text { Glass Std } \\
\text { Composition } \\
(\mathrm{mg} / \mathrm{Kg})\end{array}$ & $\begin{array}{c}\text { Data } \\
\text { Quality } \\
\text { Flags } \\
\end{array}$ \\
\hline $\mathrm{Ag}$ (IE) & $<6.3 \mathrm{E}+02$ & $<6.5 \mathrm{E}+02$ & $6.21 \mathrm{E}+02$ & $6.21 \mathrm{E}+02 *$ & - & $7.44 \mathrm{E}+02$ & $<6.9 \mathrm{E}+02$ & - & $\mathrm{U}_{\mathrm{B}} \mathrm{U}_{\mathrm{E}}$ \\
\hline Al (IE) & $7.56 \mathrm{E}+04$ & $7.20 \mathrm{E}+04$ & $7.71 \mathrm{E}+04$ & $7.49 \mathrm{E}+04$ & $3.5 \%$ & $<4.2 \mathrm{E}+02$ & $2.41 \mathrm{E}+04$ & $2.50 \mathrm{E}+04$ & - \\
\hline B (IE) & $<4.8 \mathrm{E}+02$ & $<5.0 \mathrm{E}+02$ & $<4.7 \mathrm{E}+02$ & $<4.8 \mathrm{E}+02$ & - & $<5.3 \mathrm{E}+02$ & $2.60 \mathrm{E}+04$ & $2.69 \mathrm{E}+04$ & - \\
\hline $\mathrm{Ba}$ (IE) & $3.37 \mathrm{E}+02$ & $3.21 \mathrm{E}+02$ & $3.78 \mathrm{E}+02$ & $3.45 \mathrm{E}+02$ & $8.4 \%$ & $<1.1 \mathrm{E}+02$ & $8.85 \mathrm{E}+02$ & $7.90 \mathrm{E}+02$ & $\mathrm{U}_{\mathrm{E}}$ \\
\hline $\mathrm{Ca}$ (IE) & $5.84 \mathrm{E}+03$ & $6.38 \mathrm{E}+03$ & $6.27 \mathrm{E}+03$ & $6.16 \mathrm{E}+03$ & $4.6 \%$ & $2.09 \mathrm{E}+03$ & $1.26 \mathrm{E}+04$ & $1.02 \mathrm{E}+04$ & $\mathrm{U}_{\mathrm{G}} \mathrm{U}_{\mathrm{B}}$ \\
\hline $\mathrm{Cd}$ (IE) & $<1.4 \mathrm{E}+02$ & $<1.4 \mathrm{E}+02$ & $<1.3 \mathrm{E}+02$ & $<1.4 \mathrm{E}+02$ & - & $<1.5 \mathrm{E}+02$ & $<1.5 \mathrm{E}+02$ & - & - \\
\hline $\mathrm{Ce}$ (IE) & $8.58 \mathrm{E}+03$ & $7.76 \mathrm{E}+03$ & $9.49 \mathrm{E}+03$ & $8.61 \mathrm{E}+03$ & $10 \%$ & $6.97 \mathrm{E}+03$ & $7.02 \mathrm{E}+03$ & - & $\mathrm{U}_{\mathrm{B}} \mathrm{U}_{\mathrm{E}}$ \\
\hline $\mathrm{Cr}$ (IE) & $<3.2 \mathrm{E}+02$ & $<3.3 \mathrm{E}+02$ & $3.58 \mathrm{E}+02$ & $3.58 \mathrm{E}+02 *$ & - & $<3.6 \mathrm{E}+02$ & $6.18 \mathrm{E}+02$ & $6.40 \mathrm{E}+02$ & $\mathrm{U}_{\mathrm{E}}$ \\
\hline $\mathrm{Cu}$ (IE) & $1.25 \mathrm{E}+02$ & $1.18 \mathrm{E}+02$ & $3.14 \mathrm{E}+02$ & $1.85 \mathrm{E}+02$ & $60 \%$ & $<1.1 \mathrm{E}+02$ & $3.01 \mathrm{E}+03$ & $3.00 \mathrm{E}+01$ & $\mathrm{U}_{\mathrm{G}} \mathrm{U}_{\mathrm{R}} \mathrm{U}_{\mathrm{E}}$ \\
\hline $\mathrm{Fe}$ (IE) & $3.73 \mathrm{E}+04$ & $3.64 \mathrm{E}+04$ & $3.85 \mathrm{E}+04$ & $3.74 \mathrm{E}+04$ & $2.8 \%$ & $1.55 \mathrm{E}+02$ & $1.01 \mathrm{E}+05$ & $9.79 \mathrm{E}+04$ & - \\
\hline K (IE) & $1.92 \mathrm{E}+04$ & $1.91 \mathrm{E}+04$ & $2.94 \mathrm{E}+04$ & $2.26 \mathrm{E}+04$ & $26 \%$ & $2.05 \mathrm{E}+04$ & $3.73 \mathrm{E}+04$ & $2.26 \mathrm{E}+04$ & $\mathrm{U}_{\mathrm{G}} \mathrm{U}_{\mathrm{B}} \mathrm{U}_{\mathrm{R}} \mathrm{U}_{\mathrm{E}}$ \\
\hline La (IE) & $1.31 \mathrm{E}+03$ & $1.10 \mathrm{E}+03$ & $1.39 \mathrm{E}+03$ & $1.27 \mathrm{E}+03$ & $12 \%$ & $8.77 \mathrm{E}+02$ & $9.48 \mathrm{E}+02$ & - & $\mathrm{U}_{\mathrm{B}} \mathrm{U}_{\mathrm{E}}$ \\
\hline Li (IE) & $1.09 \mathrm{E}+03$ & $1.16 \mathrm{E}+03$ & $1.33 \mathrm{E}+03$ & $1.19 \mathrm{E}+03$ & $10 \%$ & $9.73 \mathrm{E}+02$ & $1.66 \mathrm{E}+04$ & $1.49 \mathrm{E}+04$ & $\mathrm{U}_{\mathrm{B}} \mathrm{U}_{\mathrm{E}}$ \\
\hline $\mathrm{Mg}$ (IE) & $1.35 \mathrm{E}+03$ & $1.32 \mathrm{E}+03$ & $1.41 \mathrm{E}+03$ & $1.36 \mathrm{E}+03$ & $3.4 \%$ & $<4.0 \mathrm{E}+01$ & $5.08 \mathrm{E}+03$ & $5.20 \mathrm{E}+03$ & - \\
\hline $\mathrm{Mn}$ (IE) & $8.97 \mathrm{E}+03$ & $8.74 \mathrm{E}+03$ & $9.31 \mathrm{E}+03$ & $9.01 \mathrm{E}+03$ & $3.2 \%$ & $<2.0 \mathrm{E}+01$ & $1.49 \mathrm{E}+04$ & $1.46 \mathrm{E}+04$ & - \\
\hline Mo (IE) & $<1.2 \mathrm{E}+03$ & $<1.2 \mathrm{E}+03$ & $1.36 \mathrm{E}+03$ & $1.36 \mathrm{E}+03 *$ & - & $<1.3 \mathrm{E}+03$ & $1.48 \mathrm{E}+03$ & - & $\mathrm{U}_{\mathrm{E}}$ \\
\hline
\end{tabular}

* Single Value Divide $\mathrm{mg} / \mathrm{Kg}$ values by $1 \mathrm{E}+04$ to convert to $\mathrm{wt} \%$ basis

Data Quality Flags: $\quad \mathrm{U}_{\mathrm{E}}$ - result is less than $10 \mathrm{X}$ the detection limit $\quad \mathrm{U}_{\mathrm{R}}-$ \%RSD greater than $15 \%$

$\begin{array}{ll}U_{B}-\text { blank result greater than } 5 \% \text { of sample result } & U_{G}-\text { glass std result greater than } 15 \% \text { from expected result }\end{array}$ 
WSRC-TR-2005-00525, REV. 0

Table 3-10. Composition of the $3^{\text {rd }}$ Sodium Peroxide Fusion Dissolution of Solids from Tank 51 Sample HTF-076-81 (continued)

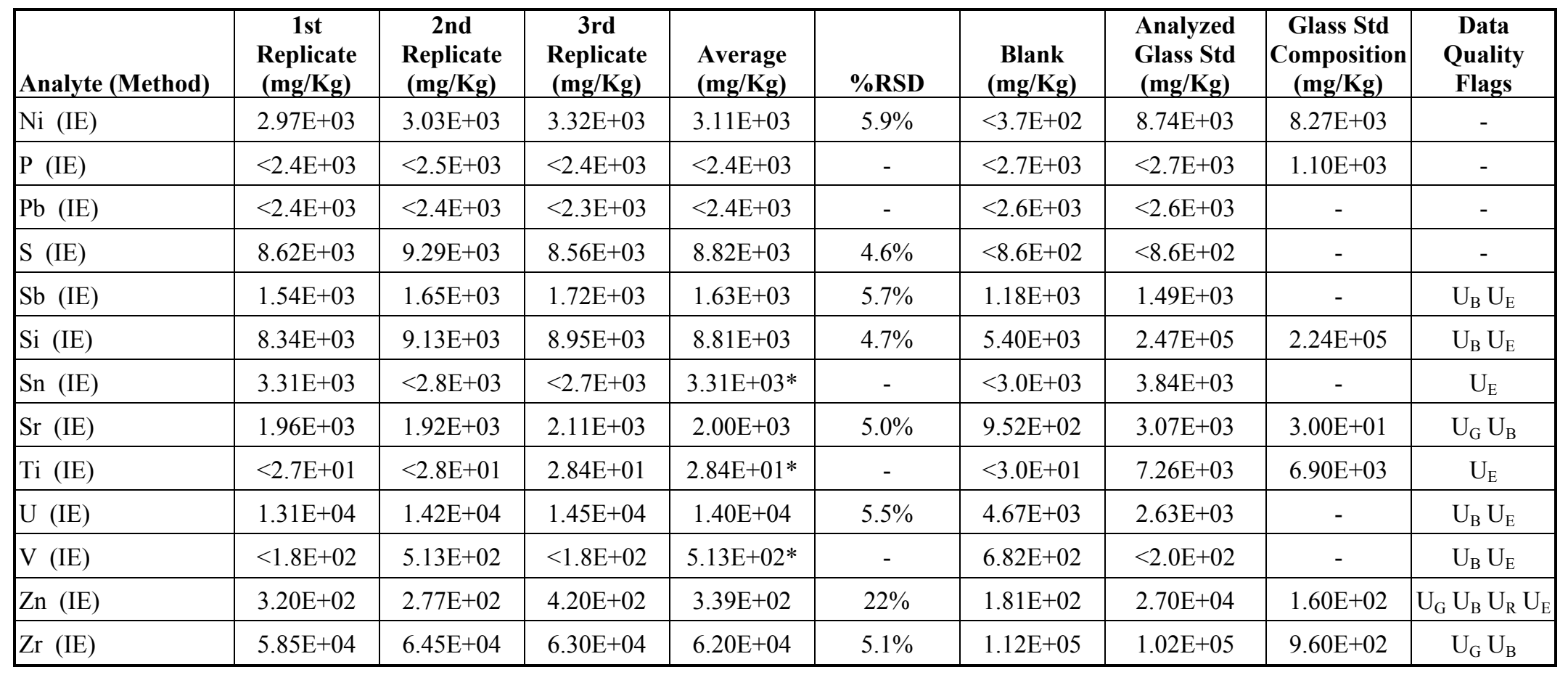

* Single Value

Data Quality Flags:
Divide $\mathrm{mg} / \mathrm{Kg}$ values by $1 \mathrm{E}+04$ to convert to $\mathrm{wt} \%$ basis

$\mathrm{U}_{\mathrm{E}}$ - result is less than $10 \mathrm{X}$ the detection limit

$\mathrm{U}_{\mathrm{B}}$ - blank result greater than $5 \%$ of sample result
$\mathrm{U}_{\mathrm{R}}-\% \mathrm{RSD}$ greater than $15 \%$

$\mathrm{U}_{\mathrm{G}}-$ glass std result greater than $15 \%$ from expected result 
WSRC-TR-2005-00525, REV. 0

Table 3-10. Composition of the $3^{\text {rd }}$ Sodium Peroxide Fusion Dissolution of Solids from Tank 51 Sample HTF-076-81 (continued)

\begin{tabular}{|c|c|c|c|c|c|c|c|}
\hline Analyte (Method) & $\begin{array}{c}\text { 1st } \\
\text { Replicate } \\
(\mathrm{mg} / \mathrm{Kg})\end{array}$ & $\begin{array}{c}\text { 2nd } \\
\text { Replicate } \\
(\mathrm{mg} / \mathrm{Kg})\end{array}$ & $\begin{array}{c}\text { 3rd } \\
\text { Replicate } \\
(\mathrm{mg} / \mathrm{Kg})\end{array}$ & $\begin{array}{l}\text { Average } \\
\text { (mg/Kg) }\end{array}$ & $\%$ RSD & $\begin{array}{c}\text { Blank } \\
(\mathrm{mg} / \mathrm{Kg})\end{array}$ & $\begin{array}{c}\text { Data } \\
\text { Quality } \\
\text { Flags }\end{array}$ \\
\hline Th (IM) & $3.40 \mathrm{E}+02$ & $3.33 \mathrm{E}+02$ & $3.42 \mathrm{E}+02$ & $3.39 \mathrm{E}+02$ & $1.3 \%$ & $<2.8 \mathrm{E}+00$ & - \\
\hline $\mathrm{Ru}$ (IM) & $4.11 \mathrm{E}+02$ & $4.14 \mathrm{E}+02$ & $4.38 \mathrm{E}+02$ & $4.21 \mathrm{E}+02$ & $3.5 \%$ & $<4.2 \mathrm{E}+01$ & - \\
\hline Ag (IM) & $2.96 \mathrm{E}+01$ & $4.32 \mathrm{E}+01$ & $3.37 \mathrm{E}+01$ & $3.55 \mathrm{E}+01$ & $20 \%$ & $1.89 \mathrm{E}+01$ & $\mathrm{U}_{\mathrm{R}} \mathrm{U}_{\mathrm{B}}$ \\
\hline $\mathrm{Rh}$ (IM) & $4.57 \mathrm{E}+01$ & $4.76 \mathrm{E}+01$ & $5.05 \mathrm{E}+01$ & $4.79 \mathrm{E}+01$ & $5.1 \%$ & $<5.0 \mathrm{E}-01$ & - \\
\hline $\mathrm{Pd}$ (IM) & $7.94 \mathrm{E}+00$ & $7.39 \mathrm{E}+00$ & $8.32 \mathrm{E}+00$ & $7.39 \mathrm{E}+00$ & $6.3 \%$ & $<7.5 \mathrm{E}-01$ & - \\
\hline $\mathrm{Tc}^{99}$ (IM) & $1.10 \mathrm{E}+01$ & $1.11 \mathrm{E}+01$ & $1.13 \mathrm{E}+01$ & $1.12 \mathrm{E}+01$ & $1.1 \%$ & $<8.0 \mathrm{E}+00$ & - \\
\hline $\mathrm{Np}^{237}$ (IM) & $5.86 \mathrm{E}+00$ & $5.75 \mathrm{E}+00$ & $5.76 \mathrm{E}+00$ & $5.79 \mathrm{E}+00$ & $1.1 \%$ & $<5.0 \mathrm{E}-01$ & - \\
\hline $\mathrm{Pu}^{239}$ (IM) & $4.95 \mathrm{E}+01$ & $4.76 \mathrm{E}+01$ & $4.87 \mathrm{E}+01$ & $4.86 \mathrm{E}+01$ & $1.9 \%$ & $<5.0 \mathrm{E}-01$ & - \\
\hline $\mathrm{Pu}^{240}(\mathrm{IM})$ & $6.98 \mathrm{E}+00$ & $6.51 \mathrm{E}+00$ & $6.55 \mathrm{E}+00$ & $6.68 \mathrm{E}+00$ & $3.8 \%$ & $<5.0 \mathrm{E}-01$ & - \\
\hline $\mathrm{Am}^{241} / \mathrm{Pu}^{241}$ (IM) & $2.83 \mathrm{E}+00$ & $2.84 \mathrm{E}+00$ & $2.94 \mathrm{E}+00$ & $2.87 \mathrm{E}+00$ & $2.1 \%$ & $<5.0 \mathrm{E}-01$ & $\mathrm{U}_{\mathrm{E}}$ \\
\hline $\mathrm{Pu}^{242}(\mathrm{IM})$ & $5.35 \mathrm{E}-01$ & $5.83 \mathrm{E}-01$ & $5.86 \mathrm{E}-01$ & $5.68 \mathrm{E}-01$ & $5.0 \%$ & $<5.0 \mathrm{E}-01$ & $\mathrm{U}_{\mathrm{E}}$ \\
\hline $\mathrm{Am}^{243}$ (IM) & $8.43 \mathrm{E}-01$ & $7.86 \mathrm{E}-01$ & $7.70 \mathrm{E}-01$ & $7.99 \mathrm{E}-01$ & $4.8 \%$ & $<5.0 \mathrm{E}-01$ & - \\
\hline $\mathrm{Cm}^{244}$ (IM) & $<4.5 \mathrm{E}-01$ & $<4.7 \mathrm{E}-01$ & $<4.4 \mathrm{E}-01$ & $<4.6 \mathrm{E}-01$ & - & $<5.0 \mathrm{E}-01$ & - \\
\hline $\mathrm{U}^{233}$ (IM) & $8.58 \mathrm{E}-01$ & $8.11 \mathrm{E}-01$ & $8.14 \mathrm{E}-01$ & $8.28 \mathrm{E}-01$ & $3.2 \%$ & $<5.0 \mathrm{E}-01$ & $\mathrm{U}_{\mathrm{E}}$ \\
\hline $\mathrm{U}^{234}$ (IM) & $3.92 \mathrm{E}+00$ & $3.89 \mathrm{E}+00$ & $4.00 \mathrm{E}+00$ & $3.94 \mathrm{E}+00$ & $1.4 \%$ & $<5.0 \mathrm{E}-01$ & $\mathrm{U}_{\mathrm{E}}$ \\
\hline $\mathrm{U}^{235}$ (IM) & $6.93 \mathrm{E}+01$ & $6.64 \mathrm{E}+01$ & $6.77 \mathrm{E}+01$ & $6.78 \mathrm{E}+01$ & $2.1 \%$ & $<5.0 \mathrm{E}-01$ & - \\
\hline $\mathrm{U}^{236}$ (IM) & $5.45 \mathrm{E}+00$ & $5.45 \mathrm{E}+00$ & $6.05 \mathrm{E}+00$ & $5.65 \mathrm{E}+00$ & $6.1 \%$ & $<5.0 \mathrm{E}-01$ & - \\
\hline $\mathrm{U}^{238}$ (IM) & $8.66 \mathrm{E}+03$ & $8.19 \mathrm{E}+03$ & $8.45 \mathrm{E}+03$ & $8.44 \mathrm{E}+03$ & $2.8 \%$ & $<9.7 \mathrm{E}-01$ & - \\
\hline U-total (sum) & $8.74 \mathrm{E}+03$ & $8.27 \mathrm{E}+03$ & $8.53 \mathrm{E}+03$ & $8.51 \mathrm{E}+03$ & $2.8 \%$ & - & - \\
\hline
\end{tabular}

$\begin{array}{lll}\text { Data Quality Flags: } & \mathrm{U}_{\mathrm{E}}-\text { result is less than } 10 \mathrm{X} \text { the detection limit } & \mathrm{U}_{\mathrm{R}}-\% \text { RSD greater than } 15 \% \\ & \mathrm{U}_{\mathrm{B}}-\text { blank result greater than } 5 \% \text { of sample result } & \mathrm{U}_{\mathrm{G}}-\text { glass std result greater than } 15 \% \text { from expected result }\end{array}$ 
WSRC-TR-2005-00525, REV. 0

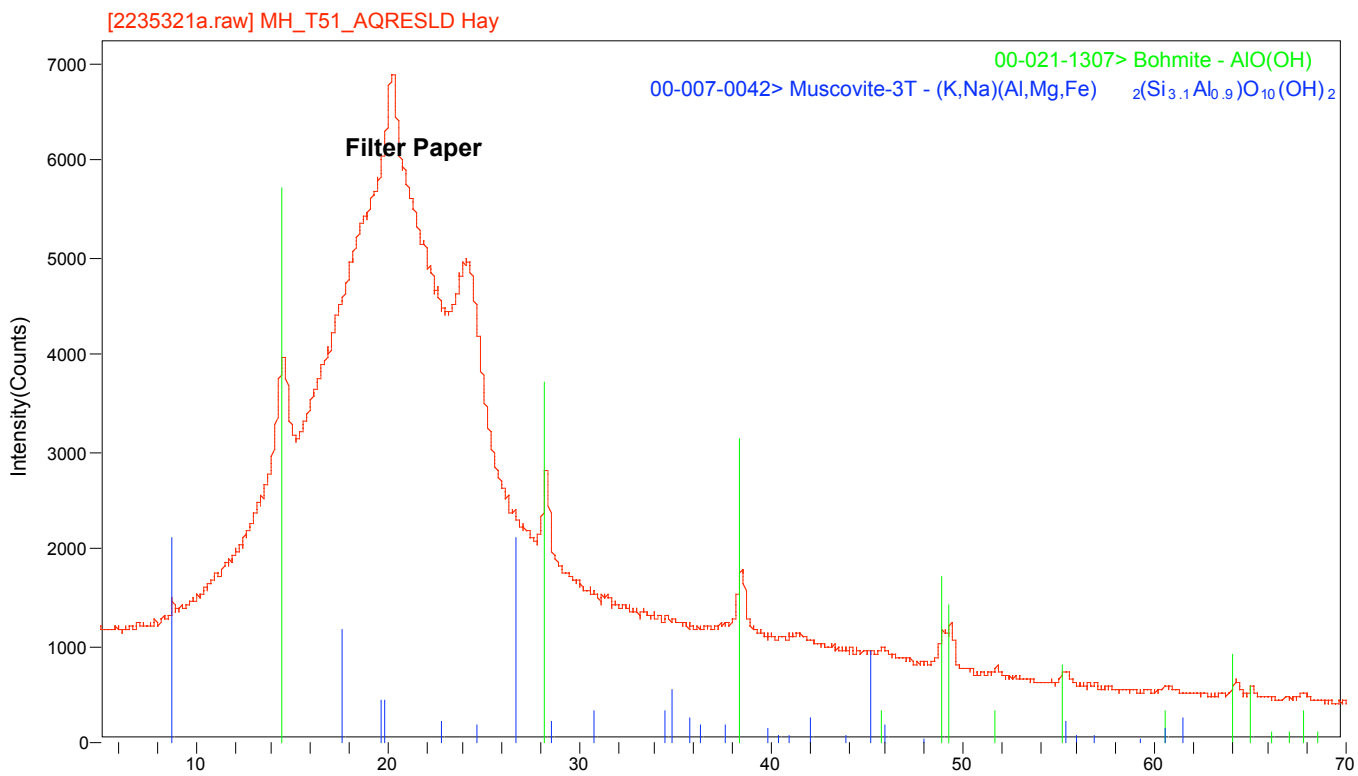

Figure 3-1. XRD of Undissolved Solids Filtered from Aqua-Regia Dissolutions 
WSRC-TR-2005-00525, REV. 0

\subsection{SLUDGE SETTLING TESTS}

\subsection{INITIAL SETTLING TEST OF TANK 51H SLUDGE}

Figure 4-1 shows the initial settling data collected after compositing the Tank $51 \mathrm{H}$ slurry samples in a calibrated $1 \mathrm{~L}$ poly bottle. The data have been plotted as percent of the initial sludge slurry volume in the vessel. The figure also shows a comparison of Tank $51 \mathrm{H}$ sludge settling rate to the settling rate of a Sludge Batch 3 (SB3) sample conducted in $100 \mathrm{~mL}$ glass graduated cylinders. The sludge from SB3 settled much faster than Tank 51H sludge. Comparing the settling rate of Tank $51 \mathrm{H}$ sludge in the $1 \mathrm{~L}$ poly bottle versus the much narrower $100 \mathrm{~mL}$ glass graduated cylinder indicates the slower settling rate in the narrow graduated cylinder may be due to either the narrowness of the vessel or the tendency for sludge to stick to glass more than plastic. Both the rate of settling and the ultimate settled height appear to be negatively affected. The initial slurry height to diameter (L/D) for the $1 \mathrm{~L}$ poly bottle settling test was $\sim 1$ while the $100 \mathrm{~mL}$ glass graduated cylinder settling test had a $\mathrm{L} / \mathrm{D}$ of $\sim 7$. For comparison, the L/D in Tank $51 \mathrm{H}$ when the sample was obtained from the tank was approximately 0.24 based on a slurry height of 247" and tank diameter of 85 feet. The much larger L/D for the $100 \mathrm{~mL}$ glass graduated cylinder settling test might also have impacted the test results. The tall, narrow $100 \mathrm{~mL}$ graduated cylinder was chosen for the settling test to allow more accurate determination of the settled sludge height using a relatively small amount of sample.

Past experience would predict that the high aluminum sludge in Tank $51 \mathrm{H}$ should settle slower than the high iron sludge in SB3. F-Area high iron sludge tends to settle faster and to a higher solids content than $\mathrm{H}$-area high aluminum sludge. ${ }^{10}$ However, the magnitude of the difference observed in the current case might be related to sludge retrieval operations from Tank $11 \mathrm{H}$ where large amounts of water and high shear were needed to remove and transfer the sludge to Tank $51 \mathrm{H}$. Past sludge washing experiments found that when the slurrying solution becomes too depleted in salt, the sludge breaks up into fine particles that settle very slowly. ${ }^{11}$

\subsection{BLENDING TANK 51H SLUDGE WITH SB3 SLUDGE}

Since the SB3 sludge settled very quickly and to a high solids concentration, and because high iron sludge from Tanks $5 \mathrm{~F}$ and $6 \mathrm{~F}$ will be added to Tank $51 \mathrm{H}$ as part of SB4, a blend of Tank $51 \mathrm{H}$ sludge and SB3 sludge was prepared to determine the effect on the sludge settling rate. The SB3 sludge was added to a quantity of Tank $51 \mathrm{H}$ sludge to provide similar proportions of $\mathrm{H}$-Area and $\mathrm{F}$-Area sludge projected after the addition of Tank $5 \mathrm{~F}$ and $6 \mathrm{~F}$ sludge to Tank $51 \mathrm{H}$. A projected composition of Tank $51 \mathrm{H}$ after the addition of Tanks $5 \mathrm{~F}$ and $6 \mathrm{~F}$ was provided by J. Gillam. ${ }^{12}$ The SB3 sludge used for the blend was different in terms of weight percent solids and supernate composition compared with the projected composition of the material from Tanks $5 \mathrm{~F}$ and $6 \mathrm{~F}$. Therefore, it was not possible to completely match the projected composition for Tank $51 \mathrm{H}$ after the addition of the $5 \mathrm{~F} / 6 \mathrm{~F}$ sludge. To compare settling rates, the weight percent insoluble solids of slurry and the supernate composition of the slurry were of primary importance. Therefore, a blend of Tank 
51H and the SB3 sludge was targeted to match the weight percent insoluble solids, a free hydroxide concentration of approximately $1.0 \mathrm{M}$, and a sodium concentration in the supernate of approximately $3.0 \mathrm{M}$. Table $4-1$ shows the projected targets for the Tank $51 \mathrm{H}$ sample after the addition of Tanks 5F and $6 \mathrm{~F}$ and the calculated values for the blend of Tank $51 \mathrm{H}$ and SB3 sludge. The Tank 51H/SB3 blend used in the settling tests has a higher ratio of SB3 (high iron sludge) to Tank $51 \mathrm{H}$ sludge solids (high aluminum sludge) than the projection for Tank $51 \mathrm{H}$ after the addition of sludge from Tanks $5 \mathrm{~F}$ and $6 \mathrm{~F}$. Therefore, any effect on the settling rate should be more pronounced in the experimental Tank 51H/SB3 blend. The settling tests were conducted in $500 \mathrm{~mL}$ glass graduated cylinders to attempt to decrease wall effects. Figure 4-2 shows the settling rate of the Tank $51 \mathrm{H} / \mathrm{SB} 3$ blend in comparison to Tank $51 \mathrm{H}$ alone. The settling data for Tank $51 \mathrm{H}$ in a $1 \mathrm{~L}$ poly bottle from Figure $4-1$ has also been plotted for reference.

From the graph, adding high iron sludge (SB3) to the Tank 51H sludge appears to have little effect on the settling rate and the final settled sludge volume. In comparing the Tank $51 \mathrm{H}$ sludge in the $500 \mathrm{~mL}$ glass graduated cylinder versus the same sludge in a $1 \mathrm{~L}$ poly bottle, the data seems to indicate that the glass of the graduated cylinder may still be negatively affecting the sludge settling rate. The $\mathrm{L} / \mathrm{D}$ ratio for the $500 \mathrm{~mL}$ graduated cylinder settling test of $\sim 2.5$ was much closer to the $\mathrm{L} / \mathrm{D}$ of the $1 \mathrm{~L}$ poly bottle.

\subsection{HEAT TREATMENT OF TANK 51H AND TANK 51/SB3 BLEND SLUDGE}

The wastewater treatment industry uses heat treatment concentrations on slow settling slurries as a method to increase the agglomeration of fine particles and, thereby, increase the settling rate. An experiment was conducted to try and improve the settling rate of Tank $51 \mathrm{H}$ high aluminum sludge by adding caustic and heating the sludge. The addition of heat and caustic could increase the solubility of aluminum in the slurry and possibly lead to conditions that encourage crystal growth. After discussions with the customer about the practical limits for temperature and caustic additions to the tank, a temperature of $60-65^{\circ} \mathrm{C}$ and $1 \mathrm{M}$ free hydroxide was established as within current operating limits for the tank. A portion of the Tank $51 \mathrm{H}$ sludge was adjusted to a $1 \mathrm{M}$ free hydroxide concentration by adding a small amount of $50 \mathrm{wt} \% \mathrm{NaOH}$. The sludge was then heated to $60-65^{\circ} \mathrm{C}$ for four days in a sealed pressure vessel. A portion of the Tank $51 \mathrm{H} / \mathrm{SB} 3$ blend sludge, already at $1 \mathrm{M}$ free hydroxide, was also heated to $60-65^{\circ}$ for four days. A settling test was conducted to compare the settling rates of heat-treated and untreated sludge. The settling tests were conducted in plastic 250 $\mathrm{mL}$ graduated cylinders to eliminate the negative effects found with glass vessels on the settling rate. The $\mathrm{L} / \mathrm{D}$ ratio for the $250 \mathrm{~mL}$ plastic graduated cylinder settling test was $\sim 1$.

Figure 4-3 shows the results of the settling test between heat-treated and untreated Tank $51 \mathrm{H}$ and Tank 51/SB3 blend sludge. A similar volume of untreated sludge was allowed to settle in identical vessels so the results would be directly comparable. The graph seems to indicate that heat treatment might have produced a small positive effect on the settling rate for both the Tank 51H sludge and the Tank 51H/SB3 blend. However, evaluating all of the Tank 51H settling data together in Figure 4-4, indicates that the effect from heat treatment might not be statistically significant. Comparing the settling curves of Tank $51 \mathrm{H}$ sludge in the $1 \mathrm{~L}$ poly 
bottle and in the $250 \mathrm{~mL}$ plastic graduated cylinder, which both had the same L/D ratio, show nearly as much difference.

\subsection{CONCLUSIONS FROM THE SETTLING TESTS}

Settling tests conducted with the Tank $51 \mathrm{H}$ sludge showed a much slower settling rate than with the sludge in Sludge Batch 3 (SB3). A mixture of Tank 51H and sludge from SB3 was prepared to mimic the projected final composition of Sludge Batch 4 (SB4). The mixture showed minimal improvement in the settling rate versus Tank $51 \mathrm{H}$ sludge alone. An attempt to increase the settling rate of Tank $51 \mathrm{H}$ sludge by adjusting the sludge to a $1.0 \mathrm{M}$ free hydroxide concentration and heating to $60-65^{\circ} \mathrm{C}$ for four days also showed no significant improvement in the settling rate.

Higher temperatures, higher caustic concentrations, and longer heating times may be required to significantly improve the settling rate of the Tank $51 \mathrm{H}$ sludge. However, limitations in Tank Farm due to safety and corrosion concerns may preclude reaching a temperature and/or caustic concentration that could increase the sludge settling rate. Modeling could be used to investigate the effect of concentration, $\mathrm{pH}$, and temperature on the solid phases present in the sludge solids and develop regions for further experimentation. Limited flocculent addition testing has been performed with simulants to try and improve the settling rate and has been documented in a separate memorandum. ${ }^{13}$ Testing of additional additives with sludge simulants is being pursued and any found to be successful will be tested with the Tank $51 \mathrm{H}$ sludge sample. 
WSRC-TR-2005-00525, REV. 0

Table 4-1. Target Composition for Tank 51H after Addition of Tank 5F and 6F Compared with the Calculated Composition of the Tank 51H/SB3 Blend.

\begin{tabular}{|l|c|c|}
\hline & $\begin{array}{c}\text { Projected Tank 51H/5F/6F } \\
\text { Composition }\end{array}$ & $\begin{array}{c}\text { Calc. Tank 51H/SB3 Blend } \\
\text { Composition }\end{array}$ \\
\hline $\mathrm{Wt}^{\circ}$ Insoluble Solids & 10.16 & 10.23 \\
\hline $\mathrm{OH}_{\text {free }}^{-}$ & 1.00 & 1.01 \\
\hline $\mathrm{Na}^{+}$ & 3.27 & 2.68 \\
\hline $\begin{array}{l}\text { Estimated Weight Ratio of } \\
\begin{array}{l}\text { High Al Sludge to High } \\
\text { Fe Sludge }\end{array}\end{array}$ & 2.2 & 1.0 \\
\hline
\end{tabular}


Initial Settling of Tank $51 \mathrm{H}$ Sludge in $1 \mathrm{~L}$ Poly Bottle

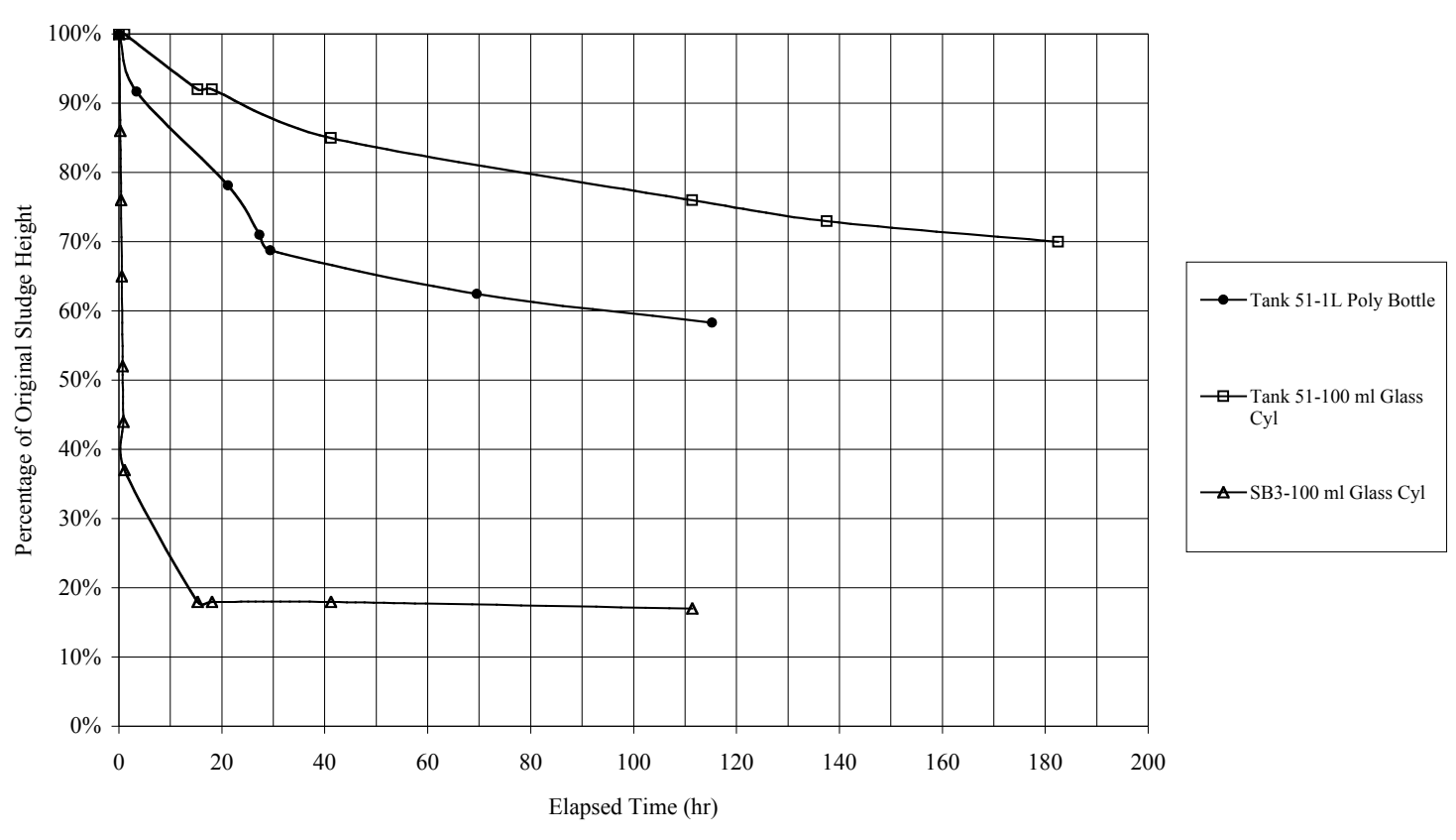

Figure 4-1. Initial Tank 51H Sludge Settling 


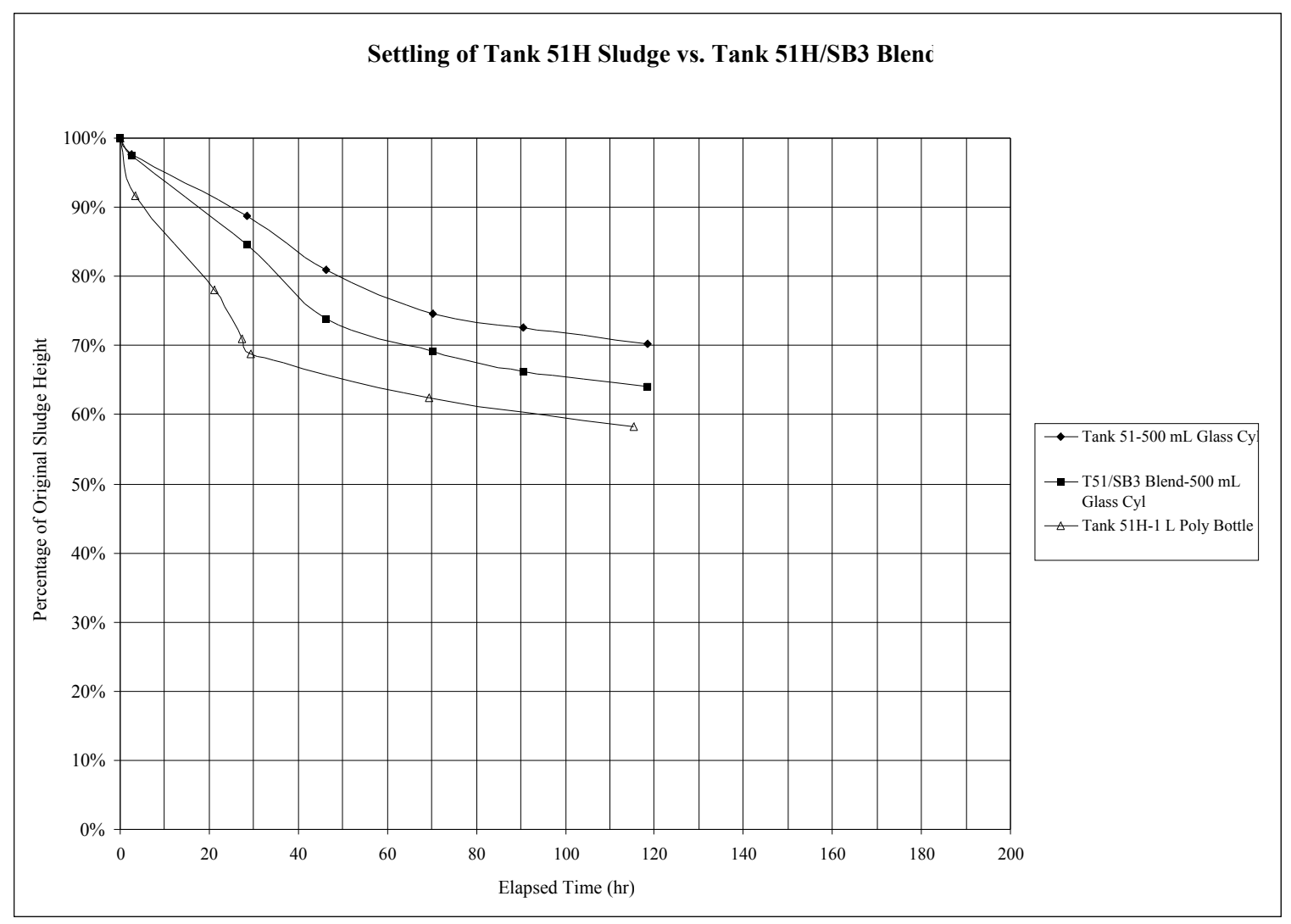

Figure 4-2. Tank 51H Sludge Settling versus a Blend of Tank 51H and SB3 Sludge 


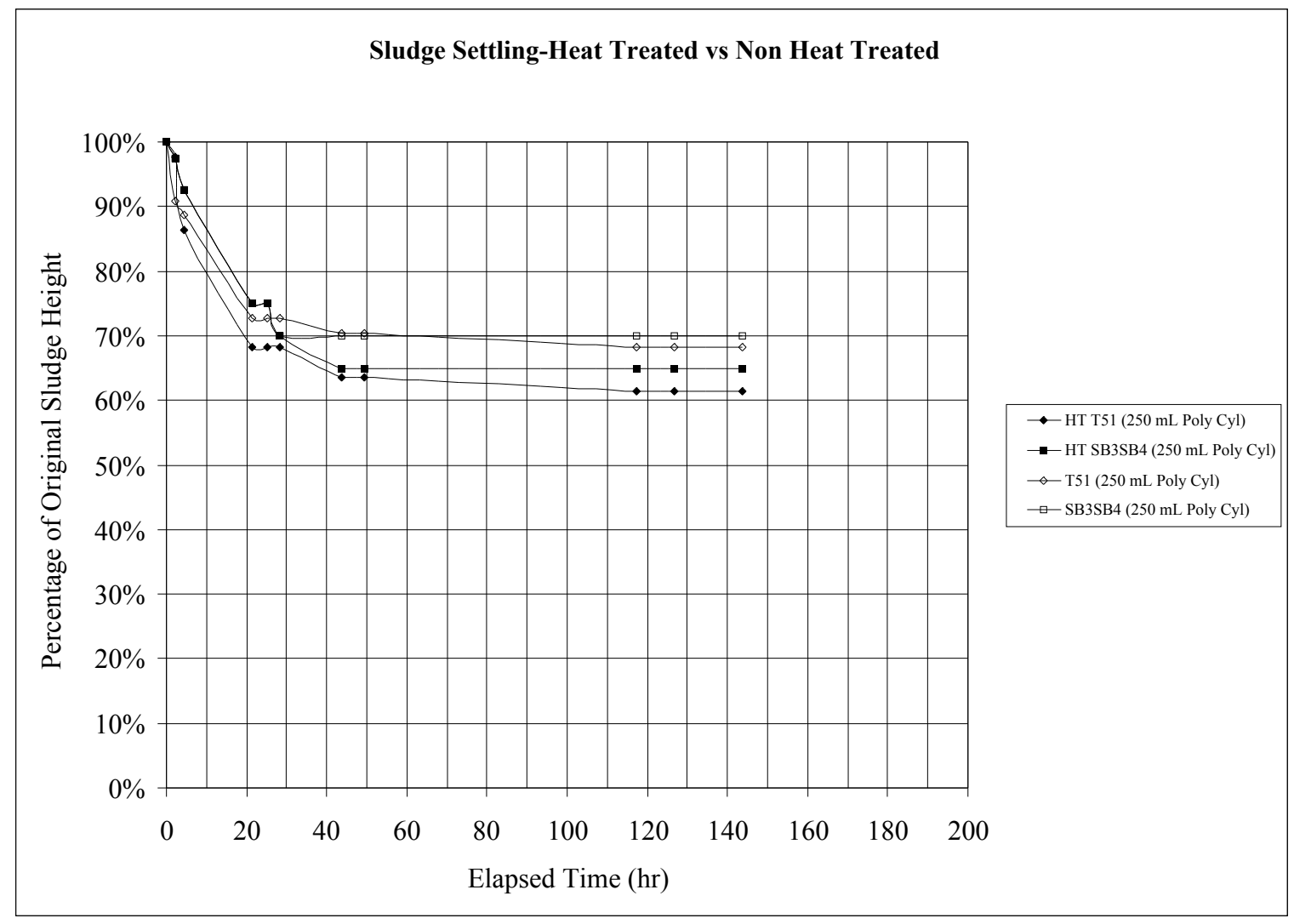

Figure 4-3. Heat Treated versus Non-Heat Treated Sludge Settling 


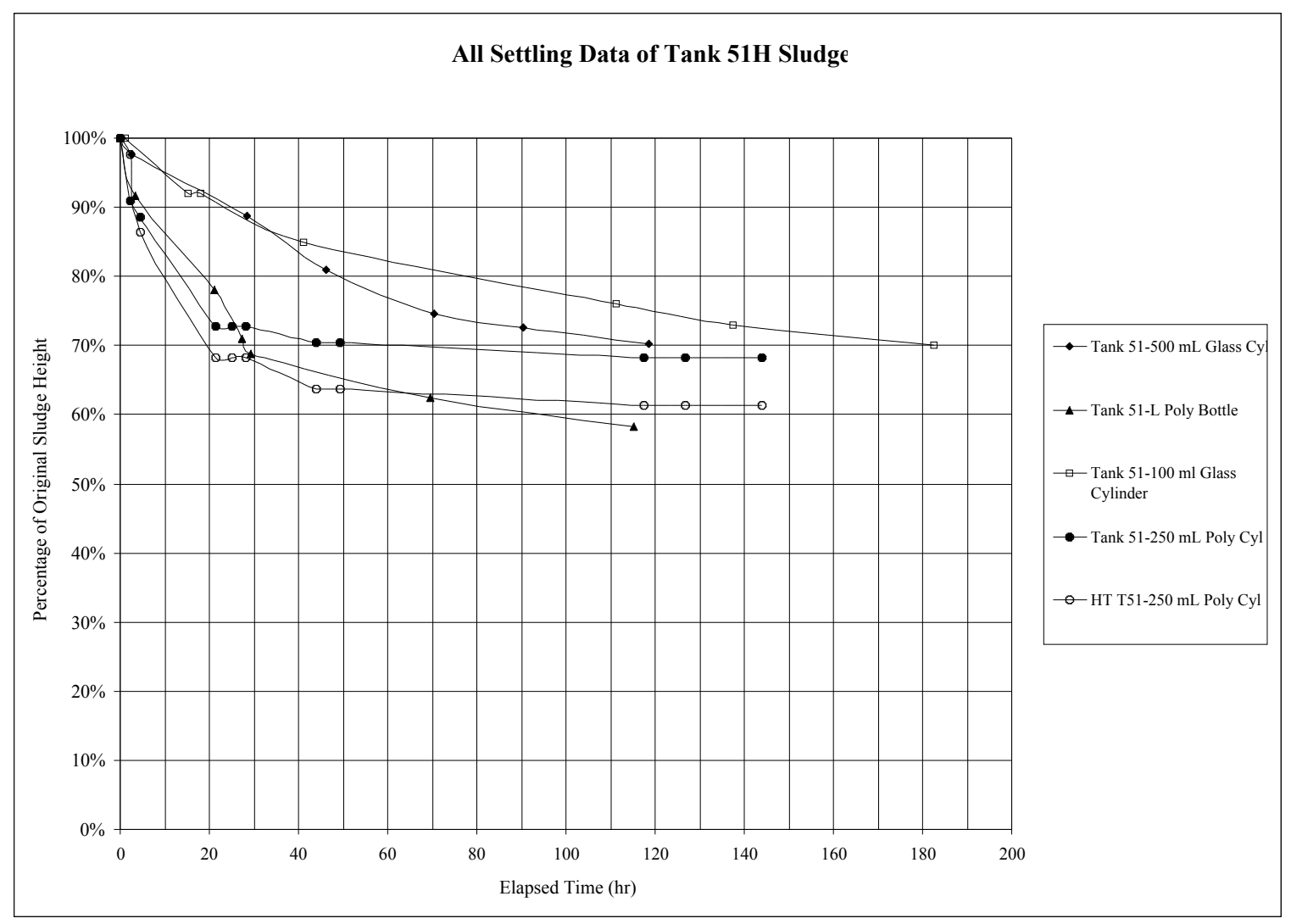

Figure 4-4. All Sludge Settling Data for Tank 51H Sludge 


\subsection{GENERAL DESCRIPTION OF ANALYTICAL PROCEDURES}

\subsection{INDUCTIVELY COUPLED PLASMA-ATOMIC EMISSION SPECTROSCOPY}

Samples were diluted as necessary to bring analytes within the instrument range. A scandium internal standard was added to all samples after dilution at a concentration of $2 \mathrm{mg} / \mathrm{L}$. Background and internal standard correction were applied to the results.

\subsection{ATOMIC ABSORPTION SPECTROSCOPY}

Mercury was analyzed by atomic absorption spectroscopy using the cold vapor (CV) technique. Samples were diluted as necessary to bring analytes within the instrument calibration range.

\subsection{ION CHROMATOGRAPHY}

For IC, samples were diluted with a carbonate/bicarbonate diluent as necessary to bring analytes to within instrument calibration for anion analysis.

\subsection{FREE HYDROXIDE AND CARBONATE TITRATIONS}

Free hydroxide was determined by incremental inflection point titration. The titration curves were examined to determine the free hydroxide concentration. Carbonate was determined by precipitation using saturated barium chloride. The precipitate was dissolved in acid and the carbonate concentration determined via back titration.

\subsection{INDUCTIVELY COUPLED PLASMA-MASS SPECTROMETRY}

Samples were diluted as necessary to bring analytes within the instrument range. The following describes the calculation of the analytes of interest from the mass values:

Tc-99 mass 99. Subject to interference when natural Ru is present in the sample. No natural Ru was present in the sample based on Ru mass ratios found.

$\mathrm{Ru} \quad$ mass 101,102 , and 104

$\mathrm{Rh}$ mass 103

Ag mass 107 and 109, Pd-107 subtracted from mass 107.

Pd mass 105 , other mass contributions $(106,107,108,110)$ calculated from fission yield.

Th $\quad$ mass 232

U-233 mass 233

U-234 mass 234

U-235 mass 235

U-236 mass 236

U-238 mass 238

Np-237 mass 237 
WSRC-TR-2005-00525, REV. 0

Pu-239 mass 239

$\mathrm{Pu}-240$ mass 240

$\mathrm{Am} / \mathrm{Pu}-241$ mass 241

$\mathrm{Pu}-242$ mass 242

Am-243 mass 243

Cm-244 mass 244

\subsection{GAMMA SPECTROMETRY}

An aliquot of each sample was analyzed by gamma spectroscopy analysis using a high purity germanium detector. Results are background subtracted.

\section{7 $\mathrm{SR}^{90}$ ANALYSIS}

An aliquot of each sample was analyzed for $\mathrm{Sr}^{90}$ using an Eichrom Sr-Spec based extraction procedure. A $\mathrm{Sr}^{90}$ spiked blank was analyzed with the sample batch to establish $\mathrm{Sr}^{90} / \mathrm{Y}^{90}$ counting efficiencies and $\mathrm{Sr}$ chemical recoveries. Aliquots of each sample's Sr extract were analyzed by neutron activation analysis to determine $\mathrm{Sr}$ carrier recoveries, the results of which were normalized to the results of the $\mathrm{Sr}^{90}$ spiked blank sample so each sample could be yielded by the recovery of its stable Sr carrier recovery. Once the extractions were complete, aliquots of the resultant $\mathrm{Sr}^{90} / \mathrm{Y}^{90}$ containing extracts mixed with liquid scintillation cocktail were counted in the ADS Radiochemistry Counting Facility. An instrument blank was counted first and was used to establish an instrument background that was subtracted from the count results for the samples.

\subsection{ALPHA SPECTROSCOPY FOR PLUTONIUM ISOTOPICS}

An aliquot of each sample was subjected to a TTA separation. An aliquot of the sample was initially spiked with a $\mathrm{Pu}^{238}$ tracer. A second aliquot of straight sample was analyzed along with the spiked sample. In addition, a third aliquot was used for determining the $\mathrm{Pu}^{241}$ concentration. All of the plutonium in the samples was reduced once using hydroxylamine. An anion complexing reagent (aluminum nitrate) was then added, and the solutions were oxidized with 4M sodium nitrite. The plutonium was then extracted from the matrix using a TTA solution. The TTA layer was mounted on a counting dish, the mount was then analyzed by alpha spectroscopy. A blank sample was run with the sample set.

The analysis results for the $\mathrm{Pu}^{239 / 240}$ alpha peak were yielded using the $\mathrm{Pu}^{238}$ recoveries from the $\mathrm{Pu}^{238}$ traced sample separation. The ratio of the $\mathrm{Pu}^{239 / 240}$ to the $\mathrm{Pu}^{238}$ in the sample was obtained from the alpha spectroscopy analysis of the non-spiked sample. That ratio was applied to the determined $\mathrm{Pu}^{239 / 240}$ value to determine the $\mathrm{Pu}^{238}$ activity in the sample.

The sample aliquot dedicated to the $\mathrm{Pu}^{241}$ analysis was added to liquid scintillation cocktail following the separation and analyzed for both $\mathrm{Pu}^{241}$ and gross $\mathrm{Pu}$-alpha constituents. The ratio of $\mathrm{Pu}^{241}$ to total $\mathrm{Pu}$ alpha was determined and applied to the results from the plates in order to determine a $\mathrm{Pu}^{241}$ concentration. 


\subsection{REFERENCES}

1. C.C. Herman, T.L. Fellinger, and S.L. Marra, SB4 - Tank 51 Settling Problems: Recommended Sampling, Characterization Plan, and Path Forward, SRNL-ITS2005-00125, June 9, 2005.

2. F.A. Washburn, Settling Tests in Support of Sludge Batch 4, HLW/DWPF/TTR-20050018, Rev. 0, June 29, 2005.

3. T. L. Fellinger, Investigation of Tank 51 Settling Issues in Support of Sludge Batch 4 (U), WSRC-RP-2005-01683, July 15, 2005.

4. T. L. Fellinger, Analytical Study Plan - Investigation of Tank 51 Settling Issues in Support of Sludge Batch 4 (U), SRNL-ITS-2005-00179, July 28, 2005.

5. C. J. Coleman, R. A. Dewberry, M. F. Bryant, J. J. Gemmill, SRL's Performance in Round Robin \#6 - Analysis of Simulated Defense Waste Glass (U), WSRC-TR-91187, Rev. 0, May 31, 1991.

6. T. L. Fellinger, M. S. Hay, Chemical and Radionuclide Analysis Results for the Tank $51 H$ Samples HTF-076 - HTF-081 (U), SRNL-ITS-2005-0268, November 9, 2005.

7. M. S. Hay, T. B. Edwards, Statistical Analysis of ESP Verification Test Samples, WSRC-RP-94-1224, November 4, 1994.

8. T. L. Fellinger, Corrosion Results for Tank 51 H Samples HTF-076 - HTF-081 (U), SRNL-ITS-2005-00203, August 19, 2005.

9. C. J. Bannochie, T. L. Fellinger, Tank 11 H Analytical Results as Input to DWPF Sludge Batch 4, WSRC-TR-2004-00473, September 2004.

10. T. Motyka, Technical Data Summary for In-Tank Sludge Processing, DPSTD-84100, April 1984.

11. M. S. Hay, N. E. Bibler, Characterization and Sludge Washing Studies of Tank 42H Sludge (U), WSRC-RP-94-730, July 27, 1994.

12. J. Gillam, Spreadsheet file ID: SB4_092005_baseline+.xls.

13. C. C. Herman, F. F. Fondeur, D. R. Click, M. E. Stone, Initial Results for SRNL Characterization and Settling Testing of Tank 51-Sludge Batch 4, SRNL-ITS-200500272, December 5, 2005. 